\title{
A hierarchy of topological tensor network states
}

\author{
Oliver Buerschaper ${ }^{1}$, Juan Martín Mombelli ${ }^{2}$, Matthias Christandl ${ }^{3,4}$, \\ Miguel Aguado ${ }^{1}$ \\ 1 Max-Planck-Institut für Quantenoptik, Hans-Kopfermann-Straße 1, 85748 Garching, Ger- \\ many \\ 2 Facultad de Matemática, Astronomía y Física, Universidad Nacional de Córdoba, Medina \\ Allende s/n, Ciudad Universitaria, 5000 Córdoba, Argentina \\ 3 Institute for Theoretical Physics, ETH Zurich, 8093 Zurich, Switzerland \\ 4 Fakultät für Physik, Ludwig-Maximilians-Universität München, Theresienstraße 37, 80333 \\ Munich, Germany
}

Received: October 23, 2018/ Accepted: date

\begin{abstract}
We present a hierarchy of quantum many-body states among which many examples of topological order can be identified by construction. We define these states in terms of a general, basis-independent framework of tensor networks based on the algebraic setting of finite-dimensional Hopf $C^{*}$-algebras. At the top of the hierarchy we identify ground states of new topological lattice models extending Kitaev's quantum double models [26. For these states we exhibit the mechanism responsible for their non-zero topological entanglement entropy by constructing a renormalization group flow. Furthermore it is shown that those states of the hierarchy associated with Kitaev's original quantum double models are related to each other by the condensation of topological charges. We conjecture that charge condensation is the physical mechanism underlying the hierarchy in general.
\end{abstract}

\section{Introduction}

1.1. Background. Quantum states of many-body systems, for instance lattice models of quantum spins, furnish a rich physical arena where simplified models of condensed matter phenomena such as high- $T_{c}$ superconductivity can be studied [2]. Such quantum systems are, however, difficult to solve numerically, since the dimension of the corresponding Hilbert space grows exponentially in the number of components.

Two promising developments tackling this difficulty have occurred in recent times. Firstly, the advent of experimental settings where lattice models can be implemented whose parameters can be controlled very accurately [6]. Secondly - and relevant for the present article - the understanding of the entanglement structure of models with local Hamiltonians. It is believed that ground states of such models generically obey an area law for the entanglement entropy [47,17. This has justified the development of a number of approaches where the physical 
states of the model can be described by so-called tensor networks whose set of parameters grows only polynomially in the number of components and which are therefore suitable for numerical simulations. Among these are the tensor product states (TPS) 38, also known as projected entangled-pair states (PEPS) 43, which generalize the structure of matrix product states (MPS) [19 to higher dimensions, and the multi-scale entanglement renormalisation ansatz (MERA) based on renormalisation group ideas 45, 46.

In 2003 Kitaev proposed topological quantum computation [26]: this is an approach to quantum computation which is based on quantum many-body systems exhibiting topological order, i.e. systems that are effectively described by a topological quantum field theory [49. Topological phases appear in condensed matter physics, for instance in the fractional quantum Hall effect [48; they can also be studied in the context of quantum field theories 3 . In Kitaev's proposal information is encoded in nontrivial loops of the underlying surface 15 and is processed by the creation, braiding and annihilation of topological charges 21, 22 . The simplest models proposed for this purpose are Kitaev's original quantum double models, which are based on discrete gauge theories [26, and Levin and Wen's string-net models [31. The latter are believed to describe all parity and time-reversal invariant topological phases on the lattice and are constructed from the idea that topological phases correspond to fixed points of renormalisation group procedures. In Kitaev's models, the space of superselection sectors is the set of irreducible representations of the quasitriangular Hopf algebra $\mathrm{D}(\mathbb{C} G)$, Drinfeld's quantum double [16] of the group algebra $\mathbb{C} G$ describing the gauge symmetry. We will therefore call these models $\mathrm{D}(\mathbb{C} G)$-models. The simplest such model is the well-known toric code which is based on $G=\mathbb{Z}_{2}$.

Recently, tensor networks for topological lattice models have been introduced and it is this development which forms the starting point for the current work. In 44, a PEPS ansatz is devised for a distinguished ground state of the toric code. Ground states of general quantum double models based on a group algebra can be described as MERA states [1, as can ground states of string-net models 30. Other tensor networks for string-nets are given in [11,23.

1.2. Aims. In the following we will outline the two main goals of this article: the extension of Kitaev's quantum double models and the derivation of tensor network representations for these generalized models.

The first aim of this article is to extend Kitaev's quantum double models 26 from the case of the group algebra of a finite group $G$ to a finite-dimensional Hopf algebra with certain properties, as anticipated by Kitaev in [26]. This sheds more light on the Hopf algebra point of view Kitaev outlined originally, in particular, it clarifies how the structure maps of the Hopf algebra enter in the definition of the model and how the quantum double of the Hopf algebra arises in the description of the model's excitations. It turns out that an involutory Hopf algebra (i.e. a Hopf algebra whose antipode map squares to the identity) with an additional *algebra structure is sufficient in order to define the generalized quantum double model properly. As we will show both requirements are satisfied if one chooses a finite-dimensional Hopf $C^{*}$-algebra (also known as a finite-dimensional Kac algebra). In order to illustrate the construction of our generalized model we give an example of a finite-dimensional Hopf $C^{*}$-algebra which is nontrivial in the sense that it is neither a group algebra nor the dual of a group algebra. 
This extends well-known instances of the family like the toric code for $G=$ $\mathbb{Z}_{2}$ or the model based on $G=S_{3}$ which is universal for topological quantum computation 35.

The second aim of the present article is to derive tensor network representations for the ground states of these models. As such representations allow to map out the phase diagram of the model and determine phase transitions between topologically and conventionally ordered phases it is crucial to understand the properties of tensor networks describing states of the model deep within the different phases. In order to derive these tensor network representations for quantum double model ground states we introduce a novel diagrammatic technique. It turns out that the tensor networks can be formulated basis-independently and that furthermore a single distinguished element of the Hopf $C^{*}$-algebra, namely its unique Haar integral, plays the key role in the construction. Other than that only the structure maps of the Hopf $C^{*}$-algebra are employed in the definition of the tensor network states.

In addition to the stated aims above we are able to extend these ground states to a hierarchy of tensor network states for each finite-dimensional Hopf $C^{*}$-algebra. This hierarchy of states is characterized by different values of the topological entanglement entropy $\gamma$ as defined in [27,32], and hence these states represent different instances of topological order, in other words different unitary modular tensor categories (UMTCs). The way this hierarchy depends on the Hopf subalgebras of the original Hopf $C^{*}$-algebra points towards condensation of topological charges 4,5. Furthermore we show how different isomorphism classes of finite-dimensional Hopf $C^{*}$-algebras exhibit different mechanisms for the non-zero $\gamma$. In particular, we explain how the boundary configurations of a region differ between models based on a group algebra, the dual of a group algebra and non-trivial finite-dimensional Hopf $C^{*}$-algebras.

1.3. $\mathrm{D}(\mathbb{C} G)$-models and their tensor network representations. We will now review the basics of Kitaev's quantum double model and its tensor network representations in a language appropriate for our later discussion.

The quantum double model presented in [26] is a quantum spin model defined on any finite oriented graph $\Gamma=(V, E, F)$ with vertices $V$, edges $E$ and faces $F$ which can be embedded in a two-dimensional surface. Throughout the article we will denote the set of all edges connected to a vertex $s \in V$ by $E(s)$ and similarly $E(p)$ will stand for the set of edges that form the boundary of the face $p \in F$. In order to construct the Hilbert space of the quantum double model one assigns to each oriented edge the local Hilbert space $\mathbb{C} G$ whose canonical basis is $\{|g\rangle \mid g \in G\}$. Reversing the orientation of an edge corresponds to the map $|g\rangle \mapsto\left|g^{-1}\right\rangle$. The Hamiltonian is assembled from two sets of operators that act on vertices or faces of $\Gamma$ respectively. At each vertex $s \in V$ one may orient the adjacent edges such that they point inwards. Then the vertex operator $A(s)$, which acts on $E(s)$, is given by

$$
A(s)=\frac{1}{|G|} \sum_{g \in G} L(g) \otimes \cdots \otimes L(g)
$$

where $L(g): \mathbb{C} G \rightarrow \mathbb{C} G,|h\rangle \mapsto|g h\rangle$ denotes the left regular representation of $G$. Hence the action of $A(s)$ is a simultaneous left multiplication at each incident 
edge averaged over the group $G$. In fact, it projects onto the trivial representation. Using the orientation reversal isomorphism one may orient the edges around a face $p \in F$ in counterclockwise fashion. One then defines the face operator $B(p)$ by its action on basis states $\left|g_{1}, \ldots, g_{r}\right\rangle$ :

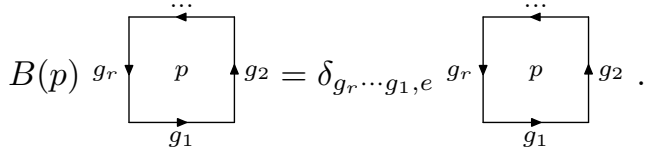

Again, this is a projector which projects onto configurations with a trivial product of group elements around the face. Finally, the Hamiltonian reads:

$$
\mathcal{H}=-\sum_{s \in V} A(s)-\sum_{p \in F} B(p) .
$$

This is a sum of mutually commuting projectors and therefore exactly solvable.

In order to find a tensor network representation for particular states occurring in the quantum double model one can start from its inherent (group) symmetries. We can assign a representation $\rho_{p}$ of $G$ to each face $p \in F$ and associate the tensor

$$
\underbrace{\rho_{p}}_{\rho_{p^{\prime}}} g=A\left(\rho_{p}, \rho_{p^{\prime}}\right)_{\alpha \beta \gamma \delta}^{g}:=\rho_{p}(g)_{\alpha \beta} \rho_{p^{\prime}}\left(g^{-1}\right)_{\gamma \delta}
$$

with each oriented edge. Here $\rho_{p}(g)_{\alpha \beta}$ denotes a matrix element of the representation $\rho_{p}$ and the red dot represents the physical index $g$ with its orientation inherited from the underlying graph edge. The Greek letters attached to black arrows are called virtual indices. The tensor for a reversed graph edge naturally reads

$$
\underbrace{\rho_{p}}_{\rho_{p^{\prime}}}=\underbrace{\alpha}_{\rho_{p^{\prime}}} \rho_{\delta}^{\rho_{p}}
$$

Reversing virtual arrows is defined by

$$
\underbrace{\rho_{p}}_{\rho_{p^{\prime}}} \alpha:=\underbrace{\alpha}_{\rho_{p^{\prime}}^{*}} g \text {. }
$$

where the dual representation $\rho^{*}$ of a representation $\rho$ is given by the matrix equation $\rho^{*}(g):=\rho^{T}\left(g^{-1}\right)$. Note that this is equivalent to a reflection of the tensor about its vertical axis:

$$
g=\underbrace{\rho_{p}}_{\rho_{p^{\prime}}}{ }_{\gamma}^{\alpha} g .
$$

The tensor network obtained by contracting all virtual indices around each face represents what we will call a group tensor network state in the following. 
For example, on a square lattice the tensor representing a vertex with a particular edge orientation is given by

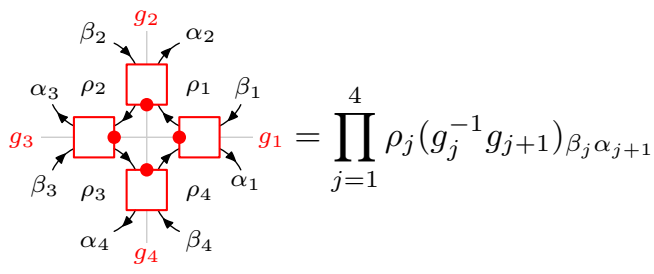

where the index $j$ labelling graph edges is assumed to be cyclic. Quite similarly, the tensor corresponding to a particular face reads

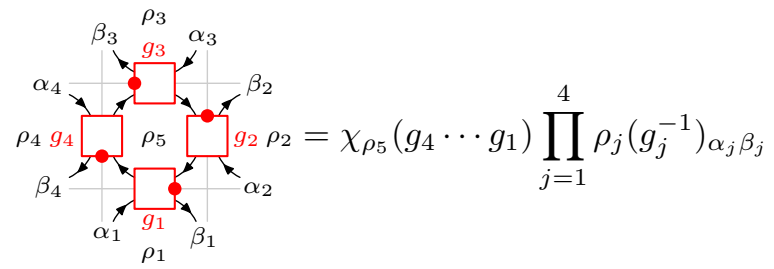

where $\chi_{\rho}$ is the character of the representation $\rho$.

In fact, both this vertex and face tensor are partially contracted tensor networks and represent linear maps from the uncontracted virtual indices to the physical ones. So a piece of a tensor network with open indices can be regarded as a PEPS projection map [42,36. If $V_{p}$ is the vector space associated with the representation $\rho_{p}$ then this linear map $P: V_{p} \otimes V_{p} \otimes V_{p^{\prime}} \otimes V_{p^{\prime}} \rightarrow \mathbb{C} G$ is given by

$$
P=\sum_{g, \alpha, \ldots, \delta \in G} A\left(\rho_{p}, \rho_{p^{\prime}}\right)_{\alpha \beta \gamma \delta}^{g}|g\rangle\langle\alpha, \beta, \gamma, \delta|
$$

for a single edge tensor $A\left(\rho_{p}, \rho_{p^{\prime}}\right)$.

On the contrary, a fully contracted tensor network is a complex number which equals the inner product between a particular basis state and the group tensor network state. If viewed as a linear map from basis configurations to amplitudes we will call it a tensor trace.

Clearly, a group tensor network state only depends on the isomorphism class of the representations $\rho_{p}$ assigned to the faces. In general, there is vastly more gauge freedom for a tensor network to represent the same physical state, however, this does not necessarily respect the $G$-action.

If we choose all representations $\rho_{p}$ to coincide with the left regular one the resulting group tensor network state will be a ground state of the $\mathrm{D}(\mathbb{C} G)$-model. Since its Hamiltonian is frustration free (i.e. a sum of mutually commuting terms) this can easily be shown by considering independently the action of $A(s)$ and $B(p)$ on the partially contracted tensor networks in (8) and $(9)$.

As an example, a ground state of the toric code is given by the group tensor network for the group $\mathbb{Z}_{2}=\{e, a\}$ with all faces carrying the regular representation. In that case the PEPS projection map reads $P=|e\rangle\left\langle\phi^{+}\right|\left\langle\phi^{+}\right|+$ 
$|a\rangle\left\langle\psi^{+}\right|\left\langle\psi^{+}\right|$with the Bell states

$$
\begin{aligned}
& \left|\phi^{+}\right\rangle=\sum_{\alpha, \beta \in \mathbb{Z}_{2}} L(e)_{\alpha \beta}|\alpha, \beta\rangle=|e, e\rangle+|a, a\rangle, \\
& \left|\psi^{+}\right\rangle=\sum_{\alpha, \beta \in \mathbb{Z}_{2}} L(a)_{\alpha \beta}|\alpha, \beta\rangle=|e, a\rangle+|a, e\rangle .
\end{aligned}
$$

We would like to remark that this projection map coincides (up to a trivial isomorphism) with the one given in [44.

Later in the article we will turn to the physical significance of group tensor network states arising from representations of $G$ other than the regular one.

In order to build some more intuition for the main part of this article we briefly review the structure of a local tensor in a group tensor network state. It is easily seen that the tensor in (4) factorizes with respect to partitioning the virtual indices into the sets $\{\alpha, \beta\}$ and $\{\gamma, \delta\}$. This implies that the PEPS projection map $(10)$ can be rewritten as:

$$
P=\sum_{g \in G}|g\rangle\left\langle\phi_{p}(g)\right|\left\langle\phi_{p^{\prime}}\left(g^{-1}\right)\right|
$$

where a state associated to the virtual indices in face $p$ is given by:

$$
\left|\phi_{p}(g)\right\rangle=\sum_{\alpha, \beta \in G} \overline{\rho_{p}(g)_{\alpha \beta}}|\alpha, \beta\rangle .
$$

This means that one can carry out the contraction of the tensor network in two steps. First one contracts each virtual loop separately and then one needs to glue these pieces together. In a group tensor network this glueing process is simply given by

$$
|g\rangle \otimes\left|g^{-1}\right\rangle \mapsto|g\rangle .
$$

It turns out that this step becomes rather nontrivial once we generalize the quantum double model to finite-dimensional Hopf $C^{*}$-algebras. In fact, we anticipate that this glueing step is one of the crucial ingredients for deriving the hierarchy of tensor network states in this article.

1.4. Outline. The article is structured as follows. In Section 2, we will provide a guide to the language of Hopf algebras, introducing the necessary notation needed in order to extend the quantum double models based on finite groups to Hopf algebras. Characteristic examples of Hopf algrebras relevant for this work are supplied, too. In Section 3 we then generalize Kitaev's quantum double construction from group algebras to Hopf algebras, thereby introducing the physical model which stands in the centre of this work. In Section 4, we will solve this model by providing a tensor network representation of its ground state. This representation only involves the canonical structures associated to the underlying Hopf algebra and leads us to propose a novel hierarchy of tensor network states based on Hopf subalgebras. In Section 5 we calculate both the entanglement entropy and the topological entanglement entropy for distinguished states in the hierarchy. In section 6 we provide concluding remarks and an outlook on future work. 


\section{The language of Hopf algebras}

2.1. Hopf algebras. If one sets out to find an algebraic structure for symmetries of linear spaces with tensor product structure, such as encountered in many-body quantum physics, one is naturally led to the concept of a Hopf algebra. This is because the axioms of a Hopf algebra directly allow for duals and tensor products of representations. As general reference for this section we recommend 25.

First and foremost a Hopf algebra $H$ is a vector space (over some field $k$ which we will take to be $\mathbb{C}$ ) equipped with some additional structure. On the one hand there is an associative multiplication $\mu$ of vectors which is linear in each argument, hence one may define it as the linear map $\mu: H \otimes H \rightarrow H$ with

$$
\mu(x \otimes y)=x y
$$

such that

$$
(x y) z=x(y z) .
$$

This multiplication is accompanied by a unit $\eta$ which can be defined as the linear injection of scalars into $H$, hence $\eta: k \rightarrow H$ where

$$
\eta(\lambda)=\lambda 1_{H}
$$

The element $1_{H} \in H$ is a left and right unit for the multiplication $\mu$. At this stage $H$ has the structure of an algebra. The multiplication encodes the composition of symmetry transformations in a representation space.

On the other hand there is a dual notion to multiplication. This dual linear map is the comultiplication $\Delta: H \rightarrow H \otimes H$ and is usually written in Sweedler notation 11 as

$$
\Delta(x)=\sum_{(x)} x^{\prime} \otimes x^{\prime \prime}=\sum_{(x)} x^{(1)} \otimes x^{(2)} .
$$

This serves as a shorthand for sums of the form $\sum_{i} x_{i}^{(1)} \otimes x_{i}^{(2)}$. It is required to be coassociative:

$$
(\Delta \otimes \mathrm{id}) \circ \Delta=(\mathrm{id} \otimes \Delta) \circ \Delta .
$$

Elements which satisfy $\Delta(x)=x \otimes x$ are called grouplike and collected in the set $\mathcal{G}(H)$. This comultiplication has a companion called the counit $\epsilon: H \rightarrow k$ which is required to satisfy the axiom:

$$
\sum_{(x)} \epsilon\left(x^{\prime}\right) x^{\prime \prime}=\sum_{(x)} x^{\prime} \epsilon\left(x^{\prime \prime}\right)=x .
$$

In other words, it neutralizes the comultiplication. With this structure alone, the vector space $H$ is called a coalgebra. The comultiplication encodes how symmetry transformations act on tensor products of representation spaces. The counit provides the appropriate notion of a trivial representation, or invariance under symmetry transformations.

\footnotetext{
1 If there are more than three tensor factors in iterated coproducts such as $\Delta^{(3)}(x)=$ $\sum_{(x)} x^{(1)} \otimes x^{(2)} \otimes x^{(3)} \otimes x^{(4)}$ we will consistently use superscripts instead of primes.
} 
If both the algebra and coalgebra structure are compatible then $H$ is called a bialgebra. The compatibility axioms read:

$$
\begin{aligned}
\Delta(x y) & =\Delta(x) \Delta(y), \\
\Delta\left(1_{H}\right) & =1_{H} \otimes 1_{H}, \\
\epsilon(x y) & =\epsilon(x) \epsilon(y), \\
\epsilon\left(1_{H}\right) & =1_{k} .
\end{aligned}
$$

Finally, if there is a linear map $S: H \rightarrow H$ satisfying the axiom

$$
\sum_{(x)} x^{\prime} S\left(x^{\prime \prime}\right)=\epsilon(x) 1_{H}=\sum_{(x)} S\left(x^{\prime}\right) x^{\prime \prime}
$$

then $H=(H ; \mu, \eta ; \Delta, \epsilon ; S)$ is called a Hopf algebra. The map $S$ is called the antipode and has the following properties:

$$
\begin{aligned}
S(x y) & =S(y) S(x), \\
S\left(1_{H}\right) & =1_{H}, \\
\sum_{(S(x))} S(x)^{\prime} \otimes S(x)^{\prime \prime} & =\sum_{(x)} S\left(x^{\prime \prime}\right) \otimes S\left(x^{\prime}\right), \\
\epsilon(S(x)) & =\epsilon(x) .
\end{aligned}
$$

In any finite-dimensional Hopf algebra the order of the antipode $S$ is finite [40, hence $S$ is an invertible map. We will always make this assumption in the sequel. The antipode is used to define dual (or conjugate) representations.

We may also define opposite multiplication $\mu^{\mathrm{op}}$ and comultiplication $\Delta^{\mathrm{cop}}$ by

$$
\begin{aligned}
\mu^{\mathrm{op}}(x \otimes y) & =y x, \\
\Delta^{\mathrm{cop}}(x) & =\sum_{(x)} x^{\prime \prime} \otimes x^{\prime}
\end{aligned}
$$

relative to the multiplication (16) and comultiplication (19). Then the sets

$$
\begin{aligned}
Z(H) & =\left\{x \in H \mid \forall y \in H: \mu^{\mathrm{op}}(x \otimes y)=\mu(x \otimes y)\right\} \\
\operatorname{Cocom}(H) & =\left\{x \in H \mid \Delta^{\mathrm{cop}}(x)=\Delta(x)\right\}
\end{aligned}
$$

are called the centre of $H$ and the cocommutative elements of $H$ respectively. If $Z(H)=H$ then $H$ is a commutative Hopf algebra, in the case $\operatorname{Cocom}(H)=$ $H$ it is a cocommutative Hopf algebra. Furthermore it turns out that $H^{\mathrm{op}}=$ $\left(H ; \mu^{\mathrm{op}}, \eta ; \Delta, \epsilon ; S^{-1}\right)$ is again a Hopf algebra, called the opposite Hopf algebra of $H$.

One can now show that for a given Hopf algebra $H=(H ; \mu, \eta ; \Delta, \epsilon ; S)$ with underlying vector space $H$ the dual vector space $H^{*}$ has again the structure of a Hopf algebra. More precisely,

$$
H^{*}=\left(H^{*} ; \Delta^{T}, \epsilon^{T} ; \mu^{T}, \eta^{T} ; S^{T}\right)
$$


with the structure map $\AA^{2}$ as indicated is called the dual Hopf algebra of $H$. By the same token, the opposite Hopf algebra $H^{\mathrm{op}}$ has the natural dual

$$
X=\left(H^{\mathrm{op}}\right)^{*}=\left(H^{*} ; \Delta^{T}, \epsilon^{T} ;\left(\mu^{\mathrm{op}}\right)^{T}, \eta^{T} ;\left(S^{-1}\right)^{T}\right) .
$$

Finally, there is the notion of a (two-sided) integral $\Lambda$ in a Hopf algebra $H$. Such an element is defined via

$$
x \Lambda=\Lambda x=\epsilon(x) \Lambda
$$

for all $x \in H$. In view of the role of the counit as a trivial representation, integrals are thus invariant elements under multiplication. The dual definition of an integral $\Gamma \in H^{*}$ can be phrased as

$$
\sum_{(x)} x^{\prime} \Gamma\left(x^{\prime \prime}\right)=\sum_{(x)} \Gamma\left(x^{\prime}\right) x^{\prime \prime}=\Gamma(x) 1_{H}
$$

for all $x \in H$. This turns out to be equivalent to (37) once applied to $H^{*}$.

Particularly important is the notion of a Haar integral. This is the normalized version of an integral which is guaranteed to exist uniquely for finite-dimensional Hopf $C^{*}$-algebras. Those are the Hopf algebras we will be occupied with mostly in the discussion of the physical model. We will supply a precise definition of Haar integrals in Section 3.2 .

2.2. Quantum doubles as bicrossed products. In a general Hopf algebra $H$ nothing can be said about how $\Delta$ and $\Delta^{\text {cop }}$ are related to each other. In other words, $H$ can be non-cocommutative in the most unpleasant way. Therefore we seek Hopf algebras which are almost cocommutative ${ }^{3}$ in a certain sense: they are equipped with a so-called quasitriangular structure which controls the extent to which the comultiplication fails to be cocommutative. In [16] Drinfeld gave his celebrated quantum double construction which produces such a quasitriangular Hopf algebra from a given Hopf algebra.

At the heart of his construction lies the idea of a bicrossed product. For example, in the case of the semidirect product of two groups one has an action of one group on the other and this action is used to define the multiplication in the product group. Similarly, one may define a bicrossed product of groups where there is an additional backaction of the second group on the first one. It is this concept that can be generalized to Hopf algebras and will yield the quantum double.

So given a Hopf algebra $H$ with invertible antipode $S$ (in particular, any finite-dimensional Hopf algebra) one can construct a new Hopf algebra $\mathrm{D}(H)=$ $X \bowtie H$, the quantum double of $H$, as the bicrossed product of $X=\left(H^{\mathrm{op}}\right)^{*}$ and $H$. This means that as a vector space $\mathrm{D}(H)$ simply equals $H^{*} \otimes H$. In order

\footnotetext{
2 For a linear map $F: U \rightarrow V$ the transpose map $F^{T}: V^{*} \rightarrow U^{*}$ is defined as usual by $\left\langle F^{T}(\alpha), x\right\rangle:=\langle\alpha, F(x)\rangle$ for all $\alpha \in V^{*}$ and $x \in U$.

3 In physical applications this is related to the exchange statistics of particles in two dimensions.
} 
to define its multiplication we need an action of $H$ on $X$ and another one vice versa. This bicrossed structure is given by the actions

$$
\begin{array}{ll}
\triangleright: H \times X \rightarrow X, & a \triangleright f=\sum_{(a)} f\left(S^{-1}\left(a^{\prime \prime}\right) ? a^{\prime}\right), \\
\triangleleft: H \times X \rightarrow H, & a \triangleleft f=\sum_{(a)} f\left(S^{-1}\left(a^{\prime \prime \prime}\right) a^{\prime}\right) a^{\prime \prime},
\end{array}
$$

where the question mark denotes the argument of the function $a \triangleright f$, i.e. in this particular instance

$$
(a \triangleright f)(x)=\sum_{(a)} f\left(S^{-1}\left(a^{\prime \prime}\right) x a^{\prime}\right)
$$

holds for any $x \in H$. We will use this notation frequently later on.

One can then show that

$$
(f \otimes a)(g \otimes b)=\sum_{(a)} f g\left(S^{-1}\left(a^{\prime \prime \prime}\right) ? a^{\prime}\right) \otimes a^{\prime \prime} b,
$$

together with the canonical comultiplication on the tensor product of Hopf algebras, defines a valid Hopf algebra structure on the vector space $\mathrm{D}(H)$. Since both the embeddings $i_{X}: X \rightarrow \mathrm{D}(H), f \mapsto f \otimes 1_{H}$ and $i_{H}: H \rightarrow \mathrm{D}(H), a \mapsto 1_{X} \otimes a$ are algebra morphisms this multiplication formula is actually already determined by the so-called straightening formula ${ }^{4}$

$$
\text { af }:=\left(1_{X} \otimes a\right)\left(f \otimes 1_{H}\right)=\sum_{(a)} f\left(S^{-1}\left(a^{\prime \prime \prime}\right) ? a^{\prime}\right) a^{\prime \prime} .
$$

It should be noted that both underlying actions $(39)$ and $(40)$ are derived from the adjoint representation which for $a, x \in H$ is defined by

$$
\operatorname{ad}(a)(x)=\sum_{(a)} a^{\prime} x S\left(a^{\prime \prime}\right) .
$$

2.3. Hopf $*$-algebras. If $k=\mathbb{C}$ then a Hopf algebra $H$ may sometimes be equipped with a so-called $*$-structure that will become most important for physical applications. Namely, it will allow us to define Hilbert spaces and unitarity. For a general reference on Hopf $*$-algebras see [28.

Firstly, a conjugate-linear map $*: H \rightarrow H$ which satisfies

$$
\begin{aligned}
& \left(x^{*}\right)^{*}=x \\
& (x y)^{*}=y^{*} x^{*}
\end{aligned}
$$

is called an involution and turns $H$ into a $*$-algebra. It follows naturally that

$$
1_{H}^{*}=1_{H} .
$$

4 This should be contrasted with the canonical (uncrossed) multiplication in the Hopf algebra $X \otimes H$. In this case we simply have $a f=f a$ for any $f \in X$ and $a \in H$ since multiplication is defined componentwise. 
If an involution $*$ is compatible with comultiplication

$$
\Delta\left(x^{*}\right)=\Delta(x)^{*}
$$

then $H$ is called a *-coalgebra. Here the involution of $H \otimes H$ is defined by $(x \otimes y)^{*}=x^{*} \otimes y^{*}$. In a $*$-coalgebra one always has

$$
\epsilon\left(x^{*}\right)=\overline{\epsilon(x)}
$$

where the bar denotes complex conjugation.

A bialgebra $H$ with an involution for which it is both a *-algebra and a *-coalgebra is called a $*$-bialgebra.

Interestingly, if a Hopf algebra $H$ also has the structure of a $*$-bialgebra then the interplay between antipode and involution is already determined:

$$
S\left(S\left(x^{*}\right)^{*}\right)=x .
$$

Consequently, $H$ is called a Hopf $*$-algebra. Note that its antipode is always invertible (even if $H$ is not finite-dimensional).

Finally, one can show that the dual of a Hopf $*$-algebra $H$ is again a Hopf *-algebra with the involution given by

$$
f^{*}(x)=\overline{f\left(S(x)^{*}\right)} .
$$

2.4. Trivial Hopf algebras and their quantum doubles. Here we briefly discuss some cases of how Hopf algebras can arise from groups. In particular we focus on how a group itself can be directly understood in the language of Hopf algebras. This will be the key for relating Kitaev's original construction to our generalization.

2.4.1. Trivial Hopf algebras. A finite-dimensional Hopf algebra is said to be trivial if it is a group algebra or the dual of a group algebra for some finite group $G$. Let $\{g \mid g \in G\}$ be a basis of the group algebra $\mathbb{C} G$ with multiplication

$$
\mu(g \otimes h)=g h
$$

and unit $\eta\left(1_{\mathbb{C}}\right)=e$. Its comultiplication and counit are given by

$$
\Delta(g)=g \otimes g, \quad \epsilon(g)=1_{\mathbb{C}}
$$

and extended by linearity. Similarly, the antipode map is defined by

$$
S(g)=g^{-1} .
$$

It is easy to see that every group algebra $\mathbb{C} G$ is cocommutative, but in general non-commutative. Furthermore, one may endow $\mathbb{C} G$ with the involution map

$$
g^{*}=g^{-1}
$$

which is extended by conjugate linearity. This turns $\mathbb{C} G$ into a Hopf $C^{*}$-algebra. Its Haar integral reads

$$
h=\frac{1}{|G|} \sum_{g \in G} g .
$$


The dual $(\mathbb{C} G)^{*}$ of a group algebra coincides with the space $\mathbb{C}^{G}$ of linear functions from the group to the field of complex numbers. For the dual basis $\left\{\delta_{g} \mid\right.$ $g \in G\}$ multiplication and unit are defined by

$$
\delta_{g} \delta_{h}=\delta_{g, h} \delta_{g}, \quad \eta\left(1_{\mathbb{C}}\right)=\sum_{g \in G} \delta_{g}=1
$$

where $\delta_{g, h}$ denotes the Kronecker delta. The comultiplication, counit and antipode maps are given by

$$
\Delta\left(\delta_{g}\right)=\sum_{u v=g} \delta_{u} \otimes \delta_{v}, \quad \epsilon\left(\delta_{g}\right)=\delta_{g}(e)=\delta_{g, e}, \quad S\left(\delta_{g}\right)=\delta_{g^{-1}}
$$

for each $g \in G$. One may check that every function algebra $\mathbb{C}^{G}$ is commutative, but in general non-cocommutative. When contrasted with the corresponding group algebra, this is not too surprising: since $\mathbb{C} G$ and $\mathbb{C}^{G}$ are dual to each other, one obtains the multiplication of one algebra from the comultiplication of the other one and vice versa. Again, $\mathbb{C}^{G}$ is a Hopf $C^{*}$-algebra via

$$
\delta_{g}^{*}=\delta_{g}
$$

and conjugate-linear extension 5 . Finally,

$$
\phi=\delta_{e}
$$

is the Haar integral of $\mathbb{C}^{G}$.

2.4.2. Quantum doubles of trivial Hopf algebras. As a vector space the quantum double of a group algebra is nothing but $\mathrm{D}(\mathbb{C} G)=\mathbb{C}^{G} \otimes \mathbb{C} G$ with basis $\left\{\delta_{x} \otimes g \mid\right.$ $(x, g) \in G \times G\}$. The fact that the quantum double is a crossed product is reflected in the multiplication map

$$
\left(\delta_{x} \otimes g\right)\left(\delta_{y} \otimes h\right)=\delta_{x, g y g^{-1}} \delta_{x} \otimes g h
$$

where the algebra acts on itself by conjugation. Actually, this is fully determined by the straightening formula

$$
g \delta_{x}:=(1 \otimes g)\left(\delta_{x} \otimes e\right)=\delta_{g x g^{-1}} \otimes g .
$$

(and the embeddings $\delta_{x} \mapsto \delta_{x} \otimes e$ and $g \mapsto 1 \otimes g$ being algebra morphisms) Here $g \delta_{x}$ is a frequently used shorthand notation and should be clear from context whenever it appears. The unit of $\mathrm{D}(\mathbb{C} G)$ is $1 \otimes e$. Comultiplication and counit are given by

$$
\begin{aligned}
\Delta\left(\delta_{x} \otimes g\right) & =\sum_{u v=x}\left(\delta_{v} \otimes g\right) \otimes\left(\delta_{u} \otimes g\right), \\
\epsilon\left(\delta_{x} \otimes g\right) & =\epsilon(g) \delta_{x}(e)=\delta_{x, e}
\end{aligned}
$$

5 If $G$ is an Abelian group then $\delta_{g}^{\star}=\delta_{g^{-1}}$ defines another Hopf $*$-structure for the standard convention. In fact, for $G$ non-Abelian the choice of $*$ or $\star$ corresponds to a choice of either the standard convention or the convention $(a \otimes b)^{\star}=b^{\star} \otimes a^{\star}$ for the action on tensor products. 
on the basis elements. Note that the comultiplication in $\mathrm{D}(\mathbb{C} G)$ is derived from the comultiplication in $\mathbb{C} G$ and $\left(\mathbb{C}^{G}\right)^{\text {cop }}=\left((\mathbb{C} G)^{\text {op }}\right)^{*}$ rather than from $\mathbb{C}^{G}$ itself! This is because as an algebra the quantum double is the bicrossed product $\mathrm{D}(\mathbb{C} G)=\left((\mathbb{C} G)^{\mathrm{op}}\right)^{*} \bowtie \mathbb{C} G$.

The antipode map is

$$
S\left(\delta_{x} \otimes g\right)=\delta_{g^{-1} x^{-1} g} \otimes g^{-1} .
$$

2.4.3. Hopf subalgebras of trivial Hopf algebras. The Hopf subalgebras of a Hopf algebra will be important for the construction of our hierarchy of Hopf tensor network states.

If $H=\mathbb{C} G$, all the Hopf subalgebras $A$ and $B$ can be characterized easily [4,5]. One finds that the Hopf subalgebras of $\mathbb{C} G$ are precisely the group algebras $\mathbb{C} K$ for $K \subset G$ a subgroup. Furthermore the Hopf subalgebras of $\mathbb{C}^{G}$ are exactly given by $\mathbb{C}^{G / N}$ with $N \triangleleft G$ a normal subgroup. It is also not difficult to find the Haar integrals of these Hopf subalgebras. In the first case one has

$$
h_{K}=\frac{1}{|K|} \sum_{g \in K} g .
$$

For the second case note that $\mathbb{C}^{G / N}$ can be identified with the functions in $\mathbb{C}^{G}$ which are constant on cosets of $N$. Now consider the character $\chi_{N}$ of $G$ that factors through $N$ to the regular character of $G / N$. It is easy to see that when multiplied with the above functions $\chi_{N}$ acts as a two-sided integral. Hence taking normalization into account, one may convince oneself that $|G / N|^{-1} \chi_{N}$ is precisely the Haar integral of $\mathbb{C}^{G / N}$. By abuse of notation we may write it as:

$$
\phi_{N}=\sum_{n \in N} \delta_{n}
$$

2.5. An example of a nontrivial finite-dimensional Hopf $C^{*}$-algebra. We are primarily interested in finite-dimensional nontrivial Hopf $C^{*}$-algebras, i.e. finitedimensional nontrivial Hopf algebras over $\mathbb{C}$ with a compatible involution. In order to find an example of such a Hopf algebra it is enough to investigate semisimple Hopf algebras in low dimensions and check whether these support a compatible $*$-structure. It turns out that any semisimple Hopf algebra $H$ of dimension $p$ or $p q$ with prime numbers $p<q$ is trivial [18. The same applies to dimensions $p^{2}$ [34]. Hence one may expect nontrivial semisimple Hopf algebras only for $\operatorname{dim} H \geq 8$.

2.5.1. The Hopf algebra $H_{8}$. In fact, we need to look no further than $\operatorname{dim} H=8$ for an example. At this dimension there exists a unique nontrivial semisimple Hopf algebra (up to isomorphism) which is commonly denoted by $H_{8}$ and is due to Kac and Paljutkin 24. Interestingly, it can also be endowed with a compatible involution which turns $H_{8}$ into a Hopf $C^{*}$-algebra.

Following 33 this algebra can be presented by generators $x, y, z$ and the relations

$$
\begin{gathered}
x^{2}=y^{2}=1, \quad z^{2}=\frac{1}{2}(1+x+y-x y), \\
x y=y x, \quad z x=y z, \quad z y=x z .
\end{gathered}
$$


It is now easy to see that $\mathcal{B}=\{1, x, y, x y, z, z x, z y, z x y\}$ is a basis of $H_{8}$. We will denote the corresponding dual basis by $\left\{\delta_{i} \mid i \in \mathcal{B}\right\}$. The coalgebra structure is determined by

$$
\begin{aligned}
& \Delta(x)=x \otimes x, \quad \Delta(y)=y \otimes y, \\
& \Delta(z)=\frac{1}{2}(1 \otimes 1+y \otimes 1+1 \otimes x-y \otimes x)(z \otimes z), \\
& \epsilon(x)=\epsilon(y)=\epsilon(z)=1,
\end{aligned}
$$

and from this it is evident that $\mathcal{G}\left(H_{8}\right)=\{1, x, y, x y\} \simeq \mathbb{Z}_{2} \times \mathbb{Z}_{2}$. The antipode reads

$$
S(x)=x, \quad S(y)=y, \quad S(z)=z .
$$

Finally, the involution is given by

$$
x^{*}=x, \quad y^{*}=y, \quad z^{*}=z^{-1}=\frac{1}{2}(z+z x+z y-z x y)=z^{3} .
$$

This shows in particular that antipode and involution act differently on the generators of $H_{8}$.

The Haar integrals $h \in H_{8}$ and $\phi \in H_{8}^{*}$ are given by:

$$
\begin{aligned}
& h=\frac{1}{8}(1+x+y+x y+z+z x+z y+z x y), \\
& \phi=\delta_{1} .
\end{aligned}
$$

Remarkably they look like the Haar integrals of a group algebra and its dual! The dual Haar integral $\phi$ and the complex involution automatically yield a Hermitian inner product on $H_{8}$ as discussed in Section 3.2. Explicitly, we have for all $a, b \in H_{8}$

$$
(a, b)=\delta_{1}\left(a^{*} b\right)
$$

It follows that $\mathcal{B}$ is an orthonormal basis of $H_{8}$.

2.5.2. Hopf subalgebras of $H_{8}$ and its dual. The Hopf subalgebras of $H=H_{8}$ $\operatorname{read}\{1\},\{1, x\} \simeq \mathbb{C}_{2},\{1, y\} \simeq \mathbb{C}_{2},\{1, x y\} \simeq \mathbb{C}_{2},\{1, x, y, x y\} \simeq \mathbb{C}\left(\mathbb{Z}_{2} \times \mathbb{Z}_{2}\right)$ and $H_{8}$. This is because Hopf subalgebras of a Hopf algebra $H$ correspond bijectively to sets of irreducible representations of $H^{*}$ which close under fusion and dualisation [4,5. Now $H_{8}^{*}$ has four one-dimensional irreducible representations $Q_{1}, Q_{x}, Q_{y}$ and $Q_{x y}$ derived from $\mathcal{G}\left(H_{8}\right)$ and the two-dimensional representation given by

$$
Q_{2}=\frac{1}{2}\left(\begin{array}{cc}
z+z x & z-z x \\
z y-z x y & z y+z x y
\end{array}\right)
$$

From the fusion rules

$$
\begin{aligned}
Q_{g} \times Q_{g^{\prime}} & =Q_{g g^{\prime}} \\
Q_{g} \times Q_{2} & =Q_{2} \\
Q_{2} \times Q_{2} & =Q_{1}+Q_{x}+Q_{y}+Q_{x y}
\end{aligned}
$$

for all $g, g^{\prime} \in \mathcal{G}\left(H_{8}\right)$ one concludes that precisely the sets $\left\{Q_{1}\right\},\left\{Q_{1}, Q_{x}\right\}$, $\left\{Q_{1}, Q_{y}\right\},\left\{Q_{1}, Q_{x y}\right\},\left\{Q_{1}, Q_{x}, Q_{y}, Q_{x y}\right\}$ and $\left\{Q_{1}, Q_{x}, Q_{y}, Q_{x y}, Q_{2}\right\}$ close under 
fusion (and dualization) and therefore are the ones that correspond bijectively to Hopf subalgebras of $H_{8}$.

The Haar integrals of the Hopf subalgebras read

$$
\begin{aligned}
h_{\{1\}} & =1, \\
h_{\langle x\rangle} & =\frac{1}{2}(1+x), \\
h_{\langle y\rangle} & =\frac{1}{2}(1+y), \\
h_{\langle x y\rangle} & =\frac{1}{2}(1+x y), \\
h_{\langle x, y\rangle} & =\frac{1}{4}(1+x+y+x y), \\
h & =\frac{1}{8}(1+x+y+x y+z+z x+z y+z x y) .
\end{aligned}
$$

Since $H_{8}^{*} \simeq H_{8}$ as Hopf algebras the Hopf subalgebras $B \subset H_{8}^{*}$ are given as the images of the Hopf subalgebras of $H_{8}$ under the isomorphism. A particular such Hopf isomorphism can be obtained as follows. From the dual basis $\left\{\delta_{a}\right\}$ of the basis $\mathcal{B} \subset H_{8}$ define another basis $\left\{f_{a}\right\} \subset H_{8}^{*}$ via

$$
\begin{aligned}
f_{1} & :=\delta_{1}+\delta_{x}+\delta_{y}+\delta_{x y}+\delta_{z}+\delta_{z x}+\delta_{z y}+\delta_{z x y}, \\
f_{x} & :=\delta_{1}+\delta_{x}+\delta_{y}+\delta_{x y}-\delta_{z}-\delta_{z x}-\delta_{z y}-\delta_{z x y}, \\
f_{y} & :=\delta_{1}-\delta_{x}-\delta_{y}+\delta_{x y}+i \delta_{z}-i \delta_{z x}-i \delta_{z y}+i \delta_{z x y}, \\
f_{x y} & :=\delta_{1}-\delta_{x}-\delta_{y}+\delta_{x y}-i \delta_{z}+i \delta_{z x}+i \delta_{z y}-i \delta_{z x y}, \\
f_{z} & :=\delta_{1}+\delta_{x}-\delta_{y}-\delta_{x y}+\delta_{z}+\delta_{z x}-\delta_{z y}-\delta_{z x y} \\
f_{z x} & :=\delta_{1}-\delta_{x}+\delta_{y}-\delta_{x y}+\delta_{z}-\delta_{z x}+\delta_{z y}-\delta_{z x y}, \\
f_{z y} & :=\delta_{1}+\delta_{x}-\delta_{y}-\delta_{x y}-\delta_{z}-\delta_{z x}+\delta_{z y}+\delta_{z x y}, \\
f_{z x y} & :=\delta_{1}-\delta_{x}+\delta_{y}-\delta_{x y}-\delta_{z}+\delta_{z x}-\delta_{z y}+\delta_{z x y} .
\end{aligned}
$$

Then the map $H_{8} \rightarrow H_{8}^{*}$ given by $a \mapsto f_{a}$ for all $a \in \mathcal{B}$ and linear extension is a Hopf isomorphism.

Consequently, the Haar integrals $\phi_{B}$ read:

$$
\begin{aligned}
\phi_{\left\{f_{1}\right\}} & =\delta_{1}+\delta_{x}+\delta_{y}+\delta_{x y}+\delta_{z}+\delta_{z x}+\delta_{z y}+\delta_{z x y}=\epsilon, \\
\phi_{\left\langle f_{x}\right\rangle} & =\delta_{1}+\delta_{x}+\delta_{y}+\delta_{x y}, \\
\phi_{\left\langle f_{y}\right\rangle} & =\delta_{1}+\delta_{x y}+\frac{1+i}{2}\left(\delta_{z}+\delta_{z x y}\right)+\frac{1-i}{2}\left(\delta_{z x}+\delta_{z y}\right), \\
\phi_{\left\langle f_{x y}\right\rangle} & =\delta_{1}+\delta_{x y}+\frac{1-i}{2}\left(\delta_{z}+\delta_{z x y}\right)+\frac{1+i}{2}\left(\delta_{z x}+\delta_{z y}\right), \\
\phi_{\left\langle f_{x}, f_{y}\right\rangle} & =\delta_{1}+\delta_{x y}, \\
\phi & =\delta_{1} .
\end{aligned}
$$

\section{Constructing quantum double models from Hopf algebras}

The goal of this section is to construct a two-dimensional quantum spin model whose microscopic degrees of freedom are given by a finite-dimensional Hopf 
algebra $H$, such that its emerging degrees of freedom are characterized by the quantum double $\mathrm{D}(H)$. This illuminates Kitaev's insight in [26]. The model obtained in this fashion dynamically implements the quantum double construction. This means that its Hilbert space aquires additional structure, namely that the graph underlying the spin model can be interpreted as a $\mathrm{D}(H)$-module, or representation of $\mathrm{D}(H)$. State vectors describing (elementary) quasiparticle excitations above the ground state then live in the irreducible representations of $\mathrm{D}(H)$ the Hilbert space decomposes into. In fact, for every irreducible representation of the quantum double there will be a corresponding type of quasiparticle excitation. These quasiparticle excitations can then naturally be braided via the quasitriangular structure of $\mathrm{D}(H)$ and, in general, exhibit exchange statistics beyond bosons or fermions. In order to render their topological nature manifest we would also like these quasiparticle excitations to be agnostic to the details of the microscopic background. In particular, they should only feel the topological properties of the underlying surface rather than the precise shape of the embedded graph. In other words, the quantum spin model we construct should be insensitive to the particular discretization chosen. It goes without saying that any discretization will typically have a fine granularity in order to make the condensed matter character of the model evident.

Since there is a natural action of the quantum double $\mathrm{D}(H)$ on the Hopf algebra $H$ itself one can nevertheless regard a minimal graph consisting of precisely one edge as a representation of $\mathrm{D}(H)$ by identifying the edge with $H$. However, it is clear that this system cannot contain all irreducible representations of the quantum double. While this restriction can already be overcome by considering two graph edges associated with $H \otimes H$ one still needs to go to a macroscopic regime from either of these small graphs. It is precisely the process of spatially extending the action of $\mathrm{D}(H)$ from one edge to many edges which will eventually yield the Hamiltonian and thus define the quantum double model completely.

In the following we will first construct a representation of $\mathrm{D}(H)$ from a small graph with just two edges. Next we will endow any larger graph obtained from a surface cellulation with a local $\mathrm{D}(H)$-module structure. Finally we will introduce the Hilbert space for our generalized quantum double model and assemble the Hamiltonian from particular operators in the local representations of $\mathrm{D}(H)$.

3.1. Graph representations of quantum doubles. Given a finite-dimensional Hopf algebra $H$ and an oriented graph $\Gamma=(V, E, F)$ we define the microscopic Hilbert space as $\mathcal{N}_{\Gamma}=\bigotimes_{e \in E} H$. Our goal is to find local operators $A_{a}$ and $B_{f}$ acting on $\mathcal{N}_{\Gamma}$ which represent both parts $H$ and $X=\left(H^{\text {op }}\right)^{*}$ of the quantum double $\mathrm{D}(H)$ separately via the embeddings $i_{X}$ and $i_{H}$. Additionally we want these two operators to commute in such a way that they implement the straightening formula (43) of $\mathrm{D}(H)$. Put differently, we require the operators $A_{a}$ and $B_{f}$ to establish the bicrossed multiplication of the quantum double by interacting nontrivially on the intersection of their supports. Loosely speaking, this amounts to assembling the adjoint representation from smaller building blocks. Note that on the minimal graph comprising a single edge the adjoint representation can be turned into an operator "as is".

We begin with the small graph shown in Figure 1 and its associated Hilbert space $\mathcal{N}_{\Gamma}=H \otimes H$. In the spirit of [26] we define the following module structures 


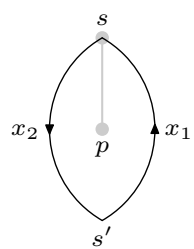

Fig. 1. Small graph representing $H \otimes H$. It affords a $\mathrm{D}(H)$-module structure at the site $(s, p)$ via the operators $A_{a}(s, p)$ and $B_{f}(s, p)$.

on $H$ for all $a, x \in H$ and $f \in X$ :

$$
\begin{aligned}
L_{+}^{a}(x) & :=a x, \\
L_{-}^{a}(x) & :=x S(a), \\
T_{+}^{f}(x) & :=\sum_{(x)}\left\langle f, x^{\prime \prime}\right\rangle x^{\prime}, \\
T_{-}^{f}(x) & :=\sum_{(x)}\left\langle f, S^{-1}\left(x^{\prime}\right)\right\rangle x^{\prime \prime} .
\end{aligned}
$$

More precisely, the operators $L_{ \pm}$define actions of $H$ on itself while $T_{ \pm}$define actions of $X$ on $H$. Furthermore these actions are intimately related: one may start with the left multiplication $L_{+}$and then canonically derive all other actions. As a consequence one has for example

$$
\begin{aligned}
& L_{-}^{a}=S \circ L_{+}^{a} \circ S^{-1}, \\
& T_{-}^{f}=S \circ T_{+}^{f} \circ S^{-1} .
\end{aligned}
$$

This means that if one fixes an arbitrary pattern of edge orientations one may relate any other pattern to the original one using these relations. Unfortunately this does not treat all possible orientations on equal footing. We will resolve this issue in Section 3.2. Also note that

$$
\begin{aligned}
{\left[L_{+}^{a}, L_{-}^{b}\right] } & =0, \\
{\left[T_{+}^{f}, T_{-}^{g}\right] } & =0
\end{aligned}
$$

for arbitrary $a, b \in H$ and $f, g \in X$.

Next we attribute operators $A_{a}$ and $B_{f}$ to the sites $(s, p)$ of the graph $\Gamma$. Following [26], a site is defined as a pair consisting of a vertex $s$ and one of its adjacent faces $p$. For the particular site $(s, p)$ shown in Figure 1 we define the operators in terms of the above actions:

$$
\begin{aligned}
& A_{a}(s, p):=\sum_{(a)} L_{+}^{a^{\prime}} \otimes L_{-}^{a^{\prime \prime}}, \\
& B_{f}(s, p):=\sum_{(f)} T_{-}^{f^{\prime}} \otimes T_{-}^{f^{\prime \prime}} .
\end{aligned}
$$

It is worth emphasizing that this definition only holds for the edge orientation chosen in the figure. However, using the conventions set forth by Figure 2 we can 


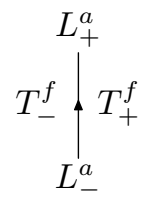

Fig. 2. A graph edge representing the Hopf algebra $H$ via the $H$-module structures $L_{ \pm}$. At the same time it represents the Hopf algebra $X=\left(H^{\mathrm{op}}\right)^{*}$ via the $X$-module structures $T_{ \pm}$. These are related to each other by means of the antipode in 106 and 107 .

extend this definition to arbitrarily oriented edges. Also note an important detail: our seemingly similar notation refers to two rather different comultiplications, namely, the one in $H$ and the other one in $X$.

We are now prepared to analyze the commutation properties of these operators which we take both at the same site $(s, p)$ for the moment. It is clear that the support of both operators coincides on this small graph, hence we can study the interaction of $A_{a}$ and $B_{f}$ without any additional complications. The following lemma shows that indeed the two operators define a representation of $\mathrm{D}(H)$ on $H \otimes H$.

Lemma 1. For the operators $A_{a}(s, p)$ and $B_{f}(s, p)$ the straightening formula (43) holds in the form:

$$
A_{a} B_{f}=\sum_{(a)} B_{f\left(S^{-1}\left(a^{\prime \prime \prime}\right) ? a^{\prime}\right)} A_{a^{\prime \prime}}
$$

Proof. The statement is proven by evaluating both sides of the straightening formula on arbitrary elements $x_{1}, x_{2} \in H$. To this end consider first:

$$
\begin{aligned}
A_{a} B_{f}\left(x_{1} \otimes x_{2}\right) & =A_{a} \sum_{(f)} T_{-}^{f^{\prime}}\left(x_{1}\right) \otimes T_{-}^{f^{\prime \prime}}\left(x_{2}\right) \\
& =A_{a} \sum_{\left(x_{i}\right)} \sum_{(f)}\left\langle f^{\prime}, S^{-1}\left(x_{1}^{\prime}\right)\right\rangle\left\langle f^{\prime \prime}, S^{-1}\left(x_{2}^{\prime}\right)\right\rangle x_{1}^{\prime \prime} \otimes x_{2}^{\prime \prime} \\
& =\sum_{\left(x_{i}\right)}\left\langle\sum_{(f)} f^{\prime} \otimes f^{\prime \prime}, S^{-1}\left(x_{1}^{\prime}\right) \otimes S^{-1}\left(x_{2}^{\prime}\right)\right\rangle \sum_{(a)} L_{+}^{a^{\prime}}\left(x_{1}^{\prime \prime}\right) \otimes L_{-}^{a^{\prime \prime}}\left(x_{2}^{\prime \prime}\right) \\
& =\sum_{(a)\left(x_{i}\right)}\left\langle f, S^{-1}\left(x_{2}^{\prime}\right) S^{-1}\left(x_{1}^{\prime}\right)\right\rangle a^{\prime} x_{1}^{\prime \prime} \otimes x_{2}^{\prime \prime} S\left(a^{\prime \prime}\right) \\
& =\sum_{(a)\left(x_{i}\right)}\left\langle f, S^{-1}\left(x_{1}^{\prime} x_{2}^{\prime}\right)\right\rangle a^{\prime} x_{1}^{\prime \prime} \otimes x_{2}^{\prime \prime} S\left(a^{\prime \prime}\right) .
\end{aligned}
$$

Note that in the third line we have used the opposite multiplication $\mu^{\text {op }}$ which is the appropriate dual to swap the comultiplication

$$
\sum_{(f)} f^{\prime} \otimes f^{\prime \prime}=\left(\mu^{\mathrm{op}}\right)^{T}(f)
$$

in $X$ from $f$ onto its argument in $H$. 
In a second step compare this with:

$$
\begin{aligned}
\sum_{(a)} B_{f\left(S^{-1}\left(a^{\prime \prime \prime}\right) ? a^{\prime}\right)} A_{a^{\prime \prime}}\left(x_{1} \otimes x_{2}\right) & =\sum_{(a)} B_{f\left(S^{-1}\left(a^{(4)}\right) ? a^{(1)}\right)}\left(a^{(2)} x_{1} \otimes x_{2} S\left(a^{(3)}\right)\right) \\
& =\sum_{(a)} B_{\tilde{f}}\left(a^{(2)} x_{1} \otimes x_{2} S\left(a^{(3)}\right)\right)
\end{aligned}
$$

where we used the temporary abbreviation $\tilde{f}=f\left(S^{-1}\left(a^{(4)}\right) ? a^{(1)}\right)$. Now we have for the right-hand side

$$
\begin{aligned}
& \sum_{(a)(\tilde{f})} T_{-}^{\tilde{f}^{\prime}}\left(a^{(2)} x_{1}\right) \otimes T_{-}^{\tilde{f}^{\prime \prime}}\left(x_{2} S\left(a^{(3)}\right)\right) \\
= & \sum_{(a)(\tilde{f})} \sum_{\left(a^{(2)} x_{1}\right)}\left\langle\tilde{f}^{\prime}, S^{-1}\left(\left(a^{(2)} x_{1}\right)^{\prime}\right)\right\rangle\left(a^{(2)} x_{1}\right)^{\prime \prime} \otimes \\
& \sum_{\left(x_{2} S\left(a^{(3)}\right)\right)}\left\langle\tilde{f}^{\prime \prime}, S^{-1}\left[\left(x_{2} S\left(a^{(3)}\right)\right)^{\prime}\right]\right\rangle\left(x_{2} S\left(a^{(3)}\right)\right)^{\prime \prime} \\
= & \sum_{\left(a^{(2)} x_{1}\right)} \sum_{\left(x_{2} S\left(a^{(3)}\right)\right)}\left\langle\tilde{f}, S^{-1}\left[\left(x_{2} S\left(a^{(3)}\right)\right)^{\prime}\right] S^{-1}\left(\left(a^{(2)} x_{1}\right)^{\prime}\right)\right\rangle \\
= & \sum_{(a)} \sum_{\left(a^{(2)} x_{1}\right)} \sum_{\left(x_{2} S\left(a^{(3)}\right)\right)}\left\langle\tilde{f}, S^{-1}\left[\left(a^{(2)} x_{1}\right)^{\prime}\left(x_{2} S\left(a^{(3)}\right)\right)^{\prime}\right]\right\rangle\left(a^{(2)} x_{1}\right)^{\prime \prime} \otimes\left(x_{2} S\left(a^{(3)}\right)\right)^{\prime \prime}
\end{aligned}
$$

and upon restoring $f$ this becomes

$$
\begin{aligned}
& \sum_{(a)} \sum_{\left(a^{(2)} x_{1}\right)} \sum_{\left(x_{2} S\left(a^{(3)}\right)\right)}\left\langle f, S^{-1}\left(a^{(4)}\right) S^{-1}\left[\left(a^{(2)} x_{1}\right)^{\prime}\left(x_{2} S\left(a^{(3)}\right)\right)^{\prime}\right] a^{(1)}\right\rangle \\
= & \sum_{(a)} \sum_{\left(a^{(2)} x_{1}\right)} \sum_{\left(x_{2} S\left(a^{(3)}\right)\right)}\left\langle f, S^{-1}\left[\left(a^{(2)} x_{1}\right)^{\prime}\left(x_{2} S\left(a^{(3)}\right)\right)^{\prime} a^{(4)}\right] a^{(1)}\right\rangle \\
= & \left.\sum_{(a)\left(x_{i}\right)} \sum_{\left(a^{(2)}\right)} \sum_{\left(S\left(a^{(3)}\right)\right)}\left\langle f, S^{-1}\left(\left(a^{(2)} x_{1}\right)^{\prime \prime} \otimes\left(a_{2} S\left(a^{(2)}\right)^{\prime} x_{1}^{\prime} x_{2}^{\prime} S\left(a^{(3)}\right)\right)^{\prime \prime}\right)^{\prime} a^{(4)}\right) a^{(1)}\right\rangle \\
= & \sum_{(a)\left(x_{i}\right)} \sum_{\left(S\left(a^{(4)}\right)\right)}\left\langle f, S^{-1}\left(a^{(2)}\right)^{\prime \prime} x_{1}^{\prime \prime} \otimes x_{2}^{\prime \prime} x_{2}^{\prime} S\left(a^{(3)}\right)^{\prime \prime}\right. \\
= & \sum_{(a)\left(x_{i}\right)} \sum_{\left(a^{(4)}\right)}\left\langle f, S^{-1}\left[a^{(5)}\right) a^{(1)} x_{1}^{\prime} x_{2}^{\prime} S\left(\left(a^{(4)}\right)^{\prime \prime}\right) a^{(3)} x_{1}^{\prime \prime} \otimes x_{2}^{\prime \prime} S\left(a^{(4)}\right)^{\prime \prime}\right. \\
= & \sum_{(a)\left(x_{i}\right)} f\left[S ^ { - 1 } \left(a ^ { ( 2 ) } x _ { 1 } ^ { \prime } x _ { 2 } ^ { \prime } S \left(a^{(3)} x_{1}^{\prime \prime} \otimes x_{2}^{\prime \prime} S\left(\left(a^{(4)}\right)^{\prime}\right)\right.\right.\right. \\
= & \left.\sum_{(a)\left(x_{i}\right)} f\left[S^{-1}\left(a^{(2)} x_{1}^{\prime} a^{(1)}\right] a^{(3)} x_{1}^{\prime \prime} \otimes x_{2}^{\prime \prime} S\left(a^{(5)}\right)\right) a^{(1)}\right] a^{(3)} x_{1}^{\prime \prime} \otimes x_{2}^{\prime \prime} S\left(a^{(4)}\right)
\end{aligned}
$$




$$
\begin{aligned}
& =\sum_{(a)\left(x_{i}\right)} f\left(S^{-1}\left(x_{1}^{\prime} x_{2}^{\prime}\right) S^{-1}\left(a^{(2)}\right) a^{(1)}\right) a^{(3)} x_{1}^{\prime \prime} \otimes x_{2}^{\prime \prime} S\left(a^{(4)}\right) \\
& =\sum_{(a)\left(x_{i}\right)} f\left(S^{-1}\left(x_{1}^{\prime} x_{2}^{\prime}\right) \epsilon\left(a^{\prime}\right)\right) a^{\prime \prime} x_{1}^{\prime \prime} \otimes x_{2}^{\prime \prime} S\left(a^{\prime \prime \prime}\right) \\
& =\sum_{(a)\left(x_{i}\right)}\left\langle f, S^{-1}\left(x_{1}^{\prime} x_{2}^{\prime}\right)\right\rangle a^{\prime} x_{1}^{\prime \prime} \otimes x_{2}^{\prime \prime} S\left(a^{\prime \prime}\right) .
\end{aligned}
$$

Note that in the third line we have employed the fact that $\Delta$ is an algebra morphism. Since the above is true for all $x_{1}, x_{2} \in H$ we have just proven the claim.

In order to obtain local $\mathrm{D}(H)$-representations at the sites of arbitrary graphs we need to extend the above actions of $A_{a}$ and $B_{f}$ to larger vertices and faces. It will inevitably happen that the two operators $A_{a}$ and $B_{f}$ act on different sets of edges which do not fully coincide. We need to ensure that a) both operators continue to represent their respective parts $H$ and $X$ individually and b) the commutation relation arising from common edges still implements the bicrossed structure of the quantum double $\mathrm{D}(H)$.

The extension can be done as follows for general vertices $s$ and faces $p$.

Definition 1. Let $(s, p)$ be a site of the graph $\Gamma, a \in H$ and $f \in X$. Then we define vertex and face operators via

$$
\begin{aligned}
& A_{a}(s, p) \cdots \longrightarrow x_{x_{r}}:=\sum_{(a)} L_{+}^{a^{(1)}}\left(x_{1}\right) \otimes \cdots \otimes L_{+}^{a^{(r)}}\left(x_{r}\right) \\
& =\sum_{(a)} \ldots \longrightarrow a_{a^{(r)} x_{r}}^{a^{(2)} x_{2}} \\
& B_{f}(s, p) x_{r} \underbrace{\dddot{p}}_{x_{1}} \underbrace{x_{2}}:=\sum_{(f)} T_{-}^{f^{(r)}}\left(x_{1}\right) \otimes \cdots \otimes T_{-}^{f^{(1)}}\left(x_{r}\right)
\end{aligned}
$$

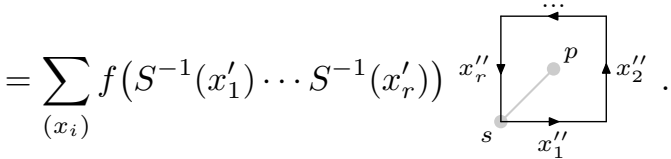

Note that for $A_{a}(s, p)$ the vertex $s$ denotes the centre of the loop on the dual graph and $p$ denotes the starting point within the loop for comultiplication. Analogously, for $B_{f}(s, p)$ the starting point of the loop around the face $p$ is 


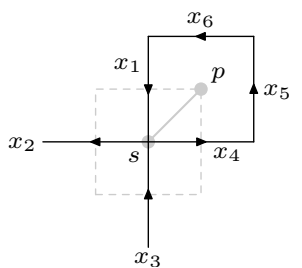

Fig. 3. Graph used in the proof of Theorem 1 The commutation relation and hence the structure of $\mathrm{D}(H)$ is determined on the intersection of the supports of $A_{a}(s, p)$ and $B_{f}(s, p)$.

marked by $s$. For different edge orientations the actions $L_{+}$and $T_{-}$in 1113 and (114) may need to be replaced per edge by $L_{-}$and $T_{+}$according to Figure 2.

The idea behind this is: first one fixes the action of $a \in H$ such that the coproduct of $a$ is applied to the edges of the vertex $s$ in counterclockwise order. This is indicated by the arrow winding around $s$. When defining the action of $f \in X$ on a face it then appears at first there might be two choices for the orientation of the coproduct of $f$. It turns out that only one of them, namely distributing the coproduct of $f$ in clockwise orientation on the graph, will yield a meaningful theory. The orientation of the coproduct is again indicated by the arrow winding around $p$.

At this point the reader may wonder why one does not take the function $f$ directly from $H^{*}$ (the dual of $H$ without flipped comultiplication) and the following more symmetric definition of the action (114):

$$
\begin{aligned}
\widetilde{B}_{f}(s, p) x_{r} \overbrace{s}^{p} x_{2} & :=\sum_{(f)} T_{-}^{f^{(1)}}\left(x_{1}\right) \otimes \cdots \otimes T_{-}^{f^{(r)}}\left(x_{r}\right) \\
& =\sum_{\left(x_{i}\right)} f\left(S^{-1}\left(x_{1}^{\prime}\right) \cdots S^{-1}\left(x_{r}^{\prime}\right)\right)
\end{aligned}
$$

Note that here the arrow winds around $p$ in counterclockwise orientation since $f \in H^{*}$ has a different coproduct now as compared to (114). Clearly, both (114) and (115) define the same (algebra) action on the boundary edges of the face. However, as cumbersome as taking $f \in X$ in the definition of $B_{f}(s, p)$ may seem it will make the next task tremendously easier: making contact with the Drinfeld double $\mathrm{D}(H)=X \bowtie H$ which these operators $A_{a}$ and $B_{f}$ represent.

Theorem 1. Let $H$ a finite-dimensional Hopf algebra. Then each site $(s, p)$ of the graph $\Gamma$ supports a $\mathrm{D}(H)$-module structure given by the operators $A_{a}(s, p)$ and $B_{f}(s, p)$ from Definition 1 .

Proof. In order to keep the notation simple we only consider sites such as the one depicted in Figure 3. The generality of the argument will not be affected.

In order to prove the $\mathrm{D}(H)$-module structure at site $(s, p)$ it is again enough to show that the straightening formula holds. Let $a \in H, f \in X$ and evaluate 
$A_{a} B_{f}$ on arbitrary edges $x_{i} \in H$ :

$$
\begin{aligned}
A_{a} B_{f}\left(x_{1} \otimes \cdots \otimes x_{6}\right)= & A_{a} \sum_{\left(x_{i}\right)} f\left(S^{-1}\left(x_{4}^{\prime}\right) S^{-1}\left(x_{5}^{\prime}\right) S^{-1}\left(x_{6}^{\prime}\right) S^{-1}\left(x_{1}^{\prime}\right)\right) \\
& x_{1}^{\prime \prime} \otimes x_{2} \otimes x_{3} \otimes x_{4}^{\prime \prime} \otimes x_{5}^{\prime \prime} \otimes x_{6}^{\prime \prime} \\
= & \sum_{\left(x_{i}\right)} f\left(S^{-1}\left(x_{4}^{\prime}\right) S^{-1}\left(x_{5}^{\prime}\right) S^{-1}\left(x_{6}^{\prime}\right) S^{-1}\left(x_{1}^{\prime}\right)\right) \\
& \sum_{(a)} a^{(1)} x_{1}^{\prime \prime} \otimes x_{2} S\left(a^{(2)}\right) \otimes a^{(3)} x_{3} \otimes x_{4}^{\prime \prime} S\left(a^{(4)}\right) \otimes x_{5}^{\prime \prime} \otimes x_{6}^{\prime \prime} \\
= & \sum_{(a)\left(x_{i}\right)}\left\langle f, S^{-1}\left(x_{1}^{\prime} x_{6}^{\prime} x_{5}^{\prime} x_{4}^{\prime}\right)\right\rangle \\
& a^{(1)} x_{1}^{\prime \prime} \otimes x_{2} S\left(a^{(2)}\right) \otimes a^{(3)} x_{3} \otimes x_{4}^{\prime \prime} S\left(a^{(4)}\right) \otimes x_{5}^{\prime \prime} \otimes x_{6}^{\prime \prime} .
\end{aligned}
$$

Compare this with the case:

$$
\begin{aligned}
& \sum_{(a)} B_{f\left(S^{-1}\left(a^{\prime \prime \prime}\right) ? a^{\prime}\right)} A_{a^{\prime \prime}}\left(x_{1} \otimes \cdots \otimes x_{6}\right) \\
& =\sum_{(a)} B_{f\left(S^{-1}\left(a^{(6)}\right) ? a^{(1)}\right)} a^{(2)} x_{1} \otimes x_{2} S\left(a^{(3)}\right) \otimes a^{(4)} x_{3} \otimes x_{4} S\left(a^{(5)}\right) \otimes x_{5} \otimes x_{6} .
\end{aligned}
$$

Again, abbreviating $\tilde{f}=f\left(S^{-1}\left(a^{(6)}\right) ? a^{(1)}\right)$ we obtain for the right-hand side

$$
\begin{aligned}
& \sum_{(a)} \sum_{\left(x_{4} S\left(a^{(5)}\right)\right)} \sum_{\left(x_{5}\right)\left(x_{6}\right)} \sum_{\left(a^{(2)} x_{1}\right)}\left\langle\tilde{f}, S^{-1}\left[\left(x_{4} S\left(a^{(5)}\right)\right)^{\prime}\right] S^{-1}\left(x_{5}^{\prime}\right) S^{-1}\left(x_{6}^{\prime}\right) S^{-1}\left(\left(a^{(2)} x_{1}\right)^{\prime}\right)\right\rangle \\
& \left(a^{(2)} x_{1}\right)^{\prime \prime} \otimes x_{2} S\left(a^{(3)}\right) \otimes a^{(4)} x_{3} \otimes\left(x_{4} S\left(a^{(5)}\right)\right)^{\prime \prime} \otimes x_{5}^{\prime \prime} \otimes x_{6}^{\prime \prime} \\
& =\sum_{(a)} \sum_{\left(x_{i}\right)} \sum_{\left(a^{(2)}\right)} \sum_{\left(a^{(5)}\right)}\left\langle\tilde{f}, S^{-1}\left[x_{4}^{\prime} S\left(\left(a^{(5)}\right)^{\prime \prime}\right)\right] S^{-1}\left(x_{5}^{\prime}\right) S^{-1}\left(x_{6}^{\prime}\right) S^{-1}\left(\left(a^{(2)}\right)^{\prime} x_{1}^{\prime}\right)\right\rangle \\
& =\sum_{(a)\left(a_{i}\right)} \sum_{\left(a^{(2)}\right)} \sum_{\left(a^{(5)}\right)}\left\langle\tilde{f}, S^{-1}\left[\left(a^{(2)}\right)^{\prime} x_{1}^{\prime} x_{6}^{\prime} x_{5}^{\prime} x_{4}^{\prime} S\left(\left(a^{(5)}\right)^{\prime \prime}\right)\right]\right\rangle \\
& \left(a^{(2)}\right)^{\prime \prime} x_{1}^{\prime \prime} \otimes x_{2} S\left(a^{(3)}\right) \otimes a^{(4)} x_{3} \otimes x_{4}^{\prime \prime} S\left(\left(a^{(5)}\right)^{\prime}\right) \otimes x_{5}^{\prime \prime} \otimes x_{6}^{\prime \prime}
\end{aligned}
$$

and now restoring $f$ this becomes

$$
\begin{aligned}
& \sum_{(a)\left(x_{i}\right)}\left\langle f\left(S^{-1}\left(a^{(8)}\right) ? a^{(1)}\right), S^{-1}\left(a^{(2)} x_{1}^{\prime} x_{6}^{\prime} x_{5}^{\prime} x_{4}^{\prime} S\left(a^{(7)}\right)\right)\right\rangle \\
& a^{(3)} x_{1}^{\prime \prime} \otimes x_{2} S\left(a^{(4)}\right) \otimes a^{(5)} x_{3} \otimes x_{4}^{\prime \prime} S\left(a^{(6)}\right) \otimes x_{5}^{\prime \prime} \otimes x_{6}^{\prime \prime} \\
& =\sum_{(a)\left(x_{i}\right)}\left\langle f, S^{-1}\left(a^{(8)}\right) a^{(7)} S^{-1}\left(x_{1}^{\prime} x_{6}^{\prime} x_{5}^{\prime} x_{4}^{\prime}\right) S^{-1}\left(a^{(2)}\right) a^{(1)}\right\rangle \\
& a^{(3)} x_{1}^{\prime \prime} \otimes x_{2} S\left(a^{(4)}\right) \otimes a^{(5)} x_{3} \otimes x_{4}^{\prime \prime} S\left(a^{(6)}\right) \otimes x_{5}^{\prime \prime} \otimes x_{6}^{\prime \prime}
\end{aligned}
$$




$$
\begin{aligned}
= & \sum_{(a)\left(x_{i}\right)}\left\langle f, S^{-1}\left(x_{1}^{\prime} x_{6}^{\prime} x_{5}^{\prime} x_{4}^{\prime}\right) S^{-1}\left(a^{(2)}\right) a^{(1)}\right\rangle \\
& a^{(3)} x_{1}^{\prime \prime} \otimes x_{2} S\left(a^{(4)}\right) \otimes a^{(5)} x_{3} \otimes x_{4}^{\prime \prime} S\left(a^{(6)}\right) \otimes x_{5}^{\prime \prime} \otimes x_{6}^{\prime \prime} \\
= & \sum_{(a)\left(x_{i}\right)}\left\langle f, S^{-1}\left(x_{1}^{\prime} x_{6}^{\prime} x_{5}^{\prime} x_{4}^{\prime}\right)\right\rangle \\
& a^{(1)} x_{1}^{\prime \prime} \otimes x_{2} S\left(a^{(2)}\right) \otimes a^{(3)} x_{3} \otimes x_{4}^{\prime \prime} S\left(a^{(4)}\right) \otimes x_{5}^{\prime \prime} \otimes x_{6}^{\prime \prime} .
\end{aligned}
$$

This proves the statement.

3.2. Hilbert space. In order to obtain a physical system from the above discussion we need to define both a Hilbert space and a Hamiltonian for our topological lattice model. In particular, we need to find Hopf $*$-algebras $H$ that allow for an inner product and $*$-representations. This means that adjoint operators (which as usual are defined relative to the inner product) in a representation of $H$ are compatible with the $*$-structure of $H$ itself. It turns out we can reach both goals by requiring $H$ to be a finite-dimensional Hopf $C^{*}$-algebra. Such a Hopf algebra comes endowed with a unique element called the Haar integral which will naturally define both the inner product and the Hamiltonian.

In order to define the Hilbert space we begin with the following proposition which is obtained from [37,29].

Proposition 1. Let $H$ be a finite-dimensional Hopf $C^{*}$-algebra. Then $S^{2}=\mathrm{id}$ and there exists a unique two-sided integral $h \in H$ with the following properties:

1. $h^{2}=h$,

2. $h^{*}=h$,

3. $S(h)=h$,

4. $h \in \operatorname{Cocom}(H)$.

Furthermore, $H^{*}$ is a Hopf $C^{*}$-algebra again and its unique integral $\phi \in H^{*}$ satisfying 1-4 is a faithful positive functional, or trace, on $\mathrm{H}$.

As a first consequence we can easily resolve the issue of edge orientation: since the antipode is now involutive we define the reversal of an edge $e$ simply by

$$
x_{e} \mapsto S\left(x_{e}\right) .
$$

This is obviously compatible with the actions (102), 103, (104) and 105). At the same time we no longer need to pick a distinguished pattern of edge orientations, rather all patterns are equivalent.

Although the preceding proposition tells us that $\phi$ is a positive trace on $H$, we need to know its precise relationship with the usual $\operatorname{trace} \operatorname{tr}_{H}(a)=\operatorname{tr}\left(L_{+}^{a}\right)$ found in the literature on Hopf algebras. Setting $|H|:=\operatorname{dim} H$ we have

Lemma 2. Let $H$ a finite-dimensional Hopf $C^{*}$-algebra. Then

$$
\operatorname{tr}_{H}=|H| \cdot \phi
$$

holds where $\phi \in H^{*}$ is the Haar functional on $H$. 
Proof. By the above Proposition we know that $S^{2}=$ id. In this case we have

$$
\operatorname{tr}_{H}=\epsilon(\Lambda) \phi
$$

for the integral $\Lambda \in H$ which is normalized such that $\phi(\Lambda)=140$. Since $\phi(h)=|H|^{-1}$ for the Haar integral $h \in H[29$ we actually have that $\Lambda=|H| h$. Finally, $\epsilon(h)=1$ concludes the proof.

Since $\phi$ is a faithful positive trace on $H$ we can derive a Hermitian inner product on $H$ from it by setting

$$
(a, b)_{H}=\phi\left(a^{*} b\right) .
$$

This inner product now turns both the module structures $L_{ \pm}$and $T_{ \pm}$on $H$ into $*$-representations. Indeed, relative to 119 the adjoint map of $L_{ \pm}^{a}$ is given by $\left(L_{ \pm}^{a}\right)^{\dagger}=L_{ \pm}^{a^{*}}$ because of

$$
\left(x, L_{+}^{a}(y)\right)=\phi\left(x^{*} a y\right)=\left\langle\phi,\left(a^{*} x\right)^{*} b\right\rangle=\left(L_{+}^{a^{*}}(x), y\right) .
$$

An easy but tedious calculation shows that $\left(T_{ \pm}^{f}\right)^{\dagger}=T_{ \pm}^{f^{*}}$ holds, too. Remember that $f^{*}$ is given by (51).

This means that for the operators $A_{a}$ and $B_{f}$ which represent the Drinfeld double $\mathrm{D}(H)$ the adjoint operators are given by:

$$
\begin{aligned}
& A_{a}^{\dagger}(s, p)=A_{a^{*}}(s, p), \\
& B_{f}^{\dagger}(s, p)=B_{f^{*}}(s, p) .
\end{aligned}
$$

3.3. Hamiltonian. It remains to specify a Hamiltonian for the model. In analogy to 26 we would like to get a frustration-free Hamiltonian, i.e. a sum of commuting terms, and we would like to derive it from the local operators $A_{a}$ and $B_{f}$ defined previously. Hence we need to identify a subset of these operators such that they mutually commute with each other.

Before anything else it is natural to analyze the commutation relation between $A_{a}$ and $B_{f}$ at the same site $(s, p)$ of the graph $\Gamma$. Suppose in the following that both $a \in H$ and $f \in X$ are such that $\langle f g, a\rangle=\langle g f, a\rangle$ for any $f, g \in X$ and $f(x y)=f(y x)$ for any $x, y \in H{ }^{6}$ Then at the level of $\mathrm{D}(H)$ we have

$$
\begin{aligned}
a f & =\sum_{(a)} f\left(S^{-1}\left(a^{\prime \prime \prime}\right) ? a^{\prime}\right) a^{\prime \prime} \\
& =\sum_{(a)} f\left(a^{\prime} S^{-1}\left(a^{\prime \prime \prime}\right) ?\right) a^{\prime \prime} \\
& =\sum_{(a)} f\left(a^{\prime \prime \prime} S^{-1}\left(a^{\prime \prime}\right) ?\right) a^{\prime} \\
& =\sum_{(a)} f\left(\epsilon\left(a^{\prime \prime}\right) ?\right) a^{\prime} \\
& =f a,
\end{aligned}
$$

\footnotetext{
6 It is easy to see that this is equivalent to $a \in \operatorname{Cocom}(H)$ and $f \in \operatorname{Cocom}(X)$. Clearly, $\operatorname{Cocom}(H)$ and $\operatorname{Cocom}(X)$ are defined relative to the respective coproducts in $H$ and $X$, which are fundamentally different.
} 
where we used the cocommutativity of $f$ (or $a$ respectively) in the second (third) line and the skew-antipode $S^{-1}$ in the fourth one. In other words, the straightening formula 43 becomes trivial for such elements. Since for a fixed site $(s, p)$ the operators $A_{a}(s, p)$ and $B_{f}(s, p)$ form a representation of $\mathrm{D}(H)$ this commutation relation immediately carries over to:

$$
A_{a}(s, p) B_{f}(s, p)=B_{f}(s, p) A_{a}(s, p) .
$$

Interestingly, with this choice of $a$ and $f$ the full specification of a site $(s, p)$ becomes unnecessary for the operators $A_{a}$ and $B_{f}$. Indeed, since coproducts can now be permuted cyclically, the starting point $p$ of the dual loop used to define the vertex operator $A_{a}(s, p)$ loses any significance. All that matters for the action is the vertex $s$ itself. In order to reflect this fact we set for all $a \in \operatorname{Cocom}(H)$ :

$$
A_{a}(s):=A_{a}(s, p) .
$$

By the same token a face operator $B_{f}(s, p)$ only cares about the face $p$ once we choose $f \in \operatorname{Cocom}(X)$ :

$$
B_{f}(p):=B_{f}(s, p) .
$$

Having restricted the possible candidates for the terms in the Hamiltonian by exploiting the $\mathrm{D}(H)$-module structure at a single fixed site we need to ensure subsequently that all vertex operators $A_{a}(s)$ commute among themselves, too. Observe first that

$$
\left[A_{a}(s), A_{b}\left(s^{\prime}\right)\right]=0
$$

for any $a, b \in H$ whenever two vertices $s$ and $s^{\prime}$ do not coincide. Indeed, if $A_{a}(s)$ acts on a common edge via $L_{+}$then $A_{b}\left(s^{\prime}\right)$ acts on the same edge via $L_{-}$and vice versa, hence both operators commute by (108). If there are no common edges then there is nothing to show. On the other hand if $s=s^{\prime}$ then the edge set $E(s)$ has an $H$-module structure (inherited from the $\mathrm{D}(H)$-module structure at any site that contains $s$ ) which implies

$$
\left[A_{a}(s), A_{b}(s)\right]=A_{[a, b]}(s) .
$$

This suggests to further narrow down our set of candidate vertex operators $A_{a}(s)$ by additionally requiring $a \in \mathrm{Z}(H)$ where $\mathrm{Z}(H)$ denotes the centre of the Hopf algebra $H$. By analogy, we are naturally led to assume $f \in \mathrm{Z}(X) \cap \operatorname{Cocom}(X)$ in the following if we want all face operators $B_{f}(p)$ to commute.

Finally we would like to remark that $A_{a}(s)$ and $B_{f}(p)$ trivially commute if the pair $(s, p)$ is not a site. In fact, the sets of edges they act on are even disjoint in this case.

Recalling the properties of the Haar integral from Proposition 1 we now state the main result of this section.

Theorem 2 (Generalized quantum double model). Let $H$ a finite-dimensional Hopf $C^{*}$-algebra with Haar integral $h$ and Haar functional $\phi$ and let $\Gamma$ a graph. Furthermore for each $s \in V$ and $p \in F$ define the projectors

$$
\begin{aligned}
& A(s):=A_{h}(s, p), \\
& B(p):=B_{\phi}(s, p) .
\end{aligned}
$$


Then

$$
\mathcal{H}=-\sum_{s \in V} A(s)-\sum_{p \in F} B(p)
$$

is a local, frustration-free Hamiltonian defining the $\mathrm{D}(H)$-model.

Proof. First observe that

$$
A(s)^{2}=A_{h^{2}}(s, p)=A(s)
$$

by Proposition 1. The same argument shows that $B(p)$ is a projector, too.

By the preceding discussion and Proposition 1 it is clear that all local terms $A(s)$ and $B(p)$ commute with each other. Furthermore, $\sqrt{121}$ and $\sqrt{122}$ imply that they are Hermitian.

For the reader's convenience we give a short summary of how these operators act on the graph:

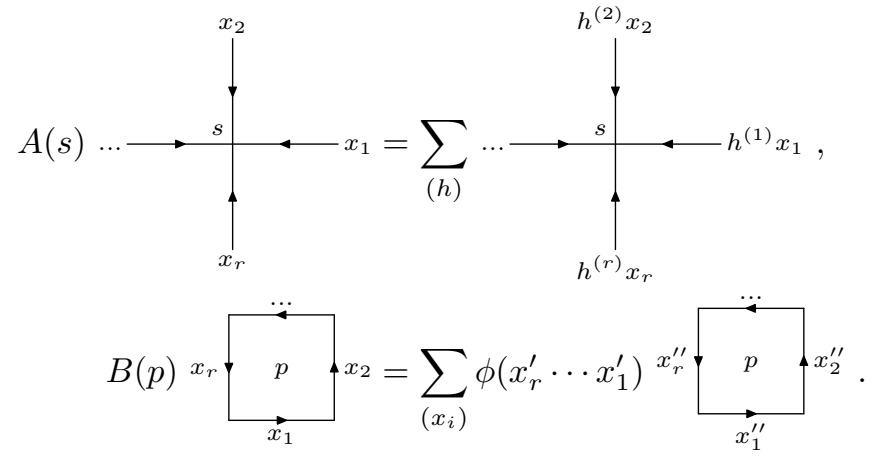

Alternatively, for a different orientation of the graph edges the face operator acts as follows:

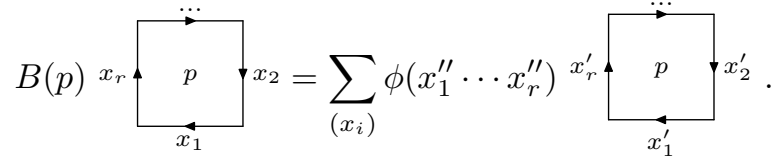

It is no coincidence that already at this stage do the Haar integrals of $H$ and $H^{*}$ reveal themselves as the crucial ingredients for the generalized quantum double models. Since we set out to construct a quantum spin model whose (elementary) quasiparticle excitations are characterized by irreducible representations of $\mathrm{D}(H)$ the ground state sector with no quasiparticles present necessarily has the structure of a trivial representation of $\mathrm{D}(H)$ locally. Precisely this is what the element $\phi \otimes h \in \mathrm{D}(H)$ embodies. As the Hamiltonian intrinsically encodes information about the ground state sector it should not surprise the reader that the Haar integrals $h$ and $\phi$ play such a prominent role. 
3.4. Comparison with $\mathrm{D}(\mathbb{C} G)$-models. We would now like to briefly comment on the relation between our generalized quantum double models and Kitaev's original construction in [26].

First note that in the case $H=\mathbb{C} G$ the terms given by 132 and 133 in the above Hamiltonian reduce to the operators of (1) and (2) which are the ones Kitaev employed for his quantum double models based on a group $G$. This becomes clear from Section 2.4.1 where the relevant expressions for $h$ and $\phi$ are listed.

Secondly, the entire theory of ribbon operators readily carries over from 26$]$ to our generalized quantum double models. This is because ribbon operators are constructed from certain elementary operators associated with two types of triangles any given ribbon path decomposes into. These operators are nothing but the $L_{ \pm}$and $T_{ \pm}$that implement the $H$ - and $X$-module structures. Furthermore patching together a ribbon operator from those elementary pieces only involves the structure maps of the Hopf algebra $H$ itself. Actually, we even used ribbon paths in order to define the local $\mathrm{D}(H)$-module structures (and hence the Hamiltonian) without saying so.

Finally, our generalized quantum double models inherit all the beautiful topological properties of the original since these follow exclusively from the algebraic structure of ribbon operators. In particular, these features include the degeneracy of the ground state sector as well as the exotic statistics of the quasiparticle excitations whose anyonic nature is revealed via braiding and fusion operations.

\section{A hierarchy of topological tensor network states}

In this section we develop a general diagrammatic language for tensor network states built from finite-dimensional Hopf $C^{*}$-algebras. Underlying surfaces both with and without boundaries are considered and we show how to naturally describe subsystems. Using this framework we solve the generalized quantum double model introduced in the preceding section by providing a tensor network representation for one of its ground states. Any other energy eigenstate can be obtained from there by an appropriate ribbon operator. The tensor network representation for that ground state only involves the canonical structures of the underlying Hopf $C^{*}$-algebra $H$ and its dual: multiplication, comultiplication, antipode and Haar integral. This insight leads us to propose a novel hierarchy of tensor network states based on Hopf subalgebras. For $H=\mathbb{C} G$ we are able to completely classify this hierarchy of states in terms of charge condensation. Finally we describe the relation between our Hopf tensor network language and the usual formulation of PEPS.

Unless otherwise noted, from now on $H$ will be a finite-dimensional Hopf $C^{*}$-algebra with Haar integral $h \in H$ and Haar functional $\phi \in H^{*}$.

4.1. Tensor traces. As we discussed in the introduction, the fully contracted tensor network (which is a complex number) for a certain ground state of the $\mathrm{D}(\mathbb{C} G)$-model on the graph $\Gamma$ can be interpreted as a collection of virtual loops in the faces of $\Gamma$ that are suitably glued together to form the physical degrees of freedom. 
We can extend this idea to the case of any finite-dimensional Hopf $C^{*}$ algebra $H$ now. In each face $p \in F$ place a virtual loop oriented in counterclockwise direction and associate a function $f_{p} \in X$ to this loop. For the moment we may restrict to $f_{p} \in \operatorname{Cocom}(X)$. With each oriented edge $e \in E$ we associate an algebra element $x_{e} \in H$ which splits into two parts as follows:

$$
((S \otimes \mathrm{id}) \circ \Delta)\left(x_{e}\right)=\sum_{\left(x_{e}\right)} S\left(x_{e}^{\prime}\right) \otimes x_{e}^{\prime \prime}
$$

Subsequently, we attribute $x_{e}^{\prime \prime}$ to the left adjacent face of $e$ and $S\left(x_{e}^{\prime}\right)$ to the right one. A virtual loop in face $p$ is then evaluated by taking the clockwise product of all elements thus associated with the loop from the surrounding edges of $p$. The result is then fed to the function $f_{p}$.

In order to simplify arguments we introduce a diagrammatic notation which encodes calculations with these tensor networks. In diagrammatic language the evaluation rule just described reads

$$
x_{e}:=\sum_{\left(x_{e}\right)} f_{p}\left(S\left(x_{e}^{\prime}\right) \ldots\right) f_{q}\left(x_{e}^{\prime \prime} \ldots\right) \text {. }
$$

Since $f_{p}$ is assumed cocommutative its argument can be permuted cyclically and we may start both virtual loops around $p$ and $q$ at the edge $e$ without loss of generality. The clockwise order of the product remains important though. Both the dashed lines and the ellipses denote the remaining degrees of freedom of the faces $p$ and $q$ respectively. Note that the glueing procedure generalizing (15) is implemented by the coproduct. We will introduce a full description of this later in 143 and (144). Finally, a reversed edge is resolved via

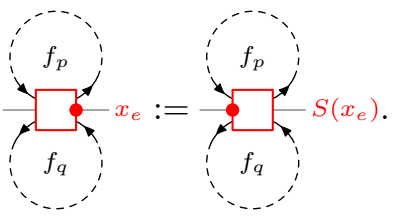

In summary we have the following

Definition 2 (Hopf tensor trace without boundary). For each $p \in F$ let $f_{p} \in \operatorname{Cocom}(X)$. Then the Hopf tensor trace associated with the graph $\Gamma$ is the function $\operatorname{ttr}_{\Gamma}: H^{\otimes|E|} \otimes X^{\otimes|F|} \rightarrow \mathbb{C}$,

$$
\bigotimes_{e \in E} x_{e} \bigotimes_{p \in F} f_{p} \mapsto \operatorname{ttr}_{\Gamma}\left(\left\{x_{e}\right\} ;\left\{f_{p}\right\}\right)
$$

which is defined via diagrams and the evaluation rules (136) and (137).

From this definition it is clear that the tensor trace is linear in each argument. It can be regarded as the wavefunction amplitude of a quantum many-body state as we will see shortly. 


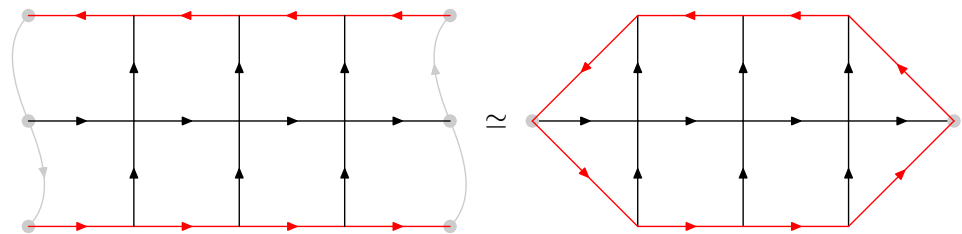

Fig. 4. Ordinary faces vs. boundary "faces". Wherever the boundary of the surface $M$ does not coincide with an edge of the graph $\Gamma$ it is displayed in grey. Upon smooth deformation of this boundary the grey vertices on either side can actually be identified with each other. This modifies $\Gamma$ and thus creates new faces whose preimages on the left-hand side we call boundary "faces". Boundary edges are drawn in red.

In the following we would like to investigate Hopf tensor networks on graphs which are embedded in a surface $M$ with boundary as well as Hopf tensor networks at interfaces between regions $R$ of a surface with or without boundary.

In general the edge set $E=E_{M}$ of the graph $\Gamma$ embedded in $M$ may be decomposed into the disjoint union of interior edges $E_{M} \circ$ and boundary edges $E_{\partial M}$ (see Figure 4). On the other hand, the set of faces $F=F_{M}=F_{M}$ o only contains interior faces by definition. However, one may collect incomplete faces (where two vertices of degree 1 can be connected/merged to yield a genuine new face) into the set $F_{\partial M}$ of boundary "faces" as illustrated in Figure 4 . By construction these are not faces of the original graph, hence $F \cap F_{\partial M}=\emptyset$.

Furthermore we may collect all boundary segments into the set $X_{\partial M}=E_{\partial M} \cup$ $F_{\partial M}$. Once we fix a distinguished segment on the boundary this set is equipped with a natural ordering inherited from the orientation of $\partial M$. Without loss of generality we will take any boundary to be oriented in counterclockwise direction with respect to its interior for the remainder of the article.

In order to take nontrivial boundaries into account we need to refine our diagrammatic notation for tensor networks. Pick an arbitrary edge $e \in E$. Since it belongs to the boundary of at least one face $p$ in either $F$ or $F_{\partial M}$ (i.e. $p$ is either an interior or a boundary face) we may define the following elementary diagram for any elements $f_{p} \in X$ and $x_{e} \in H$ simply as their canonical pairing:

$$
\overbrace{x_{e}}^{f_{p}}:=f_{p}\left(x_{e}\right) .
$$

Different orientations of graph edges and virtual loops are resolved via

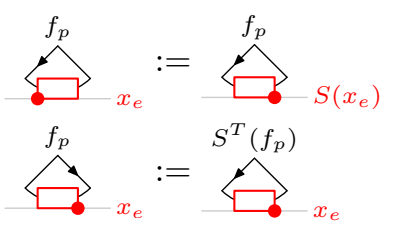

where all these elementary diagrams are assumed to be invariant under arbitrary rotations, for instance:

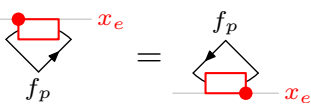


Note also that $S^{T}=\left(S^{-1}\right)^{T}$ is the correct antipode of $X=\left(H^{\mathrm{op}}\right)^{*}$. As a consequence, any of the above elementary diagrams has the same value as its mirror image under reflection about a vertical axis. In fact, 140 and 141 are mirror images of each other in that sense. Furthermore for some $f_{p}$ (such as the dual Haar integral $\phi$ ) it may happen that $S^{T}\left(f_{p}\right)=f_{p}$ and the loop orientation may become unimportant.

If the face $p$ has edges other than $e$ in its boundary we may extend the above diagrams as follows. Pick another edge $e^{\prime} \in E(p)$ which shares a common vertex with $e$. Then for any $x_{e^{\prime}} \in H$ we define a "virtual" glueing operation by

$$
\sum_{\left(f_{p}\right)} x_{x_{e}}^{\prime \prime}=f_{p}\left(x_{e^{\prime}} x_{e}\right)
$$

where the arrows indicate the order in which the coproduct of $f_{p}$ is applied to the elementary diagrams. The black dot denotes the origin for this comultiplication. While this origin is very important in general it can be neglected iff $f_{p}$ is cocommutative. In that case we will simply omit the corresponding dot from the diagram as we did implicitly in Definition 2. In any case one needs to pay attention to the correct comultiplication in $X$ which causes the product around the edges of $p$ to be taken in clockwise order.

Finally for any interior edge $e \in E_{M}$ 。 with adjacent interior or boundary faces $p, q \in F \cup F_{\partial M}$ we pick $x_{e} \in H$ and $f_{p}, f_{q} \in X$ arbitrarily and define a "physical" glueing operation by

$$
\sum_{f_{p^{\prime}}}:=\sum_{\left(x_{e}\right)}
$$

where the order of comultiplication is determined by the orientation of the underlying graph edge. Consequently one has for instance

$$
\sum_{f_{p^{\prime}}}^{\sum_{f_{p^{\prime}}}} x_{x_{e}} \sum_{x_{e}}{ }_{x_{e}^{\prime \prime}}=\sum_{\left(x_{e}\right)}
$$

which is perfectly compatible with 116 as expected. In contrast to elementary diagrams such a composite diagram may only be invariant under rotation by $\pi$ 


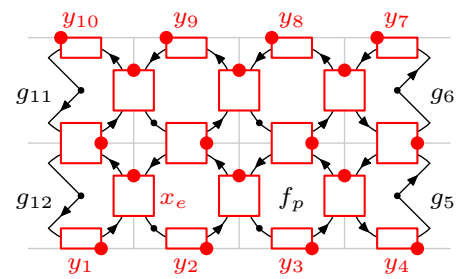

Fig. 5. Diagram encoding the tensor trace $\operatorname{ttr}\left(\left\{x_{e}\right\} ;\left\{f_{p}\right\} ;\left\{y_{e}\right\} ;\left\{g_{q}\right\}\right)$. While the interior degrees of freedom $\left\{x_{e}\right\} \subset H$ and $\left\{f_{p}\right\} \subset X$ are only shown partially the boundary degrees of freedom $\left\{y_{e}\right\} \subset H$ and $\left\{g_{q}\right\} \subset X$ are labelled in such a way that the ordering of the boundary is evident.

if $S\left(x_{e}\right)=x_{e}$. Also note that in general a simultaneous reversal of both virtual loops is not given by reflecting a composite diagram about its vertical axis:

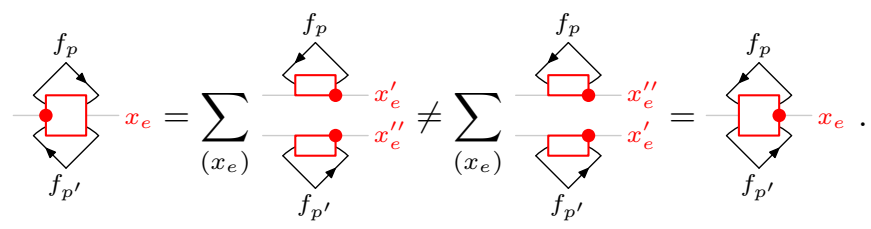

Rather, equality holds iff $x_{e} \in \operatorname{Cocom}(H)$. This should be compared with (7).

Thus by starting from the elementary diagram $(139)$ we have reexpressed the evaluation rule (136) for an interior edge entirely in terms of "virtual" (143) and "physical" (144) glueing operations. These are given by comultiplication in $X$ and $H$ respectively. This means that an interior edge is formed by appropriately glueing two boundary edges together via comultiplication. Alternatively, one may regard this as glueing together two virtual interior loops. Virtual loops themselves are assembled via glueing together smaller loop pieces.

In summary we have the following general

Definition 3 (Hopf tensor trace). Let $E_{M}$ and $F_{M}$ the sets of interior edges and faces respectively, and accordingly $E_{\partial M}$ and $F_{\partial M}$ the sets of boundary edges and faces.

The Hopf tensor trace associated with the graph $\Gamma$ is the function $\operatorname{ttr}_{\Gamma}$ : $H^{\otimes\left|E_{M^{0}}\right|} \otimes X^{\otimes\left|F_{M}\right|} \otimes H^{\otimes\left|E_{\partial M}\right|} \otimes X^{\otimes\left|F_{\partial M}\right|} \rightarrow \mathbb{C}$,

$$
\bigotimes_{e \in E_{M^{\circ}}} x_{e} \bigotimes_{p \in F_{M}} f_{p} \bigotimes_{e \in E_{\partial M}} y_{e} \bigotimes_{q \in F_{\partial M}} g_{q} \mapsto \operatorname{ttr}_{\Gamma}\left(\left\{x_{e}\right\} ;\left\{f_{p}\right\} ;\left\{y_{e}\right\} ;\left\{g_{q}\right\}\right)
$$

which is defined via diagrams and the evaluation rules 139, 140, 1141, 143) and (144).

Note that this generalized Hopf tensor trace reduces to the Hopf tensor trace for a surface without boundary (as in Definition 2 in the natural way by restricting any virtual loop function $f_{p}$ to be cocommutative and setting:

$$
\operatorname{ttr}_{\Gamma}\left(\left\{x_{e}\right\} ;\left\{f_{p}\right\}\right):=\operatorname{ttr}_{\Gamma}\left(\left\{x_{e}\right\} ;\left\{f_{p}\right\} ; \emptyset ; \emptyset\right) .
$$


4.2. Quantum states. So far we have merely defined a particular, fully contracted tensor network (which is a complex number) with no reference to a quantum many-body state whatsoever. We now take the next step and use the tensor trace above to generate actual quantum states in a remarkably straightforward fashion:

Definition 4 (Hopf tensor network state). Let $x_{e}, y_{e} \in H$ and $f_{p}, g_{q} \in X$ as in Definition 3. Let $\Gamma$ the graph embedded in the surface $M$.

1. If $\partial M \neq \emptyset$ then

$$
\begin{aligned}
& \left|\psi_{\Gamma}\left(\left\{x_{e}\right\} ;\left\{f_{p}\right\} ;\left\{y_{e}\right\} ;\left\{g_{q}\right\}\right)\right\rangle \\
& \quad:=\sum_{\substack{\left(x_{e}\right) \\
e \in E_{M^{\circ}}}} \sum_{\substack{\left(y_{e}\right) \\
e \in E_{\partial M}}} \operatorname{ttr}_{\Gamma}\left(\left\{x_{e}^{\prime \prime}\right\} ;\left\{f_{p}\right\} ;\left\{y_{e}^{\prime \prime}\right\} ;\left\{g_{q}\right\}\right) \bigotimes_{e \in E_{M^{\circ}}}\left|x_{e}^{\prime}\right\rangle \bigotimes_{e \in E_{\partial M}}\left|y_{e}^{\prime}\right\rangle .
\end{aligned}
$$

2. If $\partial M=\emptyset$ then

$$
\left|\psi_{\Gamma}\left(\left\{x_{e}\right\} ;\left\{f_{p}\right\}\right)\right\rangle:=\sum_{\substack{\left(x_{e}\right) \\ e \in E_{M}}} \operatorname{ttr}_{\Gamma}\left(\left\{x_{e}^{\prime \prime}\right\} ;\left\{f_{p}\right\}\right) \bigotimes_{e \in E_{M}}\left|x_{e}^{\prime}\right\rangle
$$

In both cases we call the resulting state a Hopf tensor network state on the graph $\Gamma$.

Given an arbitrary region $R$ within the surface $M$ it is straightforward to partition such a quantum state into interior and exterior parts accordingly. In the language of Hopf tensor network states this simply amounts to cutting virtual loops. Without loss of generality we will show this for a graph embedded in a surface without boundaries.

Before proceeding we need to clarify though what a region $R$ of a surface discretized by the graph $\Gamma$ is supposed to mean. We will only consider regions whose boundary does not cross any edge of $\Gamma$. Equivalently, such a boundary may only run through vertices and faces or along edges of $\Gamma$. Any part of the boundary which coincides with a graph edge will be called smooth and otherwise rough. This coincides with the way a graph may be embedded in a surface with boundary (see Figure 4). It is clear that a valid region $R$ is determined by a subgraph $\Gamma_{R}$ up to deformations of rough boundaries, hence we will define a region by its associated subgraph. This means that a partition of $\mathrm{M}$ into $R$ and its complement $\bar{R}$ naturally divides $E$ into the disjoint sets $E_{R}$ and $E_{\bar{R}}$. At the same time it divides $F$ into the disjoint sets $F_{R}, F_{\bar{R}}$ and $F_{\partial R}$ or, put differently, boundary faces $F_{\partial R}$ are created which do not belong to either subgraph $\Gamma_{R}$ or $\Gamma_{\bar{R}}$. In contrast, there are no boundary edges. Hence the (discretized) boundary of the region $R$ coincides with $F_{\partial R}$ and has a length of $\left|F_{\partial R}\right|$. By abuse of notation we will call this length simply $|\partial R|$.

Proposition 2 (Partitions). Let $\left|\psi_{\Gamma}\left(\left\{x_{e}\right\} ;\left\{f_{p}\right\}\right)\right\rangle$ be a Hopf tensor network state on the graph $\Gamma$ and $R$ an arbitrary region which is associated to the subgraph $\Gamma_{R}=\left(V_{R}, E_{R}, F_{R}\right) \subset \Gamma$. Let $g_{q} \in X$ and define

$$
\left|\psi_{R}\left(\left\{g_{q}\right\}\right)\right\rangle:=\left|\psi_{\Gamma_{R}}\left(\left\{x_{e}\right\}_{R} ;\left\{f_{p}\right\}_{R} ; \emptyset ;\left\{g_{q}\right\}\right)\right\rangle
$$


where $\left\{x_{e}\right\}_{R}=\left\{x_{e} \mid e \in E_{R}\right\}$ and $\left\{f_{p}\right\}_{R}=\left\{f_{p} \mid p \in F_{R}\right\}$ are the natural restrictions to $\Gamma_{R}$. Then

$$
\left|\psi_{\Gamma}\left(\left\{x_{e}\right\} ;\left\{f_{p}\right\}\right)\right\rangle=\sum_{\substack{\left(f_{q}\right) \\ q \in F_{\partial R}}}\left|\psi_{R}\left(\left\{f_{q}^{\prime}\right\}\right)\right\rangle \otimes\left|\psi_{\bar{R}}\left(\left\{f_{q}^{\prime \prime}\right\}\right)\right\rangle .
$$

Proof. Obvious from the complete diagrams and evaluation rule 143 which needs to be applied backwards.

Building on this general framework of Hopf tensor network states we can solve the generalized quantum double model now. Namely, we identify a particular Hopf tensor network state as a ground state of the model. As such this state is directly seen to be topologically ordered. Note that we will only make use of the structure maps of $H$ as well as the Haar integral and its dual to describe the state. Again we assume a surface without boundaries.

Theorem 3 (Ground state of the generalized quantum double model). Let $h \in H$ and $\phi \in X$ the respective Haar integrals. The state

$$
\left|\psi_{\Gamma}\right\rangle:=\left|\psi_{\Gamma}(h, \ldots, h ; \phi, \ldots, \phi)\right\rangle
$$

is a ground state of the $\mathrm{D}(H)$-model.

Proof. Since the Hamiltonian of the $\mathrm{D}(H)$-model is a sum of local, commuting terms by Theorem 2 it is enough to show that each operator $A(s)$ and $B(p)$ leaves the state $\left|\psi_{\Gamma}\right\rangle$ invariant individually. In order to do so we may partition $\left|\psi_{\Gamma}\right\rangle$ into an interior part corresponding to the support of such an operator and an exterior part. Both parts are glued via comultiplication in $X$. It will then suffice to prove that this interior part remains unchanged by either $A(s)$ or $B(p)$ respectively.

Hence consider a face $p \in F$ with a boundary consisting of $r$ edges. The interior part of $\left|\psi_{\Gamma}\right\rangle$ is given by

$$
\begin{aligned}
\left|\psi_{p}\left(f_{1}, \ldots, f_{r}\right)\right\rangle & =\sum_{\left(h_{i}\right)}{ } \sum_{r} \phi\left(h_{r}^{\prime \prime \prime} \cdots h_{1}^{\prime \prime \prime}\right) \prod_{j=1}^{r} f_{j}\left(S\left(h_{j}^{\prime \prime}\right)\right)\left|h_{1}^{\prime}\right\rangle \otimes \cdots \otimes\left|h_{r}^{\prime}\right\rangle .
\end{aligned}
$$

with the Haar integrals $h_{i}=h \in H$ and arbitrary $f_{i} \in X$. It is invariant under the action of $B(p)$ as can be seen from

$$
\begin{aligned}
& B(p)\left|\psi_{p}\left(f_{1}, \ldots, f_{r}\right)\right\rangle \\
& =\sum_{\left(h_{i}\right)} \phi\left(h_{r}^{(4)} \cdots h_{1}^{(4)}\right) \phi\left(h_{r}^{(1)} \cdots h_{1}^{(1)}\right) \prod_{j=1}^{r} f_{j}\left(S\left(h_{j}^{(3)}\right)\right)\left|h_{1}^{(2)}\right\rangle \otimes \cdots \otimes\left|h_{r}^{(2)}\right\rangle
\end{aligned}
$$




$$
\begin{aligned}
& =\sum_{\left(h_{i}\right)} \phi\left(h_{r}^{(3)} \cdots h_{1}^{(3)}\right) \phi\left(h_{r}^{(4)} \cdots h_{1}^{(4)}\right) \prod_{j=1}^{r} f_{j}\left(S\left(h_{j}^{(2)}\right)\right)\left|h_{1}^{(1)}\right\rangle \otimes \cdots \otimes\left|h_{r}^{(1)}\right\rangle \\
& =\sum_{\left(h_{i}\right)} \phi^{2}\left(h_{r}^{\prime \prime \prime} \cdots h_{1}^{\prime \prime \prime}\right) \prod_{j=1}^{r} f_{j}\left(S\left(h_{j}^{\prime \prime}\right)\right)\left|h_{1}^{\prime}\right\rangle \otimes \cdots \otimes\left|h_{r}^{\prime}\right\rangle \\
& =\left|\psi_{p}\left(f_{1}, \ldots, f_{r}\right)\right\rangle .
\end{aligned}
$$

Next consider a vertex $s \in V$ with $r$ attached edges. In this case the interior part of $\left|\psi_{\Gamma}\right\rangle$ reads

$$
\begin{aligned}
\left|\psi_{s}\left(f_{1}, \ldots, f_{r}\right)\right\rangle= & =\sum_{\left(h_{i}\right)} \prod_{j=1}^{r} f_{j}\left(S\left(h_{j}^{\prime \prime}\right) h_{j+1}^{\prime \prime \prime}\right)\left|h_{1}^{\prime}\right\rangle \otimes \cdots \otimes\left|h_{r}^{\prime}\right\rangle .
\end{aligned}
$$

Note that the orientation of the graph edges can always be reduced to the above setting using 116 for kets and (140) for diagrams. Now we obtain

$$
\begin{aligned}
A(s)\left|\psi_{s}\left(f_{1}, \ldots, f_{r}\right)\right\rangle & \\
= & \sum_{\left(h_{i}\right)} \prod_{j=2}^{r-1} f_{j}\left(S\left(h_{j}^{\prime \prime}\right) h_{j+1}^{\prime \prime \prime}\right) \sum_{(h)} f_{1}\left(S\left(h_{1}^{\prime \prime}\right) h_{2}^{\prime \prime \prime}\right) f_{r}\left(S\left(h_{r}^{\prime \prime}\right) h_{1}^{\prime \prime \prime}\right) \\
& \left|h^{(1)} h_{1}^{\prime}\right\rangle \otimes \cdots \otimes\left|h^{(r)} h_{r}^{\prime}\right\rangle \\
= & \sum_{\left(h_{i}\right)} \prod_{j=3}^{r-1} f_{j}\left(S\left(h_{j}^{\prime \prime}\right) h_{j+1}^{\prime \prime \prime}\right) \sum_{(h)} f_{1}\left(S\left(h_{1}^{\prime \prime}\right) h^{(2)} h_{2}^{\prime \prime \prime}\right) f_{2}\left(S\left(h_{2}^{\prime \prime}\right) h_{3}^{\prime \prime \prime}\right) f_{r}\left(S\left(h^{(1)} h_{r}^{\prime \prime}\right) h_{1}^{\prime \prime \prime}\right) \\
& \left|h_{1}^{\prime}\right\rangle \otimes\left|h^{(3)} h_{2}^{\prime}\right\rangle \otimes \cdots \otimes\left|h^{(r+1)} h_{r}^{\prime}\right\rangle \\
= & \sum_{\left(h_{i}\right)} f_{1}\left(S\left(h_{1}^{\prime \prime}\right) h_{2}^{\prime \prime \prime}\right) \prod_{j=3}^{r-1} f_{j}\left(S\left(h_{j}^{\prime \prime}\right) h_{j+1}^{\prime \prime \prime}\right) \sum_{(h)} f_{2}\left(S\left(h_{2}^{\prime \prime}\right) h^{(2)} h_{3}^{\prime \prime \prime}\right) f_{r}\left(S\left(h^{(1)} h_{r}^{\prime \prime}\right) h_{1}^{\prime \prime \prime}\right) \\
& \left|h_{1}^{\prime}\right\rangle \otimes\left|h_{2}^{\prime}\right\rangle \otimes\left|h^{(3)} h_{3}^{\prime}\right\rangle \otimes \cdots \otimes\left|h^{(r)} h_{r}^{\prime}\right\rangle \\
= & \sum_{\left(h_{i}\right)} \prod_{j=1}^{r-2} f_{j}\left(S\left(h_{j}^{\prime \prime}\right) h_{j+1}^{\prime \prime \prime}\right) \sum_{(h)} f_{r-1}\left(S\left(h_{r-1}^{\prime \prime}\right) h^{(2)} h_{r}^{\prime \prime \prime}\right) f_{r}\left(S\left(h_{1}^{(1)} h_{r}^{\prime \prime}\right) h_{1}^{\prime \prime \prime}\right) \\
& \left|h_{1}^{\prime}\right\rangle \otimes \cdots \otimes\left|h_{r-1}^{\prime}\right\rangle \otimes\left|h^{(3)} h_{r}^{\prime}\right\rangle \\
= & \left|\psi_{s}\left(f_{1}, \ldots, f_{r}\right)\right\rangle
\end{aligned}
$$

where we repeatedly used Lemma 4. This concludes the proof. 
In fact, the proof of Theorem 3 implies that $\left|\psi_{\Gamma}\right\rangle$ is also invariant under each local action of $\mathrm{D}(H)$ at any site $(s, p)$, not just under the operators constituting the Hamiltonian. More precisely, one has

$$
B_{f}(s, p) A_{a}(s, p)\left|\psi_{\Gamma}\right\rangle=\epsilon(a) f\left(1_{H}\right)\left|\psi_{\Gamma}\right\rangle
$$

for any $f \otimes a \in \mathrm{D}(H)$. This can be easily seen from the local $\mathrm{D}(H)$-module structure, the fact that $A(s)=A_{h}(s, p)$ and $B(p)=B_{\phi}(s, p)$ leave $\left|\psi_{\Gamma}\right\rangle$ strictly invariant and the properties of the Haar integrals. In other words, the quantum state $\left|\psi_{\Gamma}\right\rangle$ is nothing but a trivial representation of the quantum double $\mathrm{D}(H)$. Comparing with the comment after Theorem 2 one realizes that $\left|\psi_{\Gamma}\right\rangle$ should be viewed as the spatially distributed version of the integral $\phi \otimes h \in \mathrm{D}(H)$. For these reasons one may call $\left|\psi_{\Gamma}\right\rangle$ the vacuum of the model and as such it has trivial topological charge everywhere.

Let us emphasize again that one really needs just a single datum, i.e. the finite-dimensional Hopf $C^{*}$-algebra $H$, to produce this topological ground state since the Haar integral $h$ (and the Haar functional $\phi$ ) is uniquely defined. In particular, the construction is fully basis-independent.

Furthermore the construction is symmetric ${ }^{7}$ in the algebras $H$ and $X$ with their respective integrals $h$ and $\phi$, hence it has a natural dual notion. In fact, this foreshadows electric-magnetic duality as shown in 12 .

While we excluded surface boundaries explicitly for Theorem 3 the following example shows what one can learn from the presence of boundaries imposed by the underlying surface.

Example 1. Consider the graph $\Gamma$ underlying the diagram shown in Figure 5 and let $H=\mathbb{C Z}_{2}$. Then the state

$$
\left|\psi_{0}\right\rangle:=\left|\psi_{\Gamma}(\{h\} ;\{\phi\} ;\{h\} ;\{\phi\})\right\rangle
$$

is a codeword of the surface code defined in [9]. It encodes the logical state $|+\rangle$. The other codeword $\left|\psi_{1}\right\rangle$ can be obtained by acting on this Hopf tensor network state with the appropriate string operator connecting the two rough boundaries. This generalizes to any finite-dimensional Hopf $C^{*}$-algebra by using the appropriate ribbon operators.

Remark 1. From the proof of Theorem 3 it is clear that any Hopf tensor network state of the form

$$
\left|\psi_{\Gamma}\left(h, \ldots, h ; f_{1}, \ldots, f_{|F|}\right)\right\rangle
$$

is invariant under all vertex operators $A(s)$. From the perspective of lattice gauge theory this means that deforming the ground state $\left|\psi_{\Gamma}\right\rangle$ of the $\mathrm{D}(H)$-model by changing the functions $\{\phi\} \mapsto\left\{f_{i}\right\}$ only will never break the gauge symmetry. These deformations in the state might therefore well correspond to a local perturbation of the Hamiltonian and thus preserve topological order provided the strength of the perturbation is limited to a certain finite threshold [8, 7]. Some initial work towards this direction has been conducted in 14] which is concerned with tensor network deformations of the toric code.

\footnotetext{
7 Strictly speaking, symmmetry holds up to a flip in the comultiplication, which is precisely the difference between $H^{*}$ and $X$.
} 
4.3. Hierarchy. Apart from ground states of the $\mathrm{D}(H)$-models the framework of Hopf tensor network states based on an arbitrary Hopf $C^{*}$-algebra $H$ comprises more intriguing examples of quantum many-body states. These can be obtained from certain other choices of elements in $H$ and $X$ which are motivated both by the algebraic structure itself as well as by ideas of charge condensation [4,5]:

Definition 5 (Hierarchy). Let $A \subset H$ and $B \subset X$ Hopf subalgebras with Haar integrals $h_{A} \in A$ and $\phi_{B} \in B$ respectively. Set

$$
\left|\psi_{\Gamma}^{A, B}\right\rangle:=\left|\psi_{\Gamma}\left(h_{A}, \ldots, h_{A} ; \phi_{B}, \ldots, \phi_{B}\right)\right\rangle .
$$

Obviously, with the choice $A=H$ and $B=X$ we recover the state $\left|\psi_{\Gamma}\right\rangle$ which is topologically ordered as a ground state of the $\mathrm{D}(H)$-model. On the other hand, if $B=\left\{1_{X}\right\}$ is the trivial Hopf subalgebra of $X$ then the resulting Hopf tensor network state is a product state for any Hopf subalgebra $A \subset H$. This can be seen from (23). Indeed, suppose a face $p$ has $r$ edges in its boundary and $f_{p}=1_{X}$. Then iterating (23) yields

$$
\sum_{\left(f_{p}\right)} f_{p}^{(1)} \otimes \cdots \otimes f_{p}^{(r)}=1_{X} \otimes \cdots \otimes 1_{X}
$$

and consequently one has

$$
\operatorname{ttr}_{\Gamma}\left(\left\{x_{e}\right\} ; 1_{X}, \ldots, 1_{X}\right)=\prod_{e \in E_{M}} \overbrace{1_{X}}^{1_{X}} x_{e}
$$

up to a possible application of the antipode $S$ on each graph edge depending on its orientation. Since $1_{X}=\epsilon^{T}\left(1_{\mathbb{C}}\right)$ is the analogue of $\sqrt{18}$ for the Hopf $C^{*}$ algebra $X$ we deduce

$$
\bigodot_{1_{X}}^{1_{X}} x_{e}=\sum_{\left(x_{e}\right)}\left\langle\epsilon^{T}\left(1_{\mathbb{C}}\right), S\left(x_{e}^{\prime}\right)\right\rangle\left\langle\epsilon^{T}\left(1_{\mathbb{C}}\right), x_{e}^{\prime \prime}\right\rangle=\sum_{\left(x_{e}\right)} \epsilon\left(S\left(x_{e}^{\prime}\right)\right) \epsilon\left(x_{e}^{\prime \prime}\right)=\epsilon\left(x_{e}\right)
$$

and therefore

$$
\operatorname{ttr}_{\Gamma}\left(\left\{x_{e}\right\} ; 1_{X}, \ldots, 1_{X}\right)=\prod_{e \in E_{M}} \epsilon\left(x_{e}\right)
$$

which no longer depends on the orientation of $\Gamma$. Now by virtue of 150 the quantum state

$$
\left|\psi_{\Gamma}\left(h_{A}, \ldots, h_{A} ; 1_{X}, \ldots, 1_{X}\right)\right\rangle=\sum_{\left(h_{A, e}\right)} \bigotimes_{e \in E_{M}} \epsilon\left(h_{A, e}^{\prime \prime}\right)\left|h_{A, e}^{\prime}\right\rangle=\bigotimes_{e \in E_{M}}\left|h_{A}\right\rangle
$$

is seen to factor into a simple product state.

In between these two extremes a hierarchy of quantum states unfolds which are indexed by different choices of $A$ and $B$. However, depending on the Hopf algebra $H$ in question the interior of this hierarchy may collapse partially. This means that different pairs $(A, B)$ of Hopf subalgebras may actually define identical quantum states. Unfortunately we do not know how to characterize the 
surviving equivalence classes of states in closed form without additional assumptions on $H$.

However, if $H=\mathbb{C} G$ we have a clear picture of the above hierarchy. It turns out that the classes of Hopf tensor network states emerging from the partial collapse are isomorphic to ground states of certain quantum double models based on groups smaller than $G$. Indeed, the relevant Hopf subalgebras in this case exactly read $A=\mathbb{C} K$ and $B=\mathbb{C}^{G / N}$ where $K \subset G$ is a subgroup and $N \triangleleft$ $G$ a normal subgroup (see Section 2.4.3). For simplicity we abbreviate such a pair of Hopf subalgebras by $(K, N)$. Then it is not difficult to see that both $(K, N)$ and $(K, K \cap N)$ yield identical Hopf tensor network states. Furthermore, if $\left|k_{1}, \ldots, k_{|E|}\right\rangle$ with $k_{e} \in K$ for each edge $e \in E$ is a basis state then one has

$$
\left\langle k_{1}, \ldots, k_{|E|} \mid \psi_{\Gamma}^{K, K \cap N}\right\rangle=\left\langle k_{1} l_{1}, \ldots, k_{|E|} l_{|E|} \mid \psi_{\Gamma}^{K, K \cap N}\right\rangle
$$

for arbitrary elements $l_{e} \in K \cap N$, so all amplitudes are actually constant on the cosets $k_{e}(K \cap N)$. This means that one may apply the canonical projection $\pi: K \rightarrow K /(K \cap N)$ at each edge and regard $\phi_{K \cap N}$ as the Haar integral of $\mathbb{C}^{K /(K \cap N)}$. Hence the resulting state coincides with the ground state $\left|\psi_{\Gamma}\right\rangle$ of the quantum double based on the group algebra of the group $K /(K \cap N) \simeq K N / N$.

We conclude that the hierarchy of Hopf tensor network states arranges quantum double models based on different groups in one coherent picture. These groups are precisely isomorphic to $K N / N$ so the equivalence classes of states in the hierarchy are the trivial representations of the quantum doubles $\mathrm{D}(\mathbb{C}(K N / N))$. These comprise all possibilities for the residual symmetry algebra after the full $\mathrm{D}(\mathbb{C} G)$-symmetry has been partially broken down to a smaller symmetry algebra [4,5]. For that reason the quantum states in our hierarchy realize the condensation of topological charges within the same underlying Hilbert space.

4.4. PEPS. At this stage it is important to make contact with one of the usual formulations of tensor network states. In the PEPS approach 43 a quantum state is represented by choosing some fixed basis and encoding the wavefunction amplitude for each basis element in a fully contracted tensor network. Contrastingly, our Hopf tensor network states are defined without reference to any basis. It is rather the choice of particular, often canonical, elements $\left\{x_{e}\right\} \subset H$ and $\left\{f_{p}\right\} \subset X$ which determines the properties of the quantum state. If the need arises one can still obtain an explicit wavefunction expansion in a straightforward manner. As the next example shows, those distinguished elements naturally generate all relevant sums and amplitudes.

Example 2. Let $H=\mathbb{C}^{G}$. We would like to explicitly construct the Hopf tensor network state $\left|\psi_{\Gamma}\right\rangle$ for the graph $\Gamma$ shown in Figure 6 and obtain an expansion in terms of the canonical basis $\left\{\delta_{g} \mid g \in G\right\}$ of $\mathbb{C}^{G}$. We assume the underlying surface to be homeomorphic to $S^{2}$ as indicated in Figure 6 . Using the Haar integral $h_{i}=\delta_{e}$ of the dual group algebra $\mathbb{C}^{G}$ and its corresponding Haar functional 


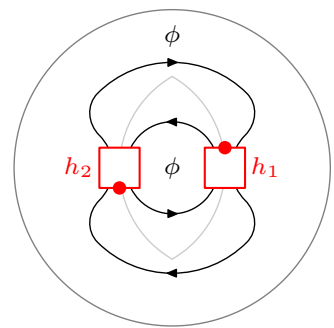

Fig. 6. Hopf tensor network state $\left|\psi_{\Gamma}\right\rangle$ on a small graph $\Gamma$ embedded in $S^{2}$. The outer circle is identified with the north pole of $S^{2}$.

$\phi \in H$ we get by Theorem 3

$$
\begin{aligned}
\left|\psi_{\Gamma}\right\rangle & =\sum_{\left(h_{i}\right)} \phi\left(h_{1}^{\prime \prime \prime} h_{2}^{\prime \prime \prime}\right) \phi\left(S\left(h_{1}^{\prime \prime}\right) S\left(h_{2}^{\prime \prime}\right)\right)\left|h_{1}^{\prime}\right\rangle \otimes\left|h_{2}^{\prime}\right\rangle \\
& =\sum_{\left(h_{i}\right)}\left\langle\phi \otimes \phi, \Delta\left(h_{1}^{\prime \prime} h_{2}^{\prime \prime}\right)\right\rangle\left|h_{1}^{\prime}\right\rangle \otimes\left|h_{2}^{\prime}\right\rangle \\
& =\sum_{\left(h_{i}\right)} \phi\left(h_{1}^{\prime \prime} h_{2}^{\prime \prime}\right)\left|h_{1}^{\prime}\right\rangle \otimes\left|h_{2}^{\prime}\right\rangle \\
& =\sum_{u, v \in G} \phi\left(\delta_{u^{-1}} \delta_{v^{-1}}\right)\left|\delta_{u}\right\rangle \otimes\left|\delta_{v}\right\rangle \\
& =\frac{1}{|G|} \sum_{g \in G}\left|\delta_{g}\right\rangle \otimes\left|\delta_{g}\right\rangle .
\end{aligned}
$$

Interestingly, while the Haar integral $\delta_{e}$ merely represents a single element of the basis, it is actually the act of comultiplication which produces the sum over the entire basis from $\delta_{e}$.

As outlined in the introduction PEPS are defined in terms of local tensors with open virtual indices, for a particular example see (4). Contrastingly, our diagrammatic notation as given by (143) uses the comultiplication of the Hopf algebra to disconnect and separate local objects on the virtual level, hence it appears there are no virtual indices at all in our formalism.

This is not entirely true. If the functions $\left\{f_{p}\right\}$ for evaluating virtual loops belong to a particular class 8 then indeed one may regard the evaluation of a virtual loop as tracing over a product of certain matrices, once a particular basis of $H$ has been chosen. Consequently, each such matrix naturally constitutes part of a local tensor with open virtual indices and evaluating the loop corresponds to a cyclic contraction of those indices. Note that this applies to all Hopf tensor network states of our hierarchy (but is not limited to these). For example, for the state $\left|\psi_{\Gamma}\right\rangle$ at the top of the hierarchy one has the local projector

$$
P=\|h\|^{-1} \sum_{(h)} \sum_{\alpha, \beta, \gamma, \delta \in \mathcal{B}}\left(L_{+}^{S\left(h^{\prime \prime}\right)}\right)_{\alpha \beta}\left(L_{+}^{h^{\prime \prime \prime}}\right)_{\gamma \delta}\left|h^{\prime}\right\rangle\langle\alpha, \beta, \gamma, \delta|
$$

${ }^{8}$ This is the so-called character ring $R_{\mathbb{C}}(H)=\sum_{i=1}^{n} \mathbb{C} \chi_{i}$ where the $\chi_{i}$ are the irreducible characters of $H$. 
where $\left(L_{+}^{a}\right)_{\alpha \beta}$ denotes a matrix element of the action 102 with respect to some basis $\mathcal{B}{ }^{9}$ Note that in this setting the local matrices encode both information about the spin state on the graph edge as well as about the function used to evaluate the virtual loop.

Furthermore, if a Hopf tensor trace admits a representation in terms of local tensors with open indices properties like injectivity may be studied. More precisely, a PEPS is called injective [39] if there is a partition of the underlying graph in disjoint regions $R_{i}$ such that for every region $R_{i}$ the linear map from open virtual indices at the boundary to physical indices in the interior is injective. Whether or not a particular tensor network representation of a quantum state is injective has important consequences for e.g. the existence of a parent Hamiltonian that has the given PEPS as its unique ground state 39. One can show that all Hopf tensor network states $\left|\psi_{\Gamma}^{A, B}\right\rangle$ in the hierarchy are not injective for any finite-dimensional Hopf $C^{*}$-algebra $H$. However, (at least) the state $\left|\psi_{\Gamma}\right\rangle$ at the top of the hierarchy obeys a relaxed version of injectivity which one might call $\mathrm{H}$-injectivity. This is a certain generalization of the $G$-injectivity condition defined in 41.

\section{Calculating the topological entanglement entropy}

Topological order is commonly associated with non-local order parameters. It is believed that among these resides the topological entanglement entropy $\gamma$ [27. 32 which is a universal additive correction to the area law for the entanglement entropy of a bipartition of the system into a region $R$ and its complement. Given a ground state of a topologically ordered system, the entanglement entropy $S_{R}$ for a region $R$ is argued to scale as

$$
S_{R}=\alpha \cdot|\partial R|-\gamma
$$

in the limit of infinitely large regions. Here $\alpha$ is a parameter which encodes non-universal behaviour on short length scales. In fact, the topological entanglement entropy $\gamma$ also contains partial information about the types of quasiparticle excitations that may occur in the low-energy sector of the system.

For these reasons we would like to show in this section how the non-local order parameter $\gamma$ can be understood naturally in the context of Hopf tensor network states. In particular, we are going to show that different classes of finitedimensional Hopf $C^{*}$-algebras yield fundamentally different mechanisms for the emergence of a non-vanishing topological entanglement entropy.

To this end, we will exploit the generic decomposition of Hopf tensor network states into interior and exterior parts as stated in Proposition 2, We will then compute the block entropy directly from certain properties of the reduced density operator. In order to render its simple structure evident we will make use of isometries and completely clear out the interior of both the region $R$ and its complement $\bar{R}$. Effectively, we concentrate all topological information contained in the ground state into the (inner) boundaries of the system. Furthermore, the distillation process will be crafted such that all intermediate quantum states can be kept track of conveniently via Hopf tensor traces.

\footnotetext{
9 Furthermore one may express the (physical) ket in the same basis, too, and appreciate the similarities and differences as compared to the trivial case 10 .
} 
It should be noted that our scheme of applying isometries implements entanglement renormalisation [45, 46] both for the $\mathrm{D}(H)$-models as well as for states of the hierarchy. In fact, it directly extends the work in [1] which is concerned with states at the top of the hierarchy for $H=\mathbb{C} G$. At the same time our scheme provides a complementary view on entanglement renormalisation for string-net models [30, namely from the perspective of a local symmetry algebra.

5.1. Isometries. We begin by developing the distillation process. For that we will introduce a hierarchy of "little" isometries that insert minimal faces and vertices into a given graph. The little isometries at the top end of the hierarchy are taylored to the generalized quantum double model, i.e. they preserve all excitations of that model. Subsequently we will define a pair of local unitary maps that allow for reconnecting edges. Taken together, both the little isometries and the unitary maps will yield a hierarchy of isometries that add or remove arbitrary edges. Their effect on Hopf tensor network states will be analyzed in the next section.

As far as notation is concerned we will always denote the original graph by $\Gamma_{1}$ and the modified one by $\Gamma_{2}$. Whenever it is appropriate to talk about actual quantum double models, $\mathcal{H}_{i}$ will denote the Hamiltonian of the $\mathrm{D}(H)$-model on the graph $\Gamma_{i}$.

Proposition 3 (Little isometries). Let $A \subset H$ and $B \subset X$ Hopf subalgebras with the respective Haar integrals $h_{A} \in A$ and $\phi_{B} \in B$. Additionally, let

$$
\begin{aligned}
\lambda_{A, B} & :=\sum_{\left(h_{A}\right)} \phi_{B}\left(h_{A}^{\prime \prime}\right) h_{A}^{\prime}, \\
1_{A, B} & :=\frac{\lambda_{A, B}}{\left\|\lambda_{A, B}\right\|}, \\
\Lambda_{A} & :=\frac{h_{A}}{\left\|h_{A}\right\|}=\frac{1}{\sqrt{\phi\left(h_{A}\right)}} h_{A} .
\end{aligned}
$$

Then the linear maps $i_{F}^{A, B}$ and $i_{V}^{A}$ defined via

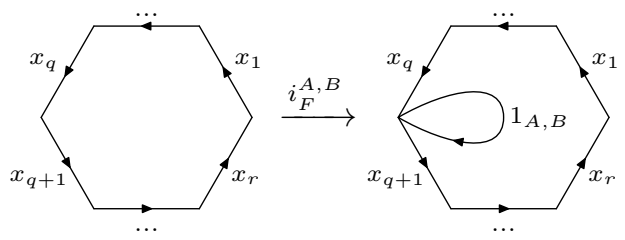

and

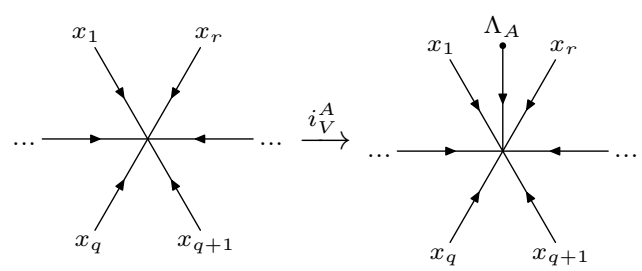


are isometries.

Furthermore, for $i_{F}:=i_{F}^{H, H^{*}}$ and $i_{V}:=i_{V}^{H}$ one has

$$
\begin{aligned}
& i_{F} \mathcal{H}_{1}=\mathcal{H}_{2} i_{F}, \\
& i_{V} \mathcal{H}_{1}=\mathcal{H}_{2} i_{V} .
\end{aligned}
$$

Proof. For the first claim, it is enough to show the invariance of the inner product or equivalently that $\left\|1_{A, B}\right\|=\left\|\Lambda_{A}\right\|=1$. Indeed, from

$$
\left\|h_{A}\right\|^{2}=\left(h_{A}, h_{A}\right)=\phi\left(h_{A}^{*} h_{A}\right)=\phi\left(h_{A}^{2}\right)=\phi\left(h_{A}\right) .
$$

this is easily seen to be true.

As for the second claim we note that $i_{F}$ inserts a loop which is automatically stabilized by the corresponding face operator in $\mathcal{H}_{2}$. It is also easily seen that $1_{H, H^{*}}=1_{H}$ and hence it does not affect the magnetic flux through the original face as measured by either $\mathcal{H}_{1}$ or $\mathcal{H}_{2}$. Since $1_{H}$ is trivially invariant under the adjoint action we see that the vertex operator at the leftmost vertex is not affected by the additional face, either. By similar arguments one proves that $i_{V}$ intertwines the Hamiltonians, too.

For the sake of completeness we also give the maps $\left(i_{F}^{A, B}\right)^{\dagger}$ and $\left(i_{V}^{A}\right)^{\dagger}$ :

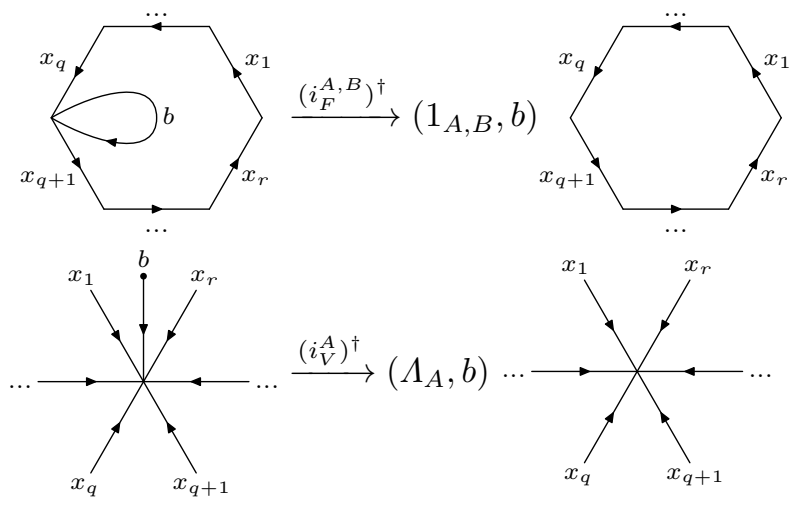

Now we define the unitary maps that allow for reconnecting edges of the underlying graph.

Proposition 4 (Unitaries). The linear maps $U_{F}$ and $U_{V}$ defined via

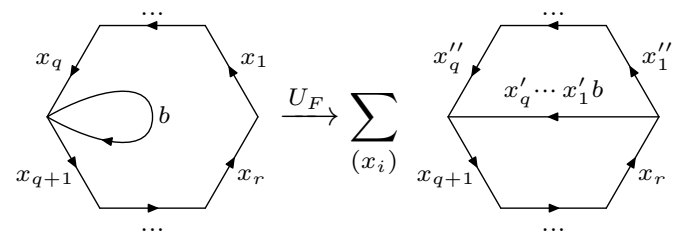


and

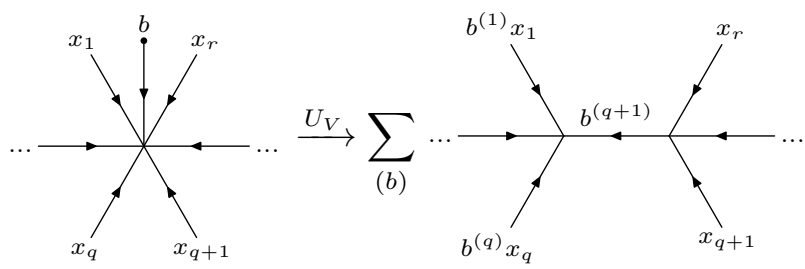

are unitary and their inverses read

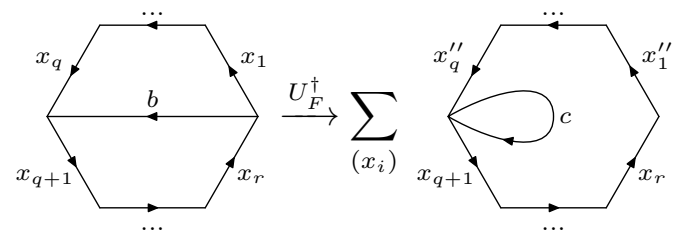

where $c=S\left(x_{q}^{\prime} \cdots x_{1}^{\prime}\right) b$ and

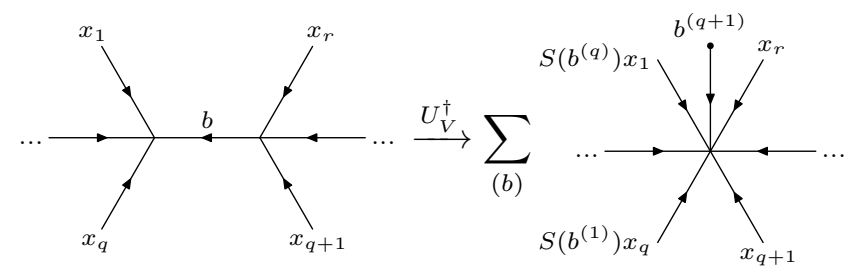

respectively.

Furthermore one has

$$
\begin{aligned}
U_{F} \mathcal{H}_{1} & =\mathcal{H}_{2} U_{F}, \\
U_{V} \mathcal{H}_{1} & =\mathcal{H}_{2} U_{V} .
\end{aligned}
$$

Proof. It is easy to check that $U_{F}^{\dagger}\left(U_{V}^{\dagger}\right)$ as defined above is both a left and a right inverse of the map $U_{F}\left(U_{V}\right)$. Indeed, leaving out the part $x_{q+1} \otimes \cdots \otimes x_{r}$ (which is unaffected by $U_{F}$ ) one has:

$$
\begin{aligned}
U_{F}^{\dagger} U_{F}\left(x_{1} \otimes \cdots \otimes x_{q} \otimes b\right) & =U_{F}^{\dagger}\left(\sum_{\left(x_{i}\right)} x_{1}^{\prime \prime} \otimes \cdots \otimes x_{q}^{\prime \prime} \otimes x_{q}^{\prime} \cdots x_{1}^{\prime} b\right) \\
& =\sum_{\left(x_{i}\right)} x_{1}^{\prime \prime \prime} \otimes \cdots \otimes x_{q}^{\prime \prime \prime} \otimes S\left(x_{q}^{\prime \prime} \cdots x_{1}^{\prime \prime}\right) x_{q}^{\prime} \cdots x_{1}^{\prime} b \\
& =\sum_{\left(x_{i}\right)} x_{1}^{\prime \prime \prime} \otimes \cdots \otimes x_{q}^{\prime \prime \prime} \otimes S\left(x_{1}^{\prime \prime}\right) \cdots S\left(x_{q}^{\prime \prime}\right) x_{q}^{\prime} \cdots x_{1}^{\prime} b \\
& =\sum_{\left(x_{i}\right)} x_{1}^{\prime \prime} \otimes \cdots \otimes x_{q}^{\prime \prime} \otimes \epsilon\left(x_{1}^{\prime}\right) \cdots \epsilon\left(x_{q}^{\prime}\right) b \\
& =x_{1} \otimes \cdots \otimes x_{q} \otimes b
\end{aligned}
$$


Similarly, one shows $U_{F} U_{F}^{\dagger}=$ id. Now

$$
\begin{aligned}
U_{V}^{\dagger} U_{V}\left(x_{1} \otimes \cdots \otimes x_{q} \otimes b\right) & =U_{V}^{\dagger} \sum_{(b)} b^{(1)} x_{1} \otimes \cdots \otimes b^{(q)} x_{q} \otimes b^{(q+1)} \\
& =\sum_{(b)} \sum_{(b(q+1)} S\left(\left(b^{(q+1)}\right)^{(q)}\right) b^{(1)} x_{1} \otimes \cdots \\
& \left.=\sum_{(b)} S\left(b^{(2 q)}\right) b^{(1)} x_{1} \otimes \cdots \otimes S\left(b^{(q+1)}\right)^{(1)}\right) b^{(q)} x_{q} \otimes\left(b^{(q+1)}\right) b^{(q)} x_{q} \otimes b^{(q+1)} \\
& =\sum_{(b)} S\left(b^{(2 q-1)}\right) b^{(1)} x_{1} \otimes \cdots \otimes S\left(b^{(q)}\right) b^{(q-1)} x_{q-1} \\
& =\sum_{(b)} S\left(b^{\prime \prime}\right) b^{\prime} x_{1} \otimes x_{2} \otimes \cdots \otimes x_{q} \otimes b^{\prime \prime \prime} \\
& =x_{1} \otimes \cdots \otimes x_{q} \otimes b .
\end{aligned}
$$

and by the same token one proves $U_{V} U_{V}^{\dagger}=$ id.

For the first claim it remains to show that for all $x_{i}, y_{i}, b, c \in H$ the inner product 1119 is invariant under $U_{F}\left(U_{V}\right)$ :

$$
\begin{aligned}
& \left(U_{F}\left(x_{1} \otimes \cdots \otimes x_{q} \otimes b\right), U_{F}\left(y_{1} \otimes \cdots \otimes y_{q} \otimes c\right)\right) \\
& =\left(\sum_{\left(x_{i}\right)} x_{1}^{\prime \prime} \otimes \cdots \otimes x_{q}^{\prime \prime} \otimes x_{q}^{\prime} \cdots x_{1}^{\prime} b, \sum_{\left(y_{i}\right)} y_{1}^{\prime \prime} \otimes \cdots \otimes y_{q}^{\prime \prime} \otimes y_{q}^{\prime} \cdots y_{1}^{\prime} c\right) \\
& =\sum_{\left(x_{i}\right)\left(y_{i}\right)}\left(x_{q}^{\prime} \cdots x_{1}^{\prime} b, y_{q}^{\prime} \cdots y_{1}^{\prime} c\right) \prod_{j=1}^{q}\left(x_{j}^{\prime \prime}, y_{j}^{\prime \prime}\right) \\
& =\sum_{\left(x_{i}\right)\left(y_{i}\right)} \phi\left(b^{*}\left(x_{1}^{*}\right)^{\prime} \cdots\left(x_{q}^{*}\right)^{\prime} y_{q}^{\prime} \cdots y_{1}^{\prime} c\right) \phi\left(\left(x_{q}^{*}\right)^{\prime \prime} y_{q}^{\prime \prime}\right) \prod_{j=1}^{q-1} \phi\left(\left(x_{j}^{*}\right)^{\prime \prime} y_{j}^{\prime \prime}\right) \\
& =\left(x_{q}, y_{q}\right) \sum_{\left(x_{i}\right)\left(y_{i}\right)} \phi\left(b^{*}\left(x_{1}^{*}\right)^{\prime} \cdots\left(x_{q-1}^{*}\right)^{\prime} y_{q-1}^{\prime} \cdots y_{1}^{\prime} c\right) \prod_{j=1}^{q-1} \phi\left(\left(x_{j}^{*}\right)^{\prime \prime} y_{j}^{\prime \prime}\right) \\
& =(b, c) \prod_{j=1}^{q}\left(x_{j}, y_{j}\right) \\
& =\left(x_{1} \otimes \cdots \otimes x_{q} \otimes b, y_{1} \otimes \cdots \otimes y_{q} \otimes c\right) .
\end{aligned}
$$

Note that we used property (38) of the Haar integral $\phi$ in the fifth line. Furthermore we have

$$
\begin{aligned}
& \left(U_{V}\left(x_{1} \otimes \cdots \otimes x_{q} \otimes b\right), U_{V}\left(y_{1} \otimes \cdots \otimes y_{q} \otimes c\right)\right) \\
& =\left(\sum_{(b)} b^{(1)} x_{1} \otimes \cdots \otimes b^{(q)} x_{q} \otimes b^{(q+1)}, \sum_{(c)} c^{(1)} y_{1} \otimes \cdots \otimes c^{(q)} y_{q} \otimes c^{(q+1)}\right)
\end{aligned}
$$




$$
\begin{aligned}
= & \sum_{(b)(c)}\left(b^{(q+1)}, c^{(q+1)}\right) \prod_{j=1}^{q}\left(b^{(j)} x_{j}, c^{(j)} y_{j}\right) \\
= & \sum_{(b)(c)} \phi\left(\left(b^{(q+1)}\right)^{*} c^{(q+1)}\right) \prod_{j=1}^{q} \phi\left(x_{j}^{*}\left(b^{(j)}\right)^{*} c^{(j)} y_{j}\right) \\
= & \sum_{\left(b^{*} c\right)} \phi\left(\left(b^{*} c\right)^{(q+1)}\right) \prod_{j=1}^{q} \phi\left(x_{j}^{*}\left(b^{*} c\right)^{(j)} y_{j}\right) \\
= & \left(x_{q}, y_{q}\right) \sum_{\left(b^{*} c\right)} \phi\left(\left(b^{*} c\right)^{(q)}\right) \prod_{j=1}^{q-1} \phi\left(x_{j}^{*}\left(b^{*} c\right)^{(j)} y_{j}\right) \\
= & (b, c) \prod_{j=1}^{q}\left(x_{j}, y_{j}\right) \\
= & \left(x_{1} \otimes \cdots \otimes x_{q} \otimes b, y_{1} \otimes \cdots \otimes y_{q} \otimes c\right) .
\end{aligned}
$$

For the second claim, one needs to show that $U_{F}\left(U_{V}\right)$ commutes appropriately with the terms $A(s)$ and $B(p)$ in the Hamiltonians $\mathcal{H}_{i}$. This can be done directly for those faces which are complete in 178 ). Stating this more precisely we have:

$$
U_{F} B\left(p_{i}\right)=B\left(p_{i}^{\prime}\right) U_{F} \quad i \in\{1,2\}
$$

where faces are labelled as follows:
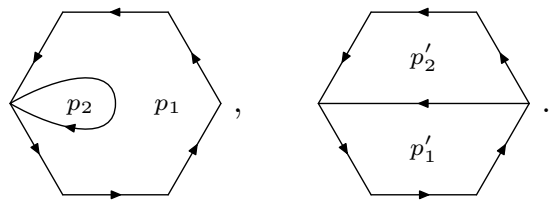

Indeed, consider

$$
\begin{aligned}
& U_{F} B\left(p_{2}\right)\left(x_{1} \otimes \cdots \otimes x_{q} \otimes b\right) \\
&= U_{F}\left(x_{1} \otimes \cdots \otimes x_{q} \otimes \sum_{(b)} \phi\left(b^{\prime}\right) b^{\prime \prime}\right) \\
&= \sum_{\left(x_{i}\right)(b)} x_{1}^{\prime \prime} \otimes \cdots \otimes x_{q}^{\prime \prime} \otimes x_{q}^{\prime} \cdots x_{1}^{\prime} b^{\prime} \phi\left(b^{\prime \prime}\right) \\
&= \sum_{\left(x_{i}\right)(b)} \phi\left(S\left(b^{\prime \prime}\right) S\left(x_{1}^{(2)}\right) \cdots S\left(x_{q}^{(2)}\right) x_{q}^{(3)} \cdots x_{1}^{(3)}\right) x_{1}^{(4)} \otimes \cdots \otimes x_{q}^{(4)} \otimes x_{q}^{(1)} \cdots x_{1}^{(1)} b^{\prime} \\
&= \sum_{\left(x_{i}\right)(b)} \phi\left(S\left(x_{q}^{(2)} \cdots x_{1}^{(2)} b^{\prime \prime}\right) x_{q}^{(3)} \cdots x_{1}^{(3)}\right) x_{1}^{(4)} \otimes \cdots \otimes x_{q}^{(4)} \otimes x_{q}^{(1)} \cdots x_{1}^{(1)} b^{\prime} \\
&=(\mathrm{id} \otimes \cdots \otimes \mathrm{id} \otimes S) \sum_{\left(x_{i}\right)(b)} \sum_{\left(S\left(x_{q}^{\prime} \cdots x_{1}^{\prime} b\right)\right)} \phi\left(S\left(x_{q}^{\prime} \cdots x_{1}^{\prime} b\right)^{\prime} x_{q}^{\prime \prime} \cdots x_{1}^{\prime \prime}\right) \\
& x_{1}^{\prime \prime \prime} \otimes \cdots \otimes x_{q}^{\prime \prime \prime} \otimes S\left(x_{q}^{\prime} \cdots x_{1}^{\prime} b\right)^{\prime \prime}
\end{aligned}
$$




$$
\begin{aligned}
& =B\left(p_{2}^{\prime}\right)\left(x_{1}^{\prime \prime} \otimes \cdots \otimes x_{q}^{\prime \prime} \otimes x_{q}^{\prime} \cdots x_{1}^{\prime} b\right) \\
& =B\left(p_{2}^{\prime}\right) U_{F}\left(x_{1} \otimes \cdots \otimes x_{q} \otimes b\right) .
\end{aligned}
$$

Note that in the fourth line we repeatedly used the definition of the antipode. Hence both $B\left(p_{2}\right)$ and $B\left(p_{2}^{\prime}\right)$ project $b$ onto the value $\phi(b) 1_{H}$. In the following we may therefore assume that $b$ is replaced by $\phi(b) 1_{H}$. We now analyze the other complete face:

$$
\begin{aligned}
& U_{F} B\left(p_{1}\right)\left(x_{1} \otimes \cdots \otimes x_{q} \otimes \phi(b) 1_{H} \otimes x_{q+1} \otimes \cdots \otimes x_{r}\right) \\
& =\phi(b) U_{F}\left(\sum_{\left(x_{i}\right)} \phi\left(x_{r}^{\prime} \cdots x_{1}^{\prime}\right) x_{1}^{\prime \prime} \otimes \cdots \otimes x_{q}^{\prime \prime} \otimes 1_{H} \otimes x_{q+1}^{\prime \prime} \otimes \cdots \otimes x_{r}^{\prime \prime}\right) \\
& =\phi(b) \sum_{\left(x_{i}\right)} \phi\left(x_{r}^{\prime} \cdots x_{1}^{\prime}\right) x_{1}^{\prime \prime \prime} \otimes \cdots \otimes x_{q}^{\prime \prime \prime} \otimes x_{q}^{\prime \prime} \cdots x_{1}^{\prime \prime} \otimes x_{q+1}^{\prime \prime} \otimes \cdots \otimes x_{r}^{\prime \prime} \\
& =\phi(b) \sum_{\left(x_{i}\right)} \sum_{\left(x_{q}^{\prime} \cdots x_{1}^{\prime}\right)} \phi\left(x_{r}^{\prime} \cdots x_{q+1}^{\prime}\left(x_{q}^{\prime} \cdots x_{1}^{\prime}\right)^{\prime}\right) x_{1}^{\prime \prime} \otimes \cdots \otimes x_{q}^{\prime \prime} \otimes\left(x_{q}^{\prime} \cdots x_{1}^{\prime}\right)^{\prime \prime} \\
& \otimes x_{q+1}^{\prime \prime} \otimes \cdots \otimes x_{r}^{\prime \prime} \\
& =\phi(b) B\left(p_{1}^{\prime}\right)\left(\sum_{\left(x_{i}\right)} x_{1}^{\prime \prime} \otimes \cdots \otimes x_{q}^{\prime \prime} \otimes x_{q}^{\prime} \cdots x_{1}^{\prime} \otimes x_{q+1} \otimes \cdots \otimes x_{r}\right) \\
& =B\left(p_{1}\right) U_{F}\left(x_{1} \otimes \cdots \otimes x_{q} \otimes \phi(b) 1_{H} \otimes x_{q+1} \otimes \cdots \otimes x_{r}\right) .
\end{aligned}
$$

For those surrounding faces which are affected by $U_{F}$ one may easily prove

$$
\left[U_{F},\left(T_{+}^{f}\right)_{i}\right]=0 \quad i \in\{1, \ldots, q\}
$$

for any $f \in X$. Here $\left(T_{+}^{f}\right)_{i}$ denotes the action on the i-th tensor factor.

Those incomplete vertices in (178) which are affected by $U_{F}$ can be dealt with if the following is true for all $a \in H, i \in\{1, \ldots, q-1\}$ and $b \propto 1_{H}$ :

$$
\begin{aligned}
U_{F}\left(L_{-}^{a}\right)_{1} & =\left(\sum_{(a)}\left(L_{-}^{a^{\prime}}\right)_{1} \otimes\left(L_{-}^{a^{\prime \prime}}\right)_{\diamond}\right) U_{F} \\
{\left[U_{F}, \sum_{(a)}\left(L_{+}^{a^{\prime}}\right)_{i} \otimes\left(L_{-}^{a^{\prime \prime}}\right)_{i+1}\right] } & =0 \\
U_{F}\left(\sum_{(a)}\left(L_{+}^{a^{\prime \prime}}\right)_{q} \otimes \operatorname{ad}\left(a^{\prime}\right)_{\diamond}\right) & =\left(\sum_{(a)}\left(L_{+}^{a^{\prime \prime}}\right)_{q} \otimes\left(L_{+}^{a^{\prime}}\right)_{\diamond}\right) U_{F} .
\end{aligned}
$$

Here $\diamond$ denotes the bubble edge that is reconnected by $U_{F}$. While it is easy to show the first two equations, the remaining one needs some more attention:

$$
\begin{aligned}
& U_{F}\left(\sum_{(a)}\left(L_{+}^{a^{\prime \prime}}\right)_{q} \otimes \operatorname{ad}\left(a^{\prime}\right)_{\diamond}\right)\left(x_{1} \otimes \cdots \otimes x_{q} \otimes \phi(b) 1_{H}\right) \\
& =U_{F}\left(\sum_{(a)} x_{1} \otimes \cdots \otimes x_{q-1} \otimes a^{\prime \prime} x_{q} \otimes \phi(b) \operatorname{ad}\left(a^{\prime}\right)\left(1_{H}\right)\right) \\
& =\phi(b) U_{F}\left(x_{1} \otimes \cdots \otimes x_{q-1} \otimes a x_{q} \otimes 1_{H}\right)
\end{aligned}
$$




$$
\begin{aligned}
& =\phi(b) \sum_{\left(x_{i}\right)(a)} x_{1}^{\prime \prime} \otimes \cdots \otimes x_{q-1}^{\prime \prime} \otimes a^{\prime \prime} x_{q}^{\prime \prime} \otimes a^{\prime} x_{q}^{\prime} \ldots x_{1}^{\prime} \\
& =\left(\sum_{(a)}\left(L_{+}^{a^{\prime \prime}}\right)_{q} \otimes\left(L_{+}^{a^{\prime}}\right)_{\diamond}\right) U_{F}\left(x_{1} \otimes \cdots \otimes x_{q} \otimes \phi(b) 1_{H}\right)
\end{aligned}
$$

This completes the proof of 182 .

The proof of $(183)$ is analogous and left as an exercise for the reader.

Corollary 1 (Isometries). The linear maps $I_{F}^{A, B}:=U_{F} \circ i_{F}^{A, B}$,

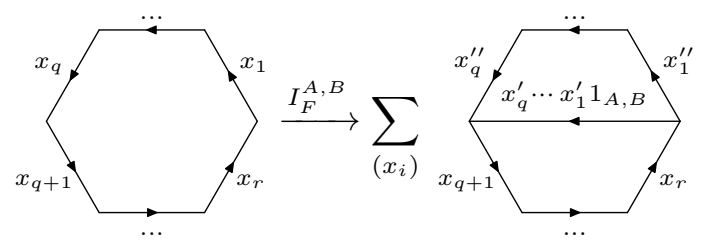

and $I_{V}^{A}:=U_{V} \circ i_{V}^{A}$,

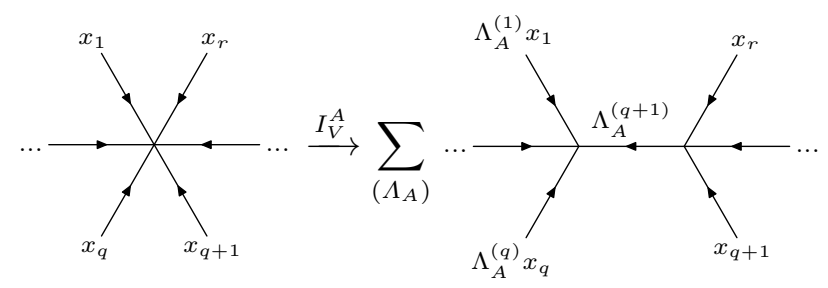

are isometries. Furthermore, for $I_{F}:=I_{F}^{H, H^{*}}$ and $I_{V}:=I_{V}^{H}$ one has

$$
\begin{aligned}
I_{F} \mathcal{H}_{1} & =\mathcal{H}_{2} I_{F}, \\
I_{V} \mathcal{H}_{1} & =\mathcal{H}_{2} I_{V} .
\end{aligned}
$$

Put differently, the collection of these maps allows to move between the $\mathrm{D}(H)$ models defined on two arbitrary graphs which are embedded in the same surface. Furthermore, all maps are local, i.e. they cannot change nonlocal topological quantum numbers.

5.2. Transforming hierarchy states. In the preceding section we have learned that the set $\left\{I_{F}, I_{V}\right\}$ of isometries maps the ground state subspaces of generalized quantum double models on related graphs onto each other. We would like to prove that this not only holds for the entire subspace but also for individual ground states. Namely, the isometries $\left\{I_{F}, I_{V}\right\}$ precisely identify the Hopf tensor network states $\left|\psi_{\Gamma_{i}}\right\rangle$ as given by Theorem 3 for the different graphs $\Gamma_{i}$.

Keeping our focus on states we will furthermore show (for a special case) that the hierarchy of isometries $\left\{I_{F}^{A, B}, I_{V}^{A}\right\}$ has the very same effect on the hierarchy of states $\left|\psi_{\Gamma_{i}}^{A, B}\right\rangle$ as given by Definition 5 . In other words, identifying these states in an isometric fashion is as simple as adding or removing edges from the underlying graph and adjusting the tensor trace canonically. 
Once we act with the isometries on our hierarchy of Hopf tensor network states it will become clear that the quantum states defined in Section 4.2 are not properly normalized. We can (partially) remedy this situation by choosing integrals of unit norm instead of the Haar integrals $h_{A}$ and $\phi_{B}$. In other words, we need to include a factor of $\left\|h_{A}\right\|^{-1}$ for each edge and a factor of $\left\|\phi_{B}\right\|^{-1}$ for each (complete) face of a Hopf tensor network state. It is understood that from now on all Hopf tensor network states will be normalized in this fashion without change of notation unless otherwise stated.

We now fix some notation in the following definition so that we can easily refer to pieces of Hopf tensor network states later on.

Definition 6. Let $A \subset H, B \subset X$ Hopf subalgebras, $h_{A} \in A, \phi_{B} \in B$ their respective Haar integrals and let $f_{1}, \ldots, f_{r} \in B$. Set

$$
\left|\psi_{p}^{A, B}\left(\left\{f_{i}\right\}\right)\right\rangle:=\frac{1}{\left\|h_{A}\right\|^{r}\left\|\phi_{B}\right\|}
$$




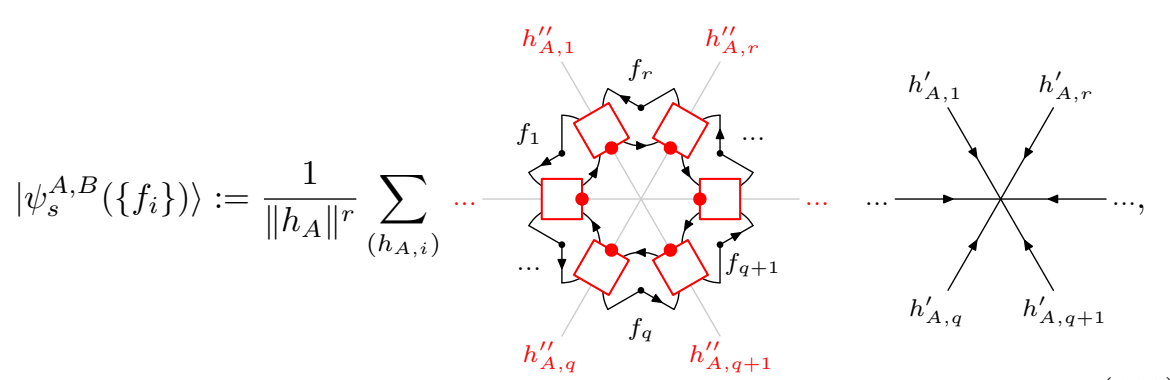

and

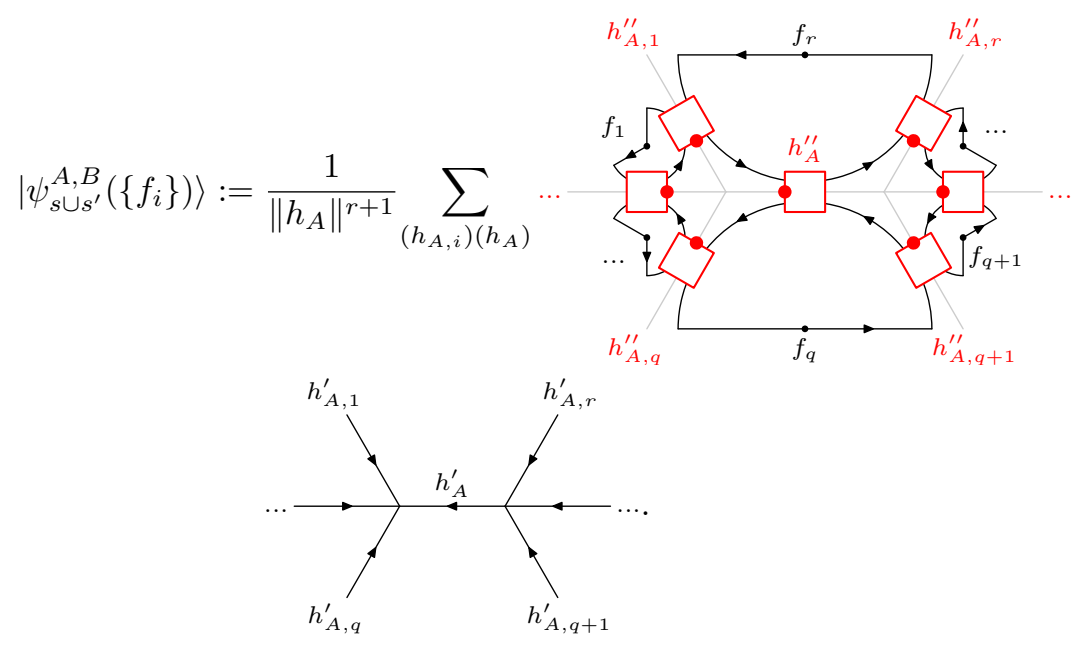

As before we simply write $\left|\psi_{\Gamma}\left(f_{1}, \ldots, f_{r}\right)\right\rangle$ whenever $A=H$ and $B=X$. Note that in this case one deals with a fragment of a quantum double model ground state!

\section{Proposition 5.}

$$
\begin{aligned}
I_{F}\left|\psi_{p}\left(f_{1}, \ldots, f_{r}\right)\right\rangle & =\left|\psi_{p \cup p^{\prime}}\left(f_{1}, \ldots, f_{r}\right)\right\rangle, \\
I_{V}^{A}\left|\psi_{s}^{A, B}\left(f_{1}, \ldots, f_{r}\right)\right\rangle & =\left|\psi_{s \cup s^{\prime}}^{A, B}\left(f_{1}, \ldots, f_{r}\right)\right\rangle .
\end{aligned}
$$

Proof. First let us prove the relation involving the face isometry. It is enough to consider a face which is bounded by two edges. In this case we first transform the right hand side of the equation in order to eliminate one of the virtual loops:

$$
\begin{aligned}
\left|\psi_{p \cup p^{\prime}}\left(f_{1}, f_{2}\right)\right\rangle & =|H|^{\frac{5}{2}} \sum_{\left(h_{i}\right)(h)} \phi\left(h_{1}^{\prime \prime \prime} h^{\prime \prime \prime}\right) \phi\left(h_{2}^{\prime \prime \prime} S\left(h^{\prime \prime}\right)\right) \prod_{j=1}^{2} f_{j}\left(S\left(h_{j}^{\prime \prime}\right)\right)\left|h_{1}^{\prime}\right\rangle \otimes\left|h^{\prime}\right\rangle \otimes\left|h_{2}^{\prime}\right\rangle \\
& =|H|^{\frac{3}{2}} \sum_{\left(h_{i}\right)} \phi\left(h_{2}^{\prime \prime \prime} h_{1}^{(3)}\right) f_{1}\left(S\left(h_{1}^{(2)}\right)\right) f_{2}\left(S\left(h_{2}^{\prime \prime}\right)\right)\left|h_{1}^{(1)}\right\rangle \otimes\left|S\left(h_{1}^{(4)}\right)\right\rangle \otimes\left|h_{2}^{\prime}\right\rangle \\
& =|H|^{\frac{3}{2}} \sum_{\left(h_{i}\right)} \phi\left(h_{2}^{\prime \prime \prime} h_{1}^{(4)}\right) f_{1}\left(S\left(h_{1}^{(3)}\right)\right) f_{2}\left(S\left(h_{2}^{\prime \prime}\right)\right)\left|h_{1}^{(2)}\right\rangle \otimes\left|S\left(h_{1}^{(1)}\right)\right\rangle \otimes\left|h_{2}^{\prime}\right\rangle
\end{aligned}
$$


where in the second line we employed Lemma 5

In diagrammatic notation the above amounts to

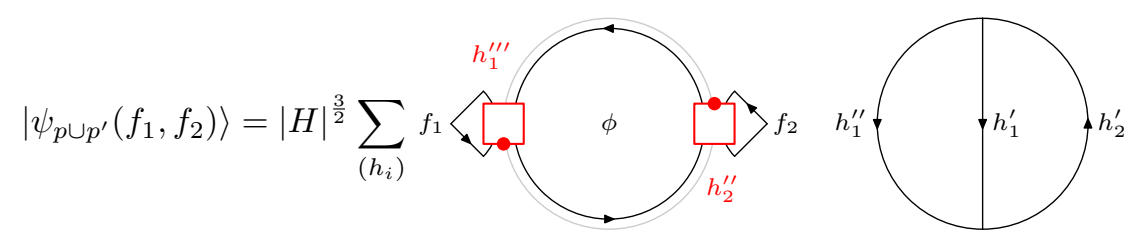

which proves the claim about the face isometry.

As for the vertex isometries we can in fact assume $A=H$ without loss of generality. By the same token we will never make use of $f_{i} \in B$ in the following argument, hence it suffices to assume $B=X$, too. We begin with the state on the original graph:

$$
\left|\psi_{s}\left(f_{1}, \ldots, f_{r}\right)\right\rangle=\|h\|^{-r} \sum_{\left(h_{i}\right)} \prod_{j=1}^{r} f_{j}\left(S\left(h_{j}^{\prime \prime}\right) h_{j+1}^{\prime \prime \prime}\right)\left|h_{1}^{\prime}\right\rangle \otimes \cdots \otimes\left|h_{r}^{\prime}\right\rangle .
$$

Applying $I_{V}$ yields:

$$
\begin{aligned}
& \|h\|^{r+1} I_{V}\left|\psi_{s}\left(f_{1}, \ldots, f_{r}\right)\right\rangle \\
& =\sum_{(h)\left(h_{i}\right)} \prod_{j=1}^{r} f_{j}\left(S\left(h_{j}^{\prime \prime}\right) h_{j+1}^{\prime \prime \prime}\right) \bigotimes_{k=1}^{q}\left|h^{(k)} h_{k}^{\prime}\right\rangle \otimes\left|h^{(q+1)}\right\rangle \otimes\left|h_{q+1}^{\prime}\right\rangle \otimes \cdots \otimes\left|h_{r}^{\prime}\right\rangle \\
& =\sum_{\left(h_{i}\right)(h)} f_{r}\left(S\left(h_{r}^{\prime \prime}\right) h_{1}^{\prime \prime \prime}\right) \prod_{j=1}^{q} f_{j}\left(S\left(h_{j}^{\prime \prime}\right) h_{j+1}^{\prime \prime \prime}\right) \bigotimes_{k=1}^{q}\left|h^{(k)} h_{k}^{\prime}\right\rangle \otimes\left|h^{(q+1)}\right\rangle \otimes \cdots
\end{aligned}
$$

where in the last line we have abbreviated the remaining tensor factor

$$
\prod_{j=q+1}^{r-1} f_{j}\left(S\left(h_{j}^{\prime \prime}\right) h_{j+1}^{\prime \prime \prime}\right) \bigotimes_{k=q+1}^{r}\left|h_{k}^{\prime}\right\rangle
$$

by the trailing ellipsis. In order to simplify notation we will continue to do so in the following. Hence:

$$
\begin{aligned}
& \|h\|^{r+1} I_{V}\left|\psi_{s}\left(f_{1}, \ldots, f_{r}\right)\right\rangle \\
& =\sum_{\left(h_{i}\right)(h)} f_{r}\left(S\left(h_{r}^{\prime \prime}\right) h_{1}^{\prime \prime \prime}\right) f_{1}\left(S\left(h_{1}^{\prime \prime}\right) h_{2}^{\prime \prime \prime}\right) \prod_{j=2}^{q} f_{j}\left(S\left(h_{j}^{\prime \prime}\right) h_{j+1}^{\prime \prime \prime}\right) \\
& \quad\left|h^{(1)} h_{1}^{\prime}\right\rangle \otimes \cdots \otimes\left|h^{(q)} h_{q}^{\prime}\right\rangle \otimes\left|h^{(q+1)}\right\rangle \otimes \cdots \\
& =\sum_{\left(h_{i}\right)(h)} f_{r}\left(S\left(h_{r}^{\prime \prime}\right) S\left(h^{(1)}\right) h_{1}^{\prime \prime \prime}\right) f_{1}\left(S\left(h_{1}^{\prime \prime}\right) h^{(2)} h_{2}^{\prime \prime \prime}\right) f_{2}\left(S\left(h_{2}^{\prime \prime}\right) h_{3}^{\prime \prime \prime}\right) \prod_{j=3}^{q} f_{j}\left(S\left(h_{j}^{\prime \prime}\right) h_{j+1}^{\prime \prime \prime}\right) \\
& \quad\left|h_{1}^{\prime}\right\rangle \otimes\left|h^{(3)} h_{2}^{\prime}\right\rangle \otimes \cdots \otimes\left|h^{(q+1)} h_{q}^{\prime}\right\rangle \otimes\left|h^{(q+2)}\right\rangle \otimes \cdots
\end{aligned}
$$




$$
\begin{aligned}
= & \sum_{\left(h_{i}\right)(h)} f_{r}\left(S\left(h_{r}^{\prime \prime}\right) S\left(h^{(1)}\right) h_{1}^{\prime \prime \prime}\right) f_{1}\left(S\left(h_{1}^{\prime \prime}\right) h_{2}^{\prime \prime \prime}\right) f_{2}\left(S\left(h_{2}^{\prime \prime}\right) h^{(2)} h_{3}^{\prime \prime \prime}\right) \prod_{j=3}^{q} f_{j}\left(S\left(h_{j}^{\prime \prime}\right) h_{j+1}^{\prime \prime \prime}\right) \\
& \left|h_{1}^{\prime}\right\rangle \otimes\left|h_{2}^{\prime}\right\rangle \otimes\left|h^{(3)} h_{3}^{\prime}\right\rangle \otimes \cdots \otimes\left|h^{(q)} h_{q}^{\prime}\right\rangle \otimes\left|h^{(q+1)}\right\rangle \otimes \cdots \\
= & \sum_{\left(h_{i}\right)(h)} f_{r}\left(S\left(h_{r}^{\prime \prime}\right) S\left(h^{(1)}\right) h_{1}^{\prime \prime \prime}\right) f_{q}\left(S\left(h_{q}^{\prime \prime}\right) h^{(2)} h_{q+1}^{\prime \prime \prime}\right) \prod_{j=1}^{q-1} f_{j}\left(S\left(h_{j}^{\prime \prime}\right) h_{j+1}^{\prime \prime \prime}\right) \\
& \left|h_{1}^{\prime}\right\rangle \otimes \cdots \otimes\left|h_{q}^{\prime}\right\rangle \otimes\left|h^{(3)}\right\rangle \otimes \cdots \\
= & \sum_{\left(h_{i}\right)(h)} f_{q}\left(S\left(h_{q}^{\prime \prime}\right) h^{\prime \prime \prime} h_{q+1}^{\prime \prime \prime}\right) f_{r}\left(S\left(h_{r}^{\prime \prime}\right) S\left(h^{\prime \prime}\right) h_{1}^{\prime \prime \prime}\right) \prod_{j \neq q, r} f_{j}\left(S\left(h_{j}^{\prime \prime}\right) h_{j+1}^{\prime \prime \prime}\right) \\
& \left|h_{1}^{\prime}\right\rangle \otimes \cdots \otimes\left|h_{q}^{\prime}\right\rangle \otimes\left|h^{\prime}\right\rangle \otimes\left|h_{q+1}^{\prime}\right\rangle \otimes \cdots \otimes\left|h_{r}^{\prime}\right\rangle \\
= & \|h\|^{r+1}\left|\psi_{s \cup s^{\prime}}\left(f_{1}, \ldots, f_{r}\right)\right\rangle .
\end{aligned}
$$

Note that from the third line on we repeatedly applied Lemma 4.

Unfortunately, we do not know how arbitrary hierarchy states $\left|\psi_{\Gamma}^{A, B}\right\rangle$ are affected by the associated face isometries $I_{F}^{A, B}$ for a general finite-dimensional Hopf $C^{*}$-algebra. However, for $H=\mathbb{C} G$ we can state the following and leave the proof to the reader.

Lemma 3. Let $K \subset G$ a subgroup and $N \triangleleft G$ a normal subgroup. Furthermore let $f_{i} \in \mathbb{C}^{G / N}$. Then

$$
I_{F}^{K, N}\left|\psi_{p}^{K, N}\left(f_{1}, \ldots, f_{r}\right)\right\rangle=\sqrt{\frac{|K N|}{|G|}}\left|\psi_{p \cup p^{\prime}}^{K, N}\left(f_{1}, \ldots, f_{r}\right)\right\rangle .
$$

The above lemma shows that unless one draws the quantum state from the top of the hierarchy one needs to include an additional factor of $\sqrt{|K N| /|G|}$ for each face to insure proper relative normalization of the Hopf tensor network states. In order to fix the normalization absolutely we may calculate the norm of a single (preferably small) Hopf tensor network state on a given surface. Since all other states on the same surface can be reached from this initial one via isometries their norm will be determined automatically. It turns out that the absolute normalization factor is entirely a property of the surface the Hopf tensor network state is embedded in. For example, absorbing the additional factor per face into the definition of the Hopf tensor network states one has for arbitrary graphs $\Gamma$ on $S^{2}$

$$
\|\left|\psi_{\Gamma}^{K, N}\right\rangle \|=\sqrt{|K N / N|}
$$

while on $T^{2}$ one has

$$
\|\left|\psi_{\Gamma}^{K, N}\right\rangle \|=\frac{1}{\sqrt{|K N / N|}} \sqrt{\sum_{g \in K N / N}\left|C_{K N / N}(g)\right|} .
$$

Here $C_{G}(g)$ denotes the centralizer of the element $g$ in the group $G$. In order to keep the following discussion as general as possible we will stick to relative normalization unless otherwise noted. 
5.3. Entanglement entropy. Having defined the isometries $I_{F}$ and $I_{V}$ we finally embark on an exact calculation of the entanglement entropy for a simply connected region $R$ on $S^{2}$. We focus on the Hopf tensor network state $\left|\psi_{\Gamma}\right\rangle$.

In the following we show how the inner product between two face pieces of the Hopf tensor network state $\left|\psi_{\Gamma}\right\rangle$ depends on the boundary degrees of freedom.

Proposition 6. Let $f_{i}, g_{i} \in X$ and $r=|\partial R|$. Then

$$
\left\langle\psi_{p}\left(f_{1}, \ldots, f_{r}\right) \mid \psi_{p}\left(g_{1}, \ldots, g_{r}\right)\right\rangle=|H|^{-r} \sum_{\left(h_{i}\right)} \phi\left(h_{1}^{\prime} \cdots h_{r}^{\prime}\right) \prod_{j=1}^{r}\left(g_{j} f_{j}^{*}\right)\left(h_{j}^{\prime \prime}\right) .
$$

Proof. Let $\lambda_{i}=h_{i}=h$ the Haar integral of $H$. Then from 155 we get

$$
\begin{aligned}
\left\langle\psi_{p}\left(f_{1}, \ldots, f_{r}\right) \mid \psi_{p}\left(g_{1}, \ldots, g_{r}\right)\right\rangle= & \sum_{\left(h_{i}\right)\left(\lambda_{i}\right)} \overline{\phi\left(h_{r}^{\prime \prime \prime} \cdots h_{1}^{\prime \prime \prime}\right)} \phi\left(\lambda_{r}^{\prime \prime \prime} \cdots \lambda_{1}^{\prime \prime \prime}\right) \\
& \prod_{j=1}^{r} \overline{f_{j}\left(S\left(h_{j}^{\prime \prime}\right)\right)} g_{j}\left(S\left(\lambda_{j}^{\prime \prime}\right)\right)\left\langle h_{j}^{\prime} \mid \lambda_{j}^{\prime}\right\rangle \\
= & \sum_{\left(h_{i}\right)\left(\lambda_{i}\right)} \phi\left(h_{1}^{\prime \prime \prime} \cdots h_{r}^{\prime \prime \prime}\right) \phi\left(\lambda_{r}^{\prime \prime \prime} \cdots \lambda_{1}^{\prime \prime \prime}\right) \\
& \prod_{j=1}^{r} f_{j}^{*}\left(h_{j}^{\prime \prime}\right) g_{j}\left(S\left(\lambda_{j}^{\prime \prime}\right)\right) \phi\left(h_{j}^{\prime} \lambda_{j}^{\prime}\right)
\end{aligned}
$$

where we used (119), the properties of $h$ and the involution $*$ as well as (51) for the second line. Employing Lemma 6 we can simplify this to

$$
\begin{aligned}
\left\langle\psi_{p}\left(f_{1}, \ldots, f_{r}\right) \mid \psi_{p}\left(g_{1}, \ldots, g_{r}\right)\right\rangle= & |H|^{-1} \sum_{\left(h_{i}\right)\left(\lambda_{i}\right)} \phi\left(h_{1}^{\prime \prime \prime} \cdots h_{r}^{\prime \prime \prime}\right) \phi\left(\lambda_{r}^{\prime \prime \prime} \cdots \lambda_{2}^{\prime \prime \prime} S\left(h_{1}^{\prime}\right)\right) \\
& \left(g_{1} f_{1}^{*}\right)\left(h_{1}^{\prime \prime}\right) \prod_{j=2}^{r} f_{j}^{*}\left(h_{j}^{\prime \prime}\right) g_{j}\left(S\left(\lambda_{j}^{\prime \prime}\right)\right) \phi\left(h_{j}^{\prime} \lambda_{j}^{\prime}\right) \\
= & |H|^{-r} \sum_{\left(h_{i}\right)} \phi\left(h_{1}^{\prime \prime \prime} \cdots h_{r}^{\prime \prime \prime}\right) \phi\left(S\left(h_{1}^{\prime} \cdots h_{r}^{\prime}\right)\right) \\
& \prod_{j=1}^{r}\left(g_{j} f_{j}^{*}\right)\left(h_{j}^{\prime \prime}\right) .
\end{aligned}
$$

Finally, the claim follows from $\phi^{2}=\phi$.

Analogously, we are interested in the inner product between vertex pieces of the same Hopf tensor network state.

Proposition 7. Let $f_{i}, g_{i} \in H^{*}$ and $r=|\partial R|$. Then

$$
\left\langle\psi_{s}\left(f_{1}, \ldots, f_{r}\right) \mid \psi_{s}\left(g_{1}, \ldots, g_{r}\right)\right\rangle=|H|^{-r} \sum_{\left(h_{i}\right)} \prod_{j=1}^{r} f_{j}^{*}\left(h_{j}^{(3)} S\left(h_{j+1}^{(4)}\right)\right) g_{j}\left(h_{j}^{(2)} S\left(h_{j+1}^{(1)}\right)\right) .
$$



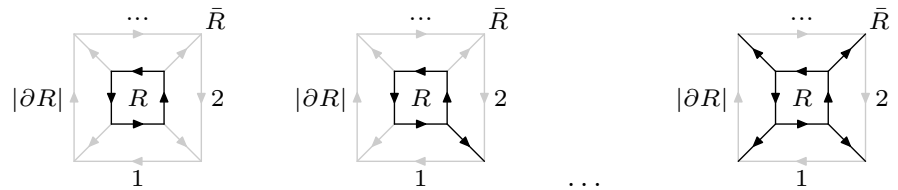

Fig. 7. Boundary graphs for a simply connected region $R \subset S^{2}$. Here black edges constitute the corresponding subgraph $\Gamma_{R}$ while grey edges belong to $\Gamma_{\bar{R}}$. These graphs can be reduced further (without touching the boundary) to minimal graphs.

Proof. Let again $\lambda_{i}=h_{i}=h$ the Haar integral of $H$. From 157 one has

$$
\begin{aligned}
\left\langle\psi_{s}\left(f_{1}, \ldots, f_{r}\right) \mid \psi_{s}\left(g_{1}, \ldots, g_{r}\right)\right\rangle & =\sum_{\left(h_{i}\right)} \sum_{\left(\lambda_{i}\right)} \prod_{j=1}^{r} \overline{f_{j}\left(S\left(h_{j}^{\prime \prime}\right) h_{j+1}^{\prime \prime \prime}\right)} g_{j}\left(S\left(\lambda_{j}^{\prime \prime}\right) \lambda_{j+1}^{\prime \prime \prime}\right)\left\langle h_{j}^{\prime} \mid \lambda_{j}^{\prime}\right\rangle \\
& =\sum_{\left(h_{i}\right)} \sum_{\left(\lambda_{i}\right)} \prod_{j=1}^{r} f_{j}^{*}\left(h_{j}^{\prime \prime} S\left(h_{j+1}^{\prime \prime \prime}\right)\right) g_{j}\left(S\left(\lambda_{j}^{\prime \prime}\right) \lambda_{j+1}^{\prime \prime \prime}\right) \phi\left(h_{j}^{\prime} \lambda_{j}^{\prime}\right) .
\end{aligned}
$$

Additionally, Lemma 6 yields

$$
\sum_{\left(\lambda_{i}\right)} \prod_{j=1}^{r} \phi\left(a_{j} \lambda_{j}^{\prime}\right) g_{j}\left(S\left(\lambda_{j}^{\prime \prime}\right) \lambda_{j+1}^{\prime \prime \prime}\right)=|H|^{-r} \sum_{\left(a_{i}\right)} \prod_{j=1}^{r} g_{j}\left(a_{j}^{\prime \prime} S\left(a_{j+1}^{\prime}\right)\right)
$$

where $a_{j} \in H$ is arbitrary. Armed with this identity it is easy to verify the claim.

We now state the main result of this section.

Theorem 4. Let $R \subset M$ a simply connected region and let $\Gamma_{R} \subset \Gamma$ the corresponding subgraph. Then for any $\alpha \geq 0$ the state $\left|\psi_{\Gamma}\right\rangle$ has the Rényi entanglement entropies

$$
S_{\alpha}\left(\rho_{R}\right)=|\partial R| \log |H|-\log |H| .
$$

In particular, the topological entanglement entropy reads

$$
\gamma=\log |H| \text {. }
$$

Proof. Without loss of generality we restrict to $M \simeq S^{2}$. Acting with the isometries $I_{F}$ and $I_{V}$ on $\left|\psi_{\Gamma}\right\rangle$ we may wipe out the bulk of both $R$ and $\bar{R}$ completely and reduce $\Gamma$ to one of the graphs shown in Figure 7 where we indicated several types of boundaries.

We further assume the first type of boundary in the following. In this case we can continue the isometric reduction to the graph $\Gamma_{0}$ given by

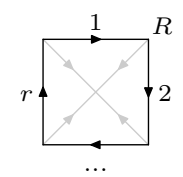

and for the resulting Hopf tensor network state on this graph Proposition 2 yields the natural splitting

$$
\left|\psi_{\Gamma_{0}}\right\rangle=\sum_{\left(\phi_{i}\right)}\left|\psi_{R}\left(\phi_{1}^{\prime}, \ldots, \phi_{r}^{\prime}\right)\right\rangle \otimes\left|\psi_{\bar{R}}\left(\phi_{1}^{\prime \prime}, \ldots, \phi_{r}^{\prime \prime}\right)\right\rangle
$$


where the states

$$
\begin{aligned}
\left|\psi_{R}\left(f_{1}, \ldots, f_{r}\right)\right\rangle & =\|h\|^{-r}\|\phi\|^{-1}\left|\psi_{p}\left(f_{1}, \ldots, f_{r}\right)\right\rangle \\
\left|\psi_{\bar{R}}\left(f_{1}, \ldots, f_{r}\right)\right\rangle & =\|h\|^{-r}\left|\psi_{s}\left(f_{r}, \ldots, f_{1}\right)\right\rangle
\end{aligned}
$$

are identical to 154 and 156 respectively up to normalization. Note that $r=|\partial R|$ denotes the length of the boundary.

We determine the reduced density operator $\rho_{R}=N^{-1} \widetilde{\rho}_{R}$ of the region $R$ as follows. Let $\phi_{i}=\varphi_{i}=\phi$ the Haar integral of $X$ and $h_{i}=\lambda_{i}=\kappa_{i}=h$ the Haar integral of $H$. Ignoring the normalization factor $N$ for a moment one has

$$
\begin{aligned}
& \widetilde{\rho}_{R}=\operatorname{tr}_{\bar{R}}\left(\left|\psi_{\Gamma_{0}}\right\rangle\left\langle\psi_{\Gamma_{0}}\right|\right) \\
& =\sum_{\left(\phi_{i}\right)\left(\varphi_{i}\right)}\left|\psi_{R}\left(\phi_{1}^{\prime}, \ldots, \phi_{r}^{\prime}\right)\right\rangle\left\langle\psi_{R}\left(\varphi_{1}^{\prime}, \ldots, \varphi_{r}^{\prime}\right)\right| \cdot\left\langle\psi_{\bar{R}}\left(\varphi_{1}^{\prime \prime}, \ldots, \varphi_{r}^{\prime \prime}\right) \mid \psi_{\bar{R}}\left(\phi_{1}^{\prime \prime}, \ldots, \phi_{r}^{\prime \prime}\right)\right\rangle \\
& =|H|^{r+1} \sum_{\left(\phi_{i}\right)\left(\varphi_{i}\right)}\left|\psi_{p}\left(\phi_{1}^{\prime}, \ldots, \phi_{r}^{\prime}\right)\right\rangle\left\langle\psi_{p}\left(\varphi_{1}^{\prime}, \ldots, \varphi_{r}^{\prime}\right)\right| \\
& \sum_{\left(h_{i}\right)} \prod_{j=1}^{r}\left(\varphi_{r+1-j}^{\prime \prime}\right)^{*}\left(h_{j}^{(3)} S\left(h_{j+1}^{(4)}\right)\right) \phi_{r+1-j}^{\prime \prime}\left(h_{j}^{(2)} S\left(h_{j+1}^{(1)}\right)\right) \\
& =|H|^{r+1} \sum_{\left(\phi_{i}\right)\left(\varphi_{i}\right)}\left(\sum_{\left(\lambda_{i}\right)} \phi\left(\lambda_{r}^{\prime \prime \prime} \cdots \lambda_{1}^{\prime \prime \prime}\right) \prod_{k=1}^{r} \phi_{k}^{\prime}\left(S\left(\lambda_{k}^{\prime \prime}\right)\right)\left|\lambda_{1}^{\prime}, \ldots, \lambda_{r}^{\prime}\right\rangle\right) \\
& \left(\sum_{\left(\kappa_{i}\right)} \overline{\phi\left(\kappa_{r}^{\prime \prime \prime} \cdots \kappa_{1}^{\prime \prime \prime}\right)} \prod_{l=1}^{r} \overline{\varphi_{l}^{\prime}\left(S\left(\kappa_{l}^{\prime \prime}\right)\right)}\left\langle\kappa_{1}^{\prime}, \ldots, \kappa_{r}^{\prime}\right|\right) \\
& \sum_{\left(h_{i}\right)} \prod_{j=1}^{r}\left(\varphi_{j}^{\prime \prime}\right)^{*}\left(h_{j}^{(3)} S\left(h_{j-1}^{(4)}\right)\right) \phi_{j}^{\prime \prime}\left(h_{j}^{(2)} S\left(h_{j-1}^{(1)}\right)\right) \\
& =|H|^{r+1} \sum_{\left(h_{i}\right)} \sum_{\left(\kappa_{i}\right)} \sum_{\left(\lambda_{i}\right)} \phi\left(\lambda_{r}^{\prime \prime \prime} \cdots \lambda_{1}^{\prime \prime \prime}\right) \phi\left(\kappa_{1}^{\prime \prime \prime} \cdots \kappa_{r}^{\prime \prime \prime}\right) \prod_{j=1}^{r} \phi\left(h_{j}^{(2)} S\left(h_{j-1}^{(1)}\right) S\left(\lambda_{j}^{\prime \prime}\right)\right) \\
& \phi\left(h_{j}^{(3)} S\left(h_{j-1}^{(4)}\right) \kappa_{j}^{\prime \prime}\right)\left|\lambda_{1}^{\prime}\right\rangle \otimes \cdots \otimes\left|\lambda_{r}^{\prime}\right\rangle \otimes \phi\left(\kappa_{1}^{\prime} ?\right) \otimes \cdots \otimes \phi\left(\kappa_{r}^{\prime} ?\right)
\end{aligned}
$$

where we used Proposition 7 in the third line.

Furthermore, using Lemma 7 we may derive

$$
\sum_{\left(\kappa_{i}\right)} \phi\left(\kappa_{1}^{\prime \prime \prime} \cdots \kappa_{r}^{\prime \prime \prime}\right) \prod_{j=1}^{r} \phi\left(b_{j} \kappa_{j}^{\prime \prime}\right) \bigotimes_{k=1}^{r} \phi\left(\kappa_{k}^{\prime} ?\right)=|H|^{-r} \sum_{\left(b_{i}\right)} \phi\left(b_{r}^{\prime \prime} \cdots b_{1}^{\prime \prime}\right) \bigotimes_{j=1}^{r} \phi\left(S\left(b_{j}^{\prime}\right) ?\right) .
$$


With this under our belt we arrive at

$$
\begin{aligned}
\widetilde{\rho}_{R}= & |H| \sum_{\left(h_{i}\right)\left(\lambda_{i}\right)} \phi\left(\lambda_{r}^{\prime \prime \prime} \cdots \lambda_{1}^{\prime \prime \prime}\right) \phi\left(h_{r}^{(4)} S\left(h_{r-1}^{(5)}\right) \cdots h_{2}^{(4)} S\left(h_{1}^{(5)}\right) h_{1}^{(4)} S\left(h_{r}^{(5)}\right)\right) \\
& \prod_{j=1}^{r} \phi\left(h_{j}^{(2)} S\left(h_{j-1}^{(1)}\right) S\left(\lambda_{j}^{\prime \prime}\right)\right)\left|\lambda_{1}^{\prime}, \ldots, \lambda_{r}^{\prime}\right\rangle \bigotimes_{k=1}^{r} \phi\left[S\left(h_{k}^{(3)} S\left(h_{k-1}^{(6)}\right)\right) ?\right] \\
= & |H| \sum_{\left(h_{i}\right)\left(\lambda_{i}\right)} \phi\left(\lambda_{r}^{\prime \prime \prime} \cdots \lambda_{1}^{\prime \prime \prime}\right) \prod_{j=1}^{r} \phi\left(h_{j}^{(2)} S\left(h_{j-1}^{(1)}\right) S\left(\lambda_{j}^{\prime \prime}\right)\right) \\
& \left|\lambda_{1}^{\prime}, \ldots, \lambda_{r}^{\prime}\right\rangle \bigotimes_{k=1}^{r} \phi\left(h_{k-1}^{(4)} S\left(h_{k}^{(3)}\right) ?\right) .
\end{aligned}
$$

Again some more preparation, namely Lemma 8 allows us to find

$$
\sum_{\left(\lambda_{i}\right)} \phi\left(\lambda_{r}^{\prime \prime \prime} \cdots \lambda_{1}^{\prime \prime \prime}\right) \prod_{j=1}^{r} \phi\left(a_{j} \lambda_{j}^{\prime \prime}\right) \bigotimes_{k=1}^{r}\left|\lambda_{k}^{\prime}\right\rangle=|H|^{-r} \sum_{\left(a_{j}\right)} \phi\left(a_{1}^{\prime \prime} \cdots a_{r}^{\prime \prime}\right) \bigotimes_{j=1}^{r}\left|S\left(a_{j}^{\prime}\right)\right\rangle
$$

and subsequently we have for the reduced density operator:

$$
\tilde{\rho}_{R}=|H|^{1-r} \sum_{\left(h_{i}\right)} \bigotimes_{j=1}^{r}\left|h_{j}^{(2)} S\left(h_{j-1}^{(1)}\right)\right\rangle \bigotimes_{k=1}^{r} \phi\left(h_{k-1}^{(4)} S\left(h_{k}^{(3)}\right) ?\right) .
$$

Finally, it can be shown that the reduced density operator of the region $R$ takes the simple form

$$
\begin{gathered}
\tilde{\rho}_{R}=|H|^{1-r} \sum_{\left(h_{i}\right)}\left|h_{1}^{(1)}\right\rangle \otimes \cdots \otimes\left|h_{r-1}^{(1)}\right\rangle \otimes\left|S\left(h_{r-1}^{(4)} \cdots h_{1}^{(4)}\right)\right\rangle \\
\otimes \phi\left(S\left(h_{1}^{(2)}\right) ?\right) \otimes \cdots \otimes \phi\left(S\left(h_{r-1}^{(2)}\right) ?\right) \otimes \phi\left(h_{r-1}^{(3)} \cdots h_{1}^{(3)} ?\right) .
\end{gathered}
$$

up to normalization. It is easy to see that

$$
\begin{aligned}
\operatorname{tr}\left(\widetilde{\rho}_{R}\right) & =|H|^{1-r} \sum_{\left(h_{i}\right)} \prod_{j=1}^{r-1} \phi\left(S\left(h_{j}^{(2)}\right) h_{j}^{(1)}\right) \phi\left(h_{r-1}^{(3)} \cdots h_{1}^{(3)} S\left(h_{r-1}^{(4)} \cdots h_{1}^{(4)}\right)\right) \\
& =|H|^{1-r}
\end{aligned}
$$

and hence we can fix the normalization by setting $N=|H|^{1-r}$.

Furthermore it is not difficult to show that $\rho_{R}$ is proportional to a projector. Indeed, consider

$$
\begin{aligned}
\rho_{R}^{2}= & \sum_{\left(h_{i}\right)\left(\lambda_{i}\right)} \prod_{j=1}^{r-1} \phi\left(S\left(h_{j}^{(2)}\right) \lambda_{j}^{(1)}\right) \phi\left(h_{r-1}^{(3)} \cdots h_{1}^{(3)} S\left(\lambda_{r-1}^{(4)} \cdots \lambda_{1}^{(4)}\right)\right) \\
& \left|h_{1}^{(1)}\right\rangle \otimes \cdots \otimes\left|h_{r-1}^{(1)}\right\rangle \otimes\left|S\left(h_{r-1}^{(4)} \cdots h_{1}^{(4)}\right)\right\rangle \\
& \otimes \phi\left(S\left(\lambda_{1}^{(2)}\right) ?\right) \otimes \cdots \otimes \phi\left(S\left(\lambda_{r-1}^{(2)}\right) ?\right) \otimes \phi\left(\lambda_{r-1}^{(3)} \cdots \lambda_{1}^{(3)} ?\right) \\
= & |H|^{1-r} \rho_{R} .
\end{aligned}
$$


This means that the spectrum of $\rho_{R}$ is flat.

Finally one can prove that the rank of the reduced density operator $\rho_{R}$ is given by $|H|^{r-1}$ hence each non-zero eigenvalue equals $|H|^{1-r}$. Then the Rényi entropies read

$$
S_{\alpha}\left(\rho_{R}\right)=\frac{1}{1-\alpha} \log \left(\operatorname{tr}\left(\rho_{R}^{\alpha}\right)\right)=|\partial R| \log |H|-\log |H| .
$$

independently of $\alpha$.

Remark 2. We would like to stress that any reduced density operator $\rho_{R}$ obtained from the states $\left|\psi_{K, N}\right\rangle$ is proportional to a projector as has been noted before for the case of group algebras at the top of the hierarchy 20]. In other words, the entanglement spectrum is flat and all Rényi entropies are equal.

While this proof yields exactly the entanglement entropy one would expect it does leave open one question: what is the mechanism of this universal correction to the area law in these Hopf tensor network states?

It turns out that one can learn the answer by analyzing the boundary configurations $\left(f_{1}, \ldots, f_{r}\right) \subset X^{r}$ of either $\left|\psi_{R}\left(f_{1}, \ldots, f_{r}\right)\right\rangle$ or $\left|\psi_{\bar{R}}\left(f_{1}, \ldots, f_{r}\right)\right\rangle$ in a particular canonical basis which is natural to the problem. Namely, by a generalized Fourier construction [10,12] one can extend the grouplike elements $\mathcal{G}(H)$ of $H$ to a linear basis the dual of which we denote $\mathcal{B} \subset X$. This dual basis is essentially unique and therefore serves as our canonical choice. For example, if $H$ is a group algebra one trivially has $\mathcal{B}=\left\{\delta_{g} \mid g \in G\right\}$.

Then the dependence of, say, the interior states $\left|\psi_{R}\left(f_{1}, \ldots, f_{r}\right)\right\rangle$ on the boundary configuration $\left(f_{1}, \ldots, f_{r}\right) \subset \mathcal{B}^{r}$ is radically different if $H$ is a group algebra, the dual of a group algebra or a non-trivial Hopf $C^{*}$-algebra like $H_{8}$. Indeed, one may show that in the first case all non-zero interior states are orthogonal and share the same norm. Furthermore an interior state with boundary configuration $\left(\delta_{g_{1}}, \ldots, \delta_{g_{r}}\right)$ vanishes precisely if $g_{1} \cdots g_{r} \neq e$. Hence the topological constraint is realized by simple group multiplication around the boundary. In the case when $H$ equals the dual of a group algebra, any interior state $\left|\psi_{R}\left(f_{1}, \ldots, f_{r}\right)\right\rangle$ is different from zero, however, altogether they cease to be orthogonal to each other. Instead, they come in bunches of exactly $|H|$ identical states each. This obviously defines an equivalence relation among different boundary configurations. It is only these equivalence classes which are orthogonal and need to be summed over if we want to turn (5.3) into a proper Schmidt decomposition. This is the second mechanism how the topological constraint is implemented in a quantum double model ground state. Finally, if $H$ is a nontrivial Hopf $C^{*}$-algebra like $H_{8}$ one will observe a combination of the two mechanisms just described.

\section{Discussion}

First of all, we have developed a tensor network language, based on the formalism of finite-dimensional Hopf $C^{*}$-algebras, which is both more flexible and more natural than conventional descriptions for topological states. We have given rules to evaluate tensor network diagrams by means of tensor traces and to construct 
quantum states on the lattice, as well as to perform operations related to the spatial lattice structure, such as cutting and joining along region boundaries.

This tensor network language has been shown to directly lead to the construction of well-known topologically ordered states, namely ground states of Kitaev's quantum double models based on groups [26. But in addition, we also obtain the extension of Kitaev's construction to Hopf algebras. This is physically relevant because the quantum double models based on Hopf algebras is the smallest class of models allowing for a non-Abelian electric-magnetic duality in topological models [12. All these states are written, in a basis-independent way, in the form of tensor networks involving the intrinsic Hopf $C^{*}$-algebra structure only.

Relaxing this latter property leads us to a hierarchy of states defined from different subalgebras of a given Hopf $C^{*}$-algebra. We study the classes obtained from group algebras, that is, Kitaev's original models, and show that the hierarchy arises from the mechanism of condensation of topological charges [4,5, and so we conjecture that this mechanism can be described in general in our language.

Furthermore the hierarchy states can be regarded as ground states of certain frustration-free Hamiltonians beyond the $\mathrm{D}(H)$-model. These are obtained from Theorem 2 by replacing the Haar integrals $h \in H$ and $\phi \in X$ with $h_{A} \in A$ and $\phi_{B} \in B$ respectively where $A \subset H$ and $B \subset X$ are Hopf $C^{*}$-subalgebras.

Not least, we have established isometric mappings defining entanglement renormalization [45,46] extending the work of [1] for the states at the top of the hierarchy. This is a systematic procedure to thin out degrees of freedom keeping the topological nature of the states, and hence their nonlocal properties, intact. Our computation of the topological entanglement entropy is an application of this general scheme.

From a more philosophical viewpoint, our graphical language brings tensor networks closer to algebraic sources; and, in particular, it highlights the role of Hopf $C^{*}$-algebras as natural spaces for transformations and symmetries of quantum many-body systems.

We also give a broader perspective of Kitaev's original quantum double construction, giving the mathematical background necessary but emphasising the physical meaning of the different elements. Hopf $C^{*}$-algebras are shown to be natural and powerful tools in the practical analysis of two-dimensional quantum problems; we hope that the applications in this paper constitute a learn-by-doing introduction to this structure for physicists.

This work opens several areas of research we are currently pursuing. First of all, it is important to study the precise form in which charge condensation appears in the Hopf quantum double models. Next, it is natural to ask whether all topological models can be cast in quantum double model form by a suitable generalisation of our tensor networks. In [12] we conjecture that the answer is yes, and that the relevant algebraic structure is the class of weak Hopf $C^{*}$ algebras; the details of the mathematical construction and the corresponding tensor network are work in progress 13 . 


\section{A. Hopf singlets}

We collect here some lemmas, with their proofs, that are used throughout the text.

Lemma 4. Let $h \in H$ the Haar integral, $a, b, c \in H$ and $f, g \in X$. Then one has

$$
\sum_{(h)} f\left(S\left(h^{\prime \prime}\right) b\right) g\left(c h^{\prime \prime \prime}\right) a h^{\prime}=\sum_{(a)(h)} f\left(S\left(h^{\prime \prime}\right) a^{\prime \prime} b\right) g\left(c S\left(a^{\prime}\right) h^{\prime \prime \prime}\right) h^{\prime} .
$$

Proof. It is clear that the statement will follow from

$$
\sum_{(h)} a h^{\prime} \otimes S\left(h^{\prime \prime}\right) \otimes h^{\prime \prime \prime}=\sum_{(a)(h)} h^{\prime} \otimes S\left(h^{\prime \prime}\right) a^{\prime \prime} \otimes S\left(a^{\prime}\right) h^{\prime \prime \prime} .
$$

Indeed, we have

$$
\begin{aligned}
\sum_{(h)} a h^{\prime} \otimes S\left(h^{\prime \prime}\right) \otimes h^{\prime \prime \prime} & =\sum_{(a)(h)} a^{\prime} h^{\prime} \otimes S\left(h^{\prime \prime}\right) \epsilon\left(a^{\prime \prime}\right) \otimes h^{\prime \prime \prime} \\
& =\sum_{(a)(h)} a^{\prime} h^{\prime} \otimes S\left(h^{\prime \prime}\right) S\left(a^{\prime \prime}\right) a^{\prime \prime \prime} \otimes h^{\prime \prime \prime} \\
& =\sum_{(a)(h)} a^{(1)} h^{\prime} \otimes S\left(a^{(2)} h^{\prime \prime}\right) a^{(4)} \otimes \epsilon\left(a^{(3)}\right) h^{\prime \prime \prime} \\
& =\sum_{(a)(h)} a^{(1)} h^{\prime} \otimes S\left(a^{(2)} h^{\prime \prime}\right) a^{(5)} \otimes S\left(a^{(4)}\right) a^{(3)} h^{\prime \prime \prime} \\
& =\sum_{(a)} \sum_{\left(a^{\prime} h\right)}\left(a^{\prime} h\right)^{\prime} \otimes S\left(\left(a^{\prime} h\right)^{\prime \prime}\right) a^{\prime \prime \prime} \otimes S\left(a^{\prime \prime}\right)\left(a^{\prime} h\right)^{\prime \prime \prime} \\
& =\sum_{(a)(h)} \epsilon\left(a^{\prime}\right) h^{\prime} \otimes S\left(h^{\prime \prime}\right) a^{\prime \prime \prime} \otimes S\left(a^{\prime \prime}\right) h^{\prime \prime \prime} \\
& =\sum_{(a)(h)} h^{\prime} \otimes S\left(h^{\prime \prime}\right) a^{\prime \prime} \otimes S\left(a^{\prime}\right) h^{\prime \prime \prime} .
\end{aligned}
$$

Lemma 5. Let $a, b \in H$ and $h \in H$ as well as $\phi \in X$ the respective Haar integrals. Then

$$
\sum_{(h)} \phi\left(a h^{\prime \prime \prime}\right) \phi\left(b S\left(h^{\prime \prime}\right)\right) h^{\prime}=|H|^{-1} \sum_{(a)} \phi\left(b a^{\prime}\right) S\left(a^{\prime \prime}\right) .
$$

Proof. Similarly to the proof of Lemma 4 one can first show that

$$
\sum_{(h)} h^{\prime} \otimes S\left(h^{\prime \prime}\right) \otimes a h^{\prime \prime \prime}=\sum_{(a)(h)} S\left(a^{\prime \prime}\right) h^{\prime} \otimes S\left(h^{\prime \prime}\right) a^{\prime} \otimes h^{\prime \prime \prime}
$$


holds for any $a \in H$. Then we use the defining property (38) of the dual (Haar) integral:

$$
\begin{aligned}
\sum_{(h)} \phi\left(a h^{\prime \prime \prime}\right) \phi\left(b S\left(h^{\prime \prime}\right)\right) h^{\prime} & =\sum_{(a)(h)} \phi\left(h^{\prime \prime \prime}\right) \phi\left(b S\left(h^{\prime \prime}\right) a^{\prime}\right) S\left(a^{\prime \prime}\right) h^{\prime} \\
& =\sum_{(a)(h)} \phi\left[b S\left(h^{\prime \prime} \phi\left(h^{\prime \prime \prime}\right)\right) a^{\prime}\right] S\left(a^{\prime \prime}\right) h^{\prime} \\
& =\sum_{(a)(h)} \phi\left(b S\left(1_{H}\right) a^{\prime}\right) S\left(a^{\prime \prime}\right) h^{\prime} \phi\left(h^{\prime \prime}\right) \\
& =\phi(h) \sum_{(a)} \phi\left(b a^{\prime}\right) S\left(a^{\prime \prime}\right) .
\end{aligned}
$$

Lemma 6. Let $a, b, c, d \in H$ and $f, g \in X$. Furthermore let $h \in H$ and $\phi \in X$ the respective Haar integrals. Then

$$
\sum_{(h)} \phi\left(a h^{\prime}\right) f\left(b S\left(h^{\prime \prime}\right) c\right) g\left(d h^{\prime \prime \prime}\right)=|H|^{-1} \sum_{(a)} f\left(b a^{\prime \prime} c\right) g\left(d S\left(a^{\prime}\right)\right) .
$$

Proof. From (204) we get

$$
\begin{aligned}
\sum_{(h)} \phi\left(a h^{\prime}\right) f\left(b S\left(h^{\prime \prime}\right) c\right) g\left(d h^{\prime \prime \prime}\right) & =\sum_{(a)(h)} \phi\left(h^{\prime}\right) f\left(b S\left(h^{\prime \prime}\right) a^{\prime \prime} c\right) g\left(d S\left(a^{\prime}\right) h^{\prime \prime \prime}\right) \\
& =\sum_{(a)(h)} f\left(b a^{\prime \prime} c\right) g\left(d S\left(a^{\prime}\right) \phi\left(h^{\prime}\right) h^{\prime \prime}\right) \\
& =\phi(h) \sum_{(a)} f\left(b a^{\prime \prime} c\right) g\left(d S\left(a^{\prime}\right)\right)
\end{aligned}
$$

which proves the claim.

Lemma 7. Let $a, b, c, d \in H$ and $h \in H$ and $\phi \in X$ the respective Haar integrals. Then

$$
\sum_{(h)} \phi\left(h^{\prime} a\right) \phi\left(b h^{\prime \prime}\right) \phi\left(c h^{\prime \prime \prime} d\right)=|H|^{-1} \sum_{(b)} \phi\left(S\left(b^{\prime}\right) a\right) \phi\left(c S\left(b^{\prime \prime}\right) d\right) .
$$

Proof. We first show that

$$
\sum_{(h)} h^{\prime} \otimes b h^{\prime \prime} \otimes h^{\prime \prime \prime}=\sum_{(b)(h)} S\left(b^{\prime}\right) h^{\prime} \otimes h^{\prime \prime} \otimes S\left(b^{\prime \prime}\right) h^{\prime \prime \prime} .
$$

Indeed, we have

$$
\begin{aligned}
\sum_{(h)} h^{\prime} \otimes b h^{\prime \prime} \otimes h^{\prime \prime \prime} & =\sum_{(b)(h)} \epsilon\left(b^{\prime}\right) h^{\prime} \otimes b^{\prime \prime} h^{\prime \prime} \otimes h^{\prime \prime \prime} \\
& =\sum_{(b)(h)} S\left(b^{\prime}\right) b^{\prime \prime} h^{\prime} \otimes b^{\prime \prime \prime} h^{\prime \prime} \otimes h^{\prime \prime \prime}
\end{aligned}
$$




$$
\begin{aligned}
= & \sum_{(b)(h)} S\left(b^{(1)}\right) b^{(2)} h^{\prime} \otimes b^{(3)} h^{\prime \prime} \otimes \epsilon\left(b^{(4)}\right) h^{\prime \prime \prime} \\
= & \sum_{(b)(h)} S\left(b^{(1)}\right) b^{(2)} h^{\prime} \otimes b^{(3)} h^{\prime \prime} \otimes S\left(b^{(5)}\right) b^{(4)} h^{\prime \prime \prime} \\
= & \sum_{(b)} \sum_{\left(b^{\prime \prime} h\right)} S\left(b^{\prime}\right)\left(b^{\prime \prime} h\right)^{\prime} \otimes\left(b^{\prime \prime} h\right)^{\prime \prime} \otimes S\left(b^{\prime \prime \prime}\right)\left(b^{\prime \prime} h\right)^{\prime \prime \prime} \\
= & \sum_{(b)(h)} S\left(b^{\prime}\right) \epsilon\left(b^{\prime \prime}\right) h^{\prime} \otimes h^{\prime \prime} \otimes S\left(b^{\prime \prime \prime}\right) h^{\prime \prime \prime} \\
= & \sum_{(b)(h)} S\left(b^{\prime}\right) h^{\prime} \otimes h^{\prime \prime} \otimes S\left(b^{\prime \prime}\right) h^{\prime \prime \prime} .
\end{aligned}
$$

Again by property (38) of the dual (Haar) integral we deduce:

$$
\begin{aligned}
\sum_{(h)} \phi\left(h^{\prime} a\right) \phi\left(b h^{\prime \prime}\right) \phi\left(c h^{\prime \prime \prime} d\right) & =\sum_{(b)(h)} \phi\left(S\left(b^{\prime}\right) h^{\prime} a\right) \phi\left(h^{\prime \prime}\right) \phi\left(c S\left(b^{\prime \prime}\right) h^{\prime \prime \prime} d\right) \\
& =\sum_{(b)(h)} \phi\left(S\left(b^{\prime}\right) a\right) \phi\left(c S\left(b^{\prime \prime}\right) \phi\left(h^{\prime}\right) h^{\prime \prime} d\right) \\
& =\phi(h) \sum_{(b)} \phi\left(S\left(b^{\prime}\right) a\right) \phi\left(c S\left(b^{\prime \prime}\right) d\right) .
\end{aligned}
$$

Lemma 8. Let $a, b, c \in H$ and $h \in H$ as well as $\phi \in X$ the respective Haar integrals. Then

$$
\sum_{(h)} \phi\left(h^{\prime \prime} a\right) \phi\left(b h^{\prime \prime \prime} c\right) h^{\prime}=|H|^{-1} \sum_{(a)} \phi\left(b S\left(a^{\prime \prime}\right) c\right) S\left(a^{\prime}\right) .
$$

Proof. Along the same lines as the proof of Lemma 7 one can show that

$$
\sum_{(h)} h^{\prime} \otimes h^{\prime \prime} a \otimes h^{\prime \prime \prime}=\sum_{(a)(h)} h^{\prime} S\left(a^{\prime}\right) \otimes h^{\prime \prime} \otimes h^{\prime \prime \prime} S\left(a^{\prime \prime}\right) .
$$

As before the rest then follows from the properties of the dual Haar integral.

Acknowledgements. We gratefully acknowledge discussions with David Pérez-García, J. Ignacio Cirac, Norbert Schuch and Sergey Bravyi. For valuable comments on an earlier version of this manuscript we would like to thank Liang Kong and Joost Slingerland. M. A. thanks Jürgen Fuchs for an illuminating introduction to Hopf algebras.

\section{References}

1. Miguel Aguado and Guifré Vidal. Entanglement renormalization and topological order. Phys. Rev. Lett., 100(7):070404, 2008.

2. Philip W. Anderson. The resonating valence bond state in $\mathrm{La}_{2} \mathrm{CuO}_{4}$ and superconductivity. Science, 235:1196, 1987.

3. Manuel Asorey. Topological phases of quantum theories. Chern-Simons theory. J. Geom. Phys., 11(1-4):63-94, 1993. 
4. F. Alexander Bais, Bernd J. Schroers, and Joost K. Slingerland. Broken quantum symmetry and confinement phases in planar physics. Phys. Rev. Lett., 89(18):181601, 2002.

5. F. Alexander Bais, Bernd J. Schroers, and Joost K. Slingerland. Hopf symmetry breaking and confinement in (2+1)-dimensional gauge theory. J. High Energ. Phys., 2003(5):068, 2003.

6. Immanuel Bloch, Jean Dalibard, and Wilhelm Zwerger. Many-body physics with ultracold gases. Rev. Mod. Phys., 80(3):885-964, 2008.

7. Sergey Bravyi and Matthew B. Hastings. A short proof of stability of topological order under local perturbations, 2010.

8. Sergey Bravyi, Matthew B. Hastings, and Spyridon Michalakis. Topological quantum order: stability under local perturbations, 2010.

9. Sergey Bravyi and Alexei Y. Kitaev. Quantum codes on a lattice with boundary, 1998

10. Oliver Buerschaper and Miguel Aguado. Mapping Kitaev's quantum double lattice models to Levin and Wen's string-net models. Phys. Rev. B, 80(15):155136, 2009.

11. Oliver Buerschaper, Miguel Aguado, and Guifré Vidal. Explicit tensor network representation for the ground states of string-net models. Phys. Rev. B, 79(8):085119, 2009.

12. Oliver Buerschaper, Matthias Christandl, Liang Kong, and Miguel Aguado. Electricmagnetic duality and topological order on the lattice, 2010.

13. Oliver Buerschaper and et al. In preparation.

14. Xie Chen, Bei Zeng, Zheng-Cheng Gu, Isaac L. Chuang, and Xiao-Gang Wen. Tensor product representation of topological ordered phase: necessary symmetry conditions, 2010.

15. Eric Dennis, Alexei Y. Kitaev, Andrew Landahl, and John Preskill. Topological quantum memory. J. Math. Phys., 43(9):4452, 2002.

16. Vladimir G. Drinfeld. Quantum groups. In Andrew M. Gleason, editor, Proceedings of the International Congress of Mathematicians (Berkeley, 1986), pages 798-820. Amer. Math. Soc., 1987.

17. Jens Eisert, Marcus Cramer, and Martin B. Plenio. Area laws for the entanglement entropy. Rev. Mod. Phys., 82:277-306, 2010.

18. Pavel Etingof and Shlomo Gelaki. Semisimple Hopf algebras of dimension $p q$ are trivial. J. Alg., 210(2):664-669, 1998.

19. Mark Fannes, Bruno Nachtergaele, and Reinhard F. Werner. Finitely correlated states on quantum spin chains. Commun. Math. Phys., 144(3):443-490, 1992.

20. Steven T. Flammia, Alioscia Hamma, Taylor L. Hughes, and Xiao-Gang Wen. Topological entanglement Rényi entropy and reduced density matrix structure. Phys. Rev. Lett., 103(26):261601, 2009.

21. Michael H. Freedman, Alexei Y. Kitaev, and Zhenghan Wang. Simulation of topological field theories by quantum computers. Commun. Math. Phys., 227(3):587-603, 2002.

22. Michael H. Freedman, Michael J. Larsen, and Zhenghan Wang. A modular functor which is universal for quantum computation. Commun. Math. Phys., 227(3):605-622, 2002

23. Zheng-Cheng Gu, Michael A. Levin, Brian Swingle, and Xiao-Gang Wen. Tensor-product representations for string-net condensed states. Phys. Rev. B, 79(8):085118, 2009.

24. G. I. Kac and V. G. Paljutkin. Finite ring groups. Trans. Moscow Math. Soc., 1966:251294,1966

25. Christian Kassel. Quantum groups. Springer, Berlin, 2nd edition, 1995.

26. Alexei Y. Kitaev. Fault-tolerant quantum computation by anyons. Ann. Phys., 303(1):230,2003

27. Alexei Y. Kitaev and John Preskill. Topological entanglement entropy. Phys. Rev. Lett. 96(11):110404, 2006

28. Anatoli Klimyk and Konrad Schmüdgen. Quantum groups and their representations. Springer, Berlin, 1997.

29. Vijay Kodiyalam, Zeph A. Landau, and V. S. Sunder. The planar algebra associated to a Kac algebra. Proceedings of the Indian Academy of Science, 113(1):15-51, 2003.

30. Robert König, Ben W. Reichardt, and Guifré Vidal. Exact entanglement renormalization for string-net models. Phys. Rev. B, 79(19):195123, 2009.

31. Michael A. Levin and Xiao-Gang Wen. String-net condensation: a physical mechanism for topological phases. Phys. Rev. B, 71(4):045110, 2005.

32. Michael A. Levin and Xiao-Gang Wen. Detecting topological order in a ground state wave function. Phys. Rev. Lett., 96(11):110405, 2006.

33. Akira Masuoka. Semisimple Hopf algebras of dimension 6, 8. Israel J. Math., 92(1-3):361$373,1995$.

34. Akira Masuoka. The $p^{n}$ theorem for semisimple Hopf algebras. Proc. Amer. Math. Soc. 124(3):735-737, 1996. 
35. Carlos Mochon. Anyon computers with smaller groups. Phys. Rev. A, 69(3):032306, 2004.

36. Valentin Murg, Frank Verstraete, and J. Ignacio Cirac. Variational study of hard-core bosons in a two-dimensional optical lattice using projected entangled pair states. Phys. Rev. A, 75(3):033605, 2007.

37. Florian Nill and Kornél Szlachányi. Quantum chains of Hopf algebras with quantum double cosymmetry. Commun. Math. Phys., 187(1):159-200, 1997.

38. Tomotoshi Nishino. Density matrix renormalization group method for 2D classical models. J. Phys. Soc. Jpn., 64(10):3598-3601, 1995.

39. David Pérez-García, Frank Verstraete, Michael M. Wolf, and J. Ignacio Cirac. PEPS as unique ground states of local Hamiltonians. Quant. Inf. Comp., 8(6\&7):0650, 2008.

40. Hans-Jürgen Schneider. Lectures on Hopf algebras. Trabajos de Matemática, 1995:31, 1995.

41. Norbert Schuch, J. Ignacio Cirac, and David Pérez-García. PEPS as ground states: degeneracy and topology, 2010.

42. Frank Verstraete and J. Ignacio Cirac. Renormalization algorithms for quantum-many body systems in two and higher dimensions, 2004.

43. Frank Verstraete and J. Ignacio Cirac. Valence-bond states for quantum computation. Phys. Rev. A, 70(6):060302(R), 2004.

44. Frank Verstraete, Michael M. Wolf, David Pérez-García, and J. Ignacio Cirac. Criticality, the area law, and the computational power of projected entangled pair states. Phys. Rev. Lett., 96(22):220601, 2006.

45. Guifré Vidal. Entanglement renormalization. Phys. Rev. Lett., 99(22):220405, 2007.

46. Guifré Vidal. Class of quantum many-body states that can be efficiently simulated. Phys. Rev. Lett., 101(11):110501, 2008.

47. Guifré Vidal, José Ignacio Latorre, Enrique Rico Ortega, and Alexei Y. Kitaev. Entanglement in quantum critical phenomena. Phys. Rev. Lett., 90(22):227902, 2003.

48. Xiao-Gang Wen. Topological orders and edge excitations in fractional quantum Hall states. Adv. Phys., 44(5):405-473, 1995.

49. Edward Witten. Topological quantum field theory. Commun. Math. Phys., 117(3):353386,1988

Communicated by Unknown 